Detailed studies of the Meshaheed gold prospect, Kingdom of Saudi Arabia by

\author{
Charles w. Smith
}

Open-File Report 86-30

Report prepared for Ministry of Petroleum and Mineral Resources, Deputy Ministry for Mineral Resources, Saudi Arabia

This report is preliminary and has not been reviewed for conformity with U.S. Geological Survey editorial standards and stratigraphic nomenclature.

I/ U.S. Geological Survey (retired) 


\section{CONTENTS}

Page

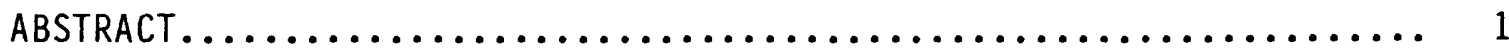

INTRODUCTION.................................... 2

ACKNOWLEDGEMENTS ............................... 4

GEOLOGY................................... 5

ECONOMIC GEOLOGY................................. 9

Molybdenite-bearing quartz stockworks.................. 9

Gold-stibnite-quartz veins........................ 10

Diorite dike.................................... 10

Hydrothermally altered shear zones..................... 10

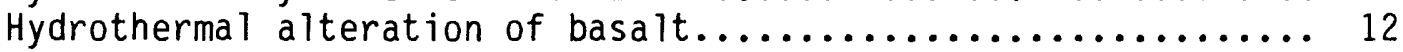

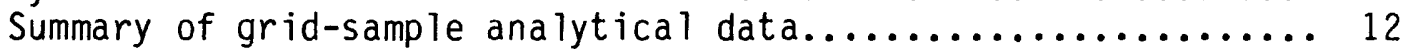

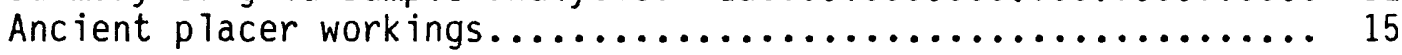

TRENCHING IN BEDROCK............................ 16

GROUND FOLLOW-UP STUDIES OF GEOPHYSICAL ANOMALIES ............ 18

CONCLUSIONS AND RECOMMENDATIONS...................... 19

DATA STORAGE................................. 43

REFERENCES ................................... 43

\section{ILLUSTRATIONS}

Figure 1. Index map showing the location of the Meshaheed

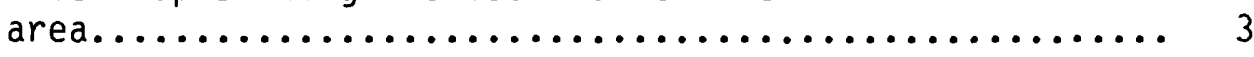

2. Geologic map of the Meshaheed area............... 6

3. Geologic map of Quartz Hill No. $1 \ldots \ldots \ldots \ldots \ldots \ldots \ldots \ldots \ldots$

4. Sample location map of the Meshaheed area........... 13

5. Geochemical map of the Meshaheed area.............. 14

\section{TABLES}

Table 1. Analytical data for rock chip sampling in the Meshaheed area............................ 20

2. Atomic absorption analytical data for trench sampling in the Meshaheed area. 


\title{
DETAILED STUDIES OF THE MESHAHEED \\ GOLD PROSPECT, \\ KINGDOM OF SAUDI ARABIA
}

by

Charles W. Smith

\begin{abstract}
The Meshaheed prospect, northeastern Arabian Shield, is in the Hadiyah formation metasediments and metabasalt that were folded, faulted, and subsequently intruded by small diorite-granodiorite plutons. Cooling and crystallization of one small granodiorite pluton caused the exsolution of a separate phase that formed a stockwork containing minor molybdenite. Gold, stibnite, and quartz were later deposited in northeast-striking faults. Large volumes of metasediments and some basalts were hydrothermally altered.

Gold is essentially confined to stibnite-quartz veins, or to zones along diorite-metasediment contacts. A large, northeast-striking, hydrothermallyaltered shear zone, was found to contain insignificant gold and accessory elements common to gold deposits in this region.

Placer-type ancient workings adjacent to a basalt ridge, small ancient workings in the basalt, and extensive hydrothermal alteration suggest that gold may have been deposited in the rock. However, sampling at these localities failed to detect significant amounts of gold, and it is concluded that ancient miners were inclined to work placer gravels low in gold content, and that the small workings in basalt are probably prospect pits.
\end{abstract}




\section{INTRODUCTION}

This report covers follow-up studies recommended as a result of previous investigations in the Meshaheed area (Smith and Samater, 1984) that included reconnaissance geologic mapping, and detailed mapping and sampling of ancient mines. An area approximately 10 by 18 kilometers northeast of Jabal Qutn was mapped by reconnaissance methods, but a smaller area about 3 by $5 \mathrm{~km}$ proved to contain several ancient mining areas and was studied in greater detail. The present study area, known as Meshaheed (MODS 01266) is located at lat $26^{\circ} 09^{\prime} 32^{\prime \prime}$ N., long $42^{\circ} 25^{\prime} 26^{\prime \prime}$ E. (fig. 1), or about $14 \mathrm{~km}$ nurtheast of Jabal Qutn in an area of low, sharp hills. Field work by the author was completed during a two-week period in March, 1984.

During the previous studies, it was noted that part of the Meshaheed area included numerous diorite cupolas, gold-bearing quartz veins, quartz stockworks containing molybdenite, and locally intensively, hydrothermally altered areas containing gold. A concurrent, regional wadi-sediment sampling survey (Allen and others, 1984), indicated that the Meshaheed area was also anomalous in copper, lead, boron, and tin. Furthermore, ground geophysical studies using audio-magnetotelluric (AMT) and telluric-electric (TE) methods (Flanigan and Zablocki, 1984) had delineated a broad, deep anomaly that coincided a hydrothermally altered area. From these observations, it was concluded that follow-up studies of hydrothermally altered areas were required. It was known that they contain gold, but grades and extent of the mineralization could not be estimated because of the reconnaissance nature of the studies and the existence of only limited outcrops. In addition, more details of the geology of the area were needed in order to better understand the genesis of the gold and molybdenite deposition.

In the present study, geologic mapping and geochemical sampling were done by use of a grid system covering an area of approximately $1 \mathrm{~km}^{2}$. North-oriented sampling-mapping lines were set at $100 \mathrm{~m}$ intervals with rock-chip sample points every $50 \mathrm{~m}$ along the lines. Two-hundred and eighty-three rock chip samples were collected, and geology observed along the lines. In addition, detailed mapping was carried out at Quartz Hill No. 1, and several samples were collected in and near small ancient workings in basalt. Also, brief follow-up studies were made of a telluric-electric anomaly located about $1 \mathrm{~km}$ east of the study area.

Four backhoe trenches were dug and sampled in an altered area, three were dug in ancient placer areas and checked for alluvial gold, and four were dug and sampled in the Pluton Southeast ancient workings located $2 \mathrm{~km}$ west of the study area (Smith and Samater, 1984). Waiss Issa Asumali was responsible for backhoe operations during trenching. 


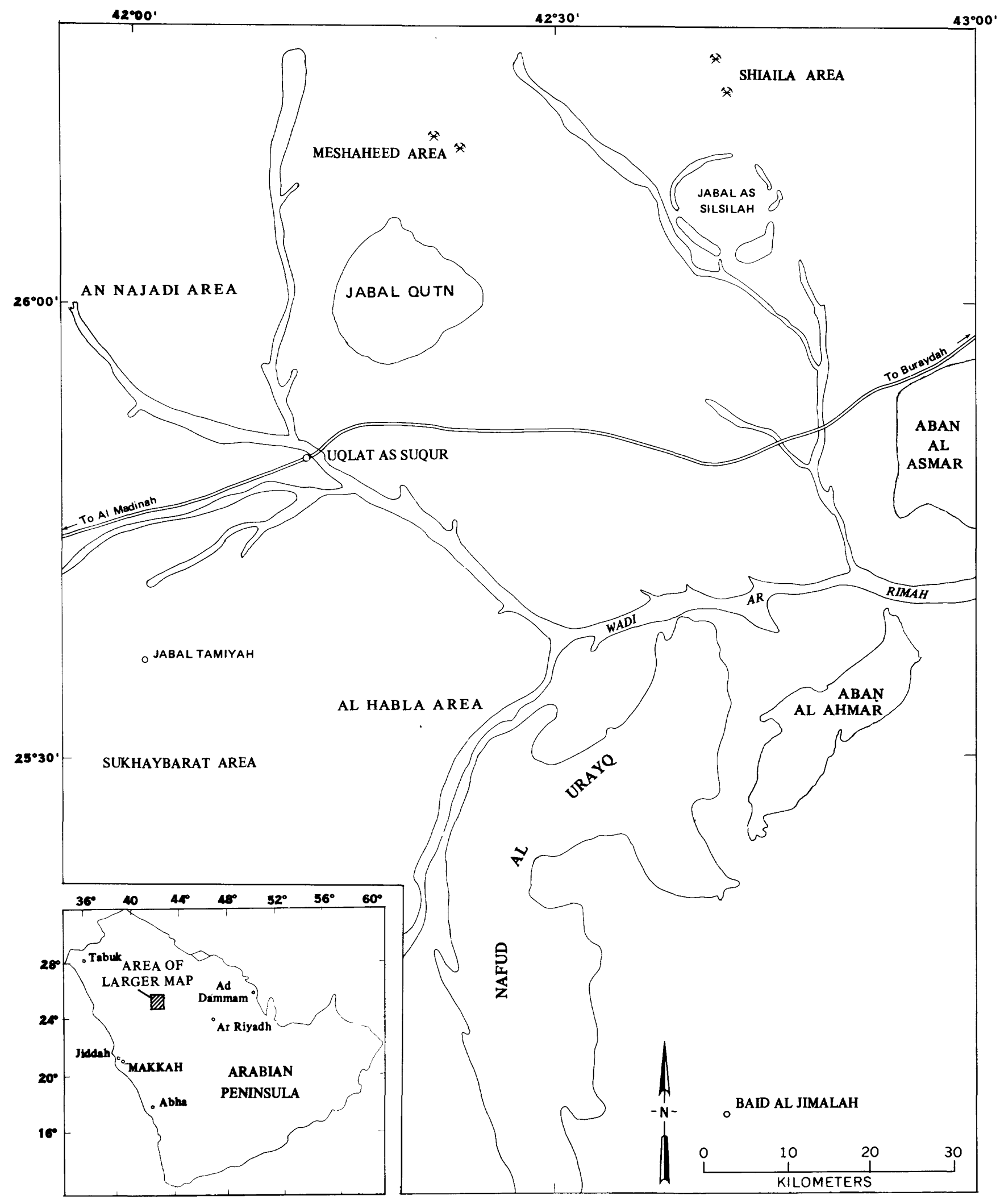

Figure 1.--Index map showing the location of the Meshaheed area 


\section{ACKNOWLEDGEMENTS}

The author is indebted to W. H. White and R. J. Kamilli, visitors to the area. White made pertinent observations concerning the molybdenite in quartz stockworks at Quartz Hill No. 1, and Kamilli found pillow basalts in the area. Both of these observations helped the author in determining geological relationships. P. L. Williams mapped the geology of the Samirah quadrangle, where the Meshaheed deposits are located, and established the geologic framework for the area.

Samples were assayed in the Jeddah laboratories of the Deputy Ministry for Mineral Resources under the direction of $K$. J. Curry. Gold and silver were assayed by atomic absorption methods, and twenty-nine other elements were assayed by semi-quantitative spectrographic methods. Thin sections and polished thin sections were made in the USGS laboratories, Jeddah, under the direction of Carl Thornber. All assistance is gratefully acknowledged.

These studies were made in accordance with an agreement between the Ministry of Petroleum and Mineral Resources and the USGS, specifically according to the work plan of the Deputy Ministry for Mineral Resources for 1403/04, sub-project 3.01.35, entitled "Assessment for gold-Meshaheed area". 


\section{GEOLOGY}

The area studied, $1 \mathrm{~km}^{2}$, encompasses Quartz Hill No. 1 and a zone south that includes metabasaltic ridges, (fig. 2). For background information concerning a much larger area that includes this study area, the reader is referred to Smith and Samater, 1984.

The geology of this area is complex, and observation is difficult because a large part of the zone is covered by alluvium and talus. Nevertheless, important geological features have been recognized by detailed mapping, such as the interlayering of metabasalt with metasediments, with only a minor part of the basalt being intrusive, contrary to our previous reconnaissance interpretation.

Metasedimentary rocks in the area have been assigned to the Hadiyah formation of the Murdama group (Williams, 1983). The Hadiyah formation rocks within the map area are mainly fine calcareous sandstones with lesser limestone, siltstone, and fine graywacke. These rocks are uniformly dark, brown-gray, calcareous, and contain no marker beds. Near plutonic contacts they are hornfelsic and are dense, fine grained, dark gray to black, and in outcrop are difficult to distinguish from basalt. In some areas near plutonic contacts, the metasediments have been granitized, some outcrops show evidence of progressive granitization ranging from long amphibole needles in sandstone to amphibole-plagioclase crystalline rock. Layering is generally not apparent, and in most of the area the sediments contain finely disseminated pyrite.

Metabasalt, locally a feldspar porphyry, is mostly interlayered with metasediments, is normally dark green to black, and contains 2 to 10 per cent pyrrhotite that is mostly disseminated, but locally occurs as crystallized masses as much as $3 \mathrm{~cm}$ in diameter. Younger, black, fine-grained basalt is intrusive as dikes and apophyses. Much of the rock is carbonatized and cut by thin quartz-calcite veinlets. Basalt pillows are well exposed locally on Basalt Hill (fig. 2), and other, more doubtful pillow structures were noted in the area.

Both metasediments and metabacalt are intruded by small diorite plutons. The diorite is medium-grained, consisting of plagioclase, hornblende, biotite, minor quartz, and in places is hydrothermally altered, mainly to kaolin. There are probably several rock types of differing ages ranging from granodiorite to melanocratic diorite in small, geologically complex areas south and east of Quartz Hill No. 1 (fig. 2), but outcrops are limited and areas were mapped according to the dominant rock type. All of the dioritic intrusions at Meshaheed are probably coeval with a great number of similar diorite-granodiorite plutons in the northeastern Arabian Shield (Cole and Hedge, 1985) that are radiometrically dated at $620 \mathrm{Ma}$ to $615 \mathrm{Ma}$. They are thought to be genetically related to gold deposits in the region (Cole, press; Smith and others, 1984; Smith and Samater; 1984).

Small plutons and dikes of granodiorite range from homogranular to porphyritic. They intrude diorite and older rocks in the central part of the map area (fig. 2), and are light brown to tan in outcrop. In places the rock is slightly sericitized and bears disseminated pyrite. A large diorite dike, intruded into mostly metasedimentary rocks, crosses the map area. A few aplite dikes that represent the last igneous phase in the area are not shown on figure 2. 


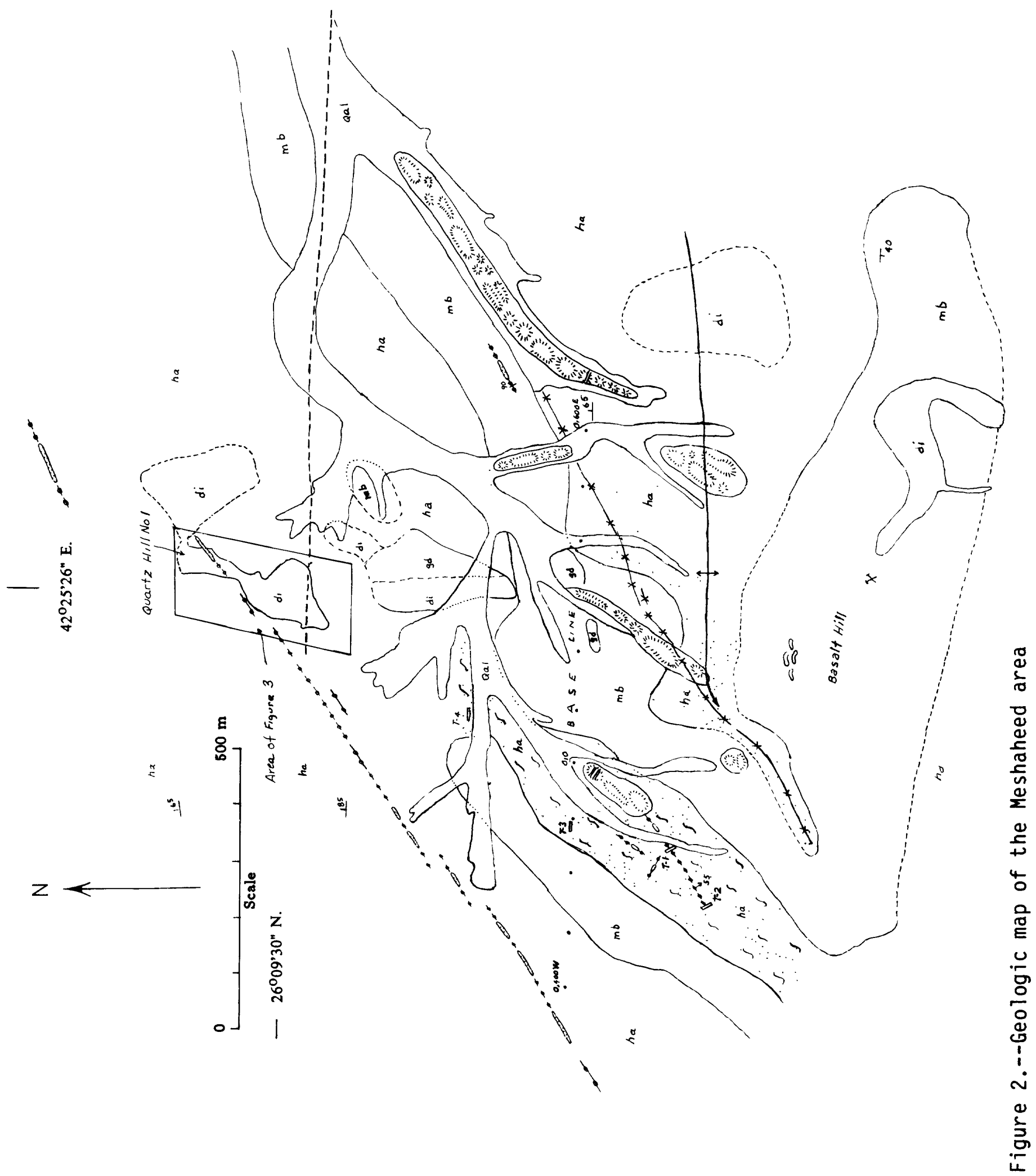



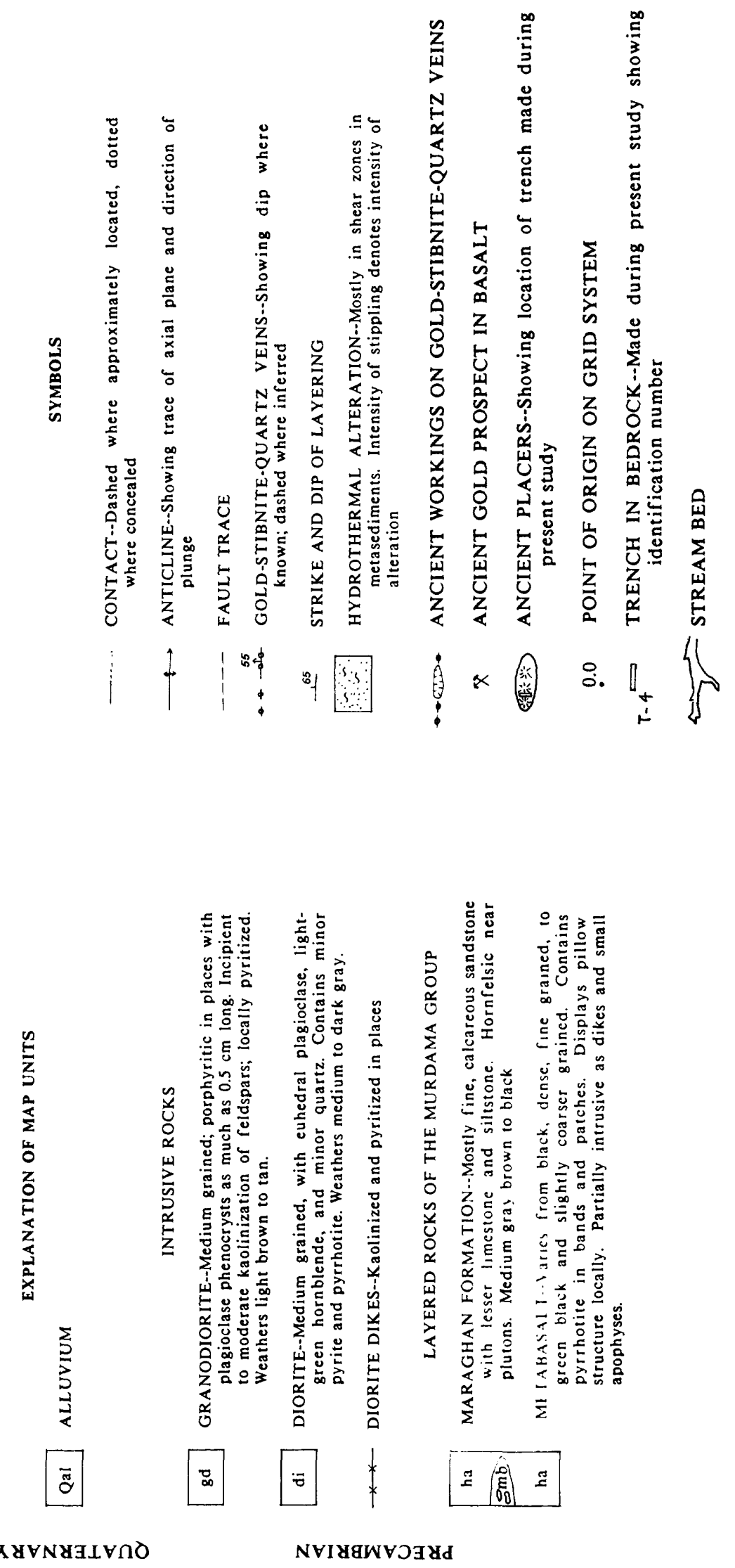

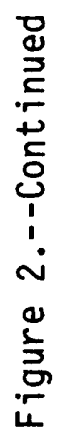


Broad, open folds and major faults in the metasedimentary rocks trend northeast and east (Williams, 1984). Regionally, northeast-trending faults are slightly offset by east-trending faults (Smith and Samater, 1984). The map area (fig. 2) indicates the same style of folding and faulting. Within this zone basalt and metasediments are folded to form what is interpreted to be a westerly plunging anticline. This interpretation is based on the drape effect of pillow basalts, layering in basalts, and strike and dip of underlying metasediments. A small diorite pluton is intruded into the axial plane of the fold. The northwestern synclinal limb of the structure has evidently been sheared along the major northeast trending shear zone and intruded by diorite, because a small, isolated basalt segment was mapped in the adjacent southeast area of Quartz Hill No. 1. A major east-trending fault also cuts this part of the fold limb, and the juncture of this fault with a northeast-trending fault served as a control for the intrusion of a small granodiorite pluton at Quartz Hill No. 1.

Williams (1984) has mapped pillow basalts about $5.5 \mathrm{~km}$ southeast of Meshaheed, where they are steeply dipping, moderately folded, and interlayered with Hadiyah formation metasediments. The two basalt localities are probably due to two independent extrusive centers because rock displacement has not occurred along northwest-southeast directions in the Meshaheed region. The Meshaheed area seems to have acted as a structurally controlled center where basalt and dioritic magmatism proliferated. 


\section{ECONOMIC GEOLOGY}

Two periods of mineral deposition are recognized at Meshaheed; the first is represented by a quartz stockwork containing minor molybdenite at Quartz Hill No. 1, and the second is represented by gold-stibnite-quartz veins in northeaststriking faults.

\section{MOLYBDENITE-BEARING QUARTZ STOCKWORKS}

A molybdenite-bearing quartz stockwork cuts moderately kaolinized granodiorite on Quartz Hill No. 1, that is partially covered with quartz lag (figs. 2 and 3). The small granodiorite pluton is cut by a quartz stockwork over much of the outcrop area. Veins and veinlets in the stockwork system range in thickness from less than $1 \mathrm{~cm}$ to nearly $50 \mathrm{~cm}$, and because of cross-cutting features, some thicker veins appear to have resulted from the final phase of quartz deposition. Stockworks are distributed unevenly throughout the intrusive rock and appear to be concentrated mainly in the areas occupied by the thicker veins. Figure 3 shows the position of the larger veins, but the areal distribution of the total stockwork zones was not mapped. Molybdenite is distributed very sparsely throughout the stockwork veins and veinlets.

Crenulate quartz layers are also common on Quartz Hill No. 1, especially along the periphery of an area of massive quartz. This type of quartz also occurs north and south of the quartz mass (fig. 3). The layered form of the quartz suggests successive episodes of inward quartz growth during formation.

White and others (1981) have described similar crenulate quartz at the Climax and Henderson molybdenum mines in Colorado, U. S. A. The quartz at these mines is associated with alkali- rich granites, forms in the vicinity of pegmatitic rocks, and in places grades into pegmatitic quartz and feldspar. The crenulate form is believed to be the result of plastic deformation. Rhythmic layering of quartz interlayered with porphyritic rock at Henderson was apparently caused by recurring increase and decrease of pressure acting on water-saturated melts containing fluorine. According to Shannon and others (1982), because the crenulate quartz is common near the outer contacts of intrusive masses, exposures of the small granodiorite pluton at Meshaheed may represent its apex.

Mutschler and others (1981) have classified 30 molybdenite stockwork deposits in the western United States as associated with either granodiorite or granite. According to White and others (1981), the granodiorite (quartz monzonite)-type deposits grade between 0.1 and $0.2 \% \mathrm{MoS}_{2}$ as opposed to grades of 0.3 to $0.45 \%$ for granite-type systems.

Both stockwork and crenulate quartz at Meshaheed contain minor molybdenite in flakes as much as $3 \mathrm{~mm}$ in diameter, or as fine crystalline material that causes the quartz to appear spotted blue-gray. Twenty-six samples of the quartz collected in previous studies gave values generally less than 100 parts per million molybdenum (Smith and Samater, 1984).

Figures 2 and 3 show the long, northeast-striking, gold-stibnite-quartz vein system intersecting the north end of the granodiorite pluton with quartz stockwork, which indicates that gold and stibnite were deposited later than the molybdenite. Previous detailed sampling of the the altered granodiorite, quartz stockwork, and gold-stibnite-quartz veins found significant gold only in the northeast-striking quartz veins, or in altered granodiorite on their projection. 
Gold-stibnite-quartz veins, the largest of which was examined and sampled in previous studies, show that gold, stibnite, and minor chalcopyrite and silver were deposited along with quartz in northeast-striking faults at Meshaheed. Most of the quartz is also rich in arsenic, but arsenic-bearing minerals have not been observed. The longer, northernmost vein system containing numerous ancient workings intersects Quartz Hill No. 1 (figs. 2 and 3), and higher gold grades were obtained from quartz dump sampling adjacent to the small pluton. Veins in this system are probably lenticular and less than $1 \mathrm{~m}$ thick.

A shorter and narrower northeast-striking gold-stibnite-quartz vein is in sheared and intensely hydrothermally altered metasedimentary rocks south of the base line at about 100 west (fig. 2). The vein, exposed by trenching in this study, is approximately $10 \mathrm{~cm}$ thick, dips 55 degrees south, in places is composed mainly of stibnite, and assays moderately high in gold. The length of the vein is at least $175 \mathrm{~m}$ according to exposures in trenches and one ancient pit. Another northeast-striking vein consisting of quartz with moderate stibnite is exposed in an ancient workings in basalt about $200 \mathrm{~m}$ northeast of point 600 east on the base line. The vein exposed in the workings is $30 \mathrm{~cm}$ thick, dips vertically, and quartz on dumps contains stibnite. The strike length of this vein could not be determined because of cover, but it is probably a short lens. Two assays, one of vein material and one of altered wall rock, are 10.6 and $3.6 \mathrm{ppm}$ gold, respectively.

\section{DIORITE DIKE}

A northeast-striking diorite dike intrudes mainly metasediments in the study area (fig. 2). The dike is fine to medium grained and light gray, and mafic minerals have been almost completely altered to chlorite. The gold contents of two samples from two localities of moderately hydrothermally altered sedimentary rocks in selvages of the dike are 0.4 and $8.0 \mathrm{ppm}$ gold.

\section{HYDROTHERMALLY ALTERED SHEAR ZONES}

A northeast-striking, hydrothermally altered shear zone crosses the base line at points 0.0 and 100 west, is $150 \mathrm{~m}$ wide, extends at least $800 \mathrm{~m}$, and disappears beneath alluvium on the northeast end (fig. 2). It contains the thin gold-stibnite-quartz vein previously described. Metasedimentary rocks within the zone are altered to quartz, sericite, pyrite, kaolin, and carbonate. Original rock textures are completely obliterated by the alteration, and types of alteration vary throughout the zone. Some outcrops show completely silicified and pyritized rock, whereas others are intensely kaolinized. Much of the rock is a quartz-sericite-pyrite-carbonate cut by thin quartz-calcite stringers. Hematite in bands as much as $30 \mathrm{~cm}$ thick and manganese oxide associated with siliceous zones are prevalent.

Metasedimentary rocks are similarly hydrothermally altered in the crest of the plunging anticline south and adjacent to the larger altered zone (fig. 2). This area may also be sheared, but definite indications of shearing were not noted. 


\section{EXPLANATION OF MAP UNITS}

gd

ha

GRANODIORITE--Moderately kaolinized. Cut by quartz stockwork containing sparse molybdenite

MARAGHAN FORMATION--Mostly fine, calcareous sandstone. Hornfelsic and partially granitized in places

\section{SYMBOLS}

CONTACT--Dotted where concealed

$\uparrow^{60}$

QUARTZ VEIN--Milky white, showing dip where known

6750

QUARTZ MASS--Showing crenulated banding and dip of walls Half circles show direction of crystal growth

QUARTZ LAYER--Showing crenulated banding. Half circles show direction of crystal.growth

ANCIENT WORKINGS ON GOLD-STIBNITE-QUARTZ

VEINS--Showing trace of inferred vein

0

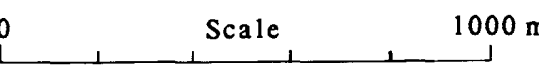

ha

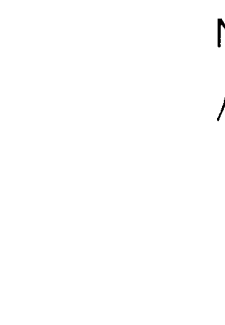

$\mathrm{N}$
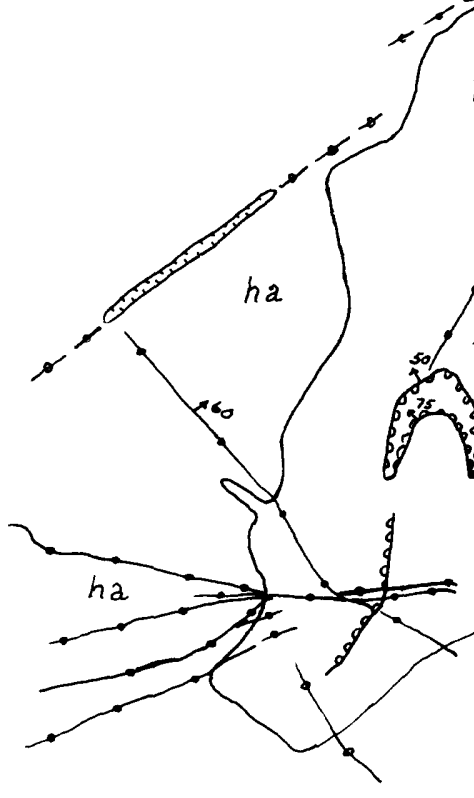
1

QUARTZ HILL NO. 1

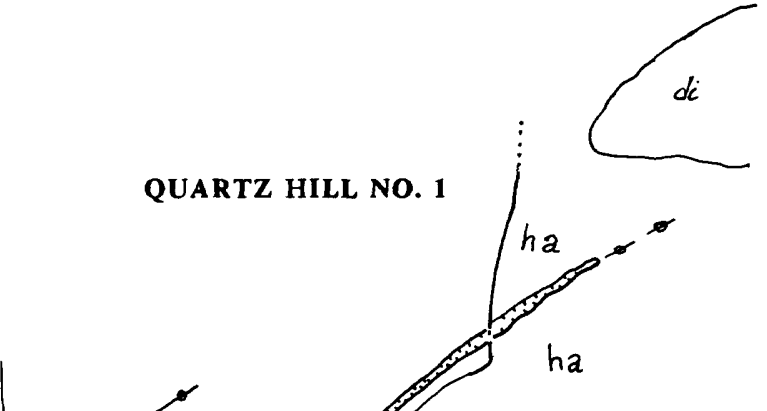


Areas of hydrothermal alteration in basalt are more difficult to define, but it appears that the rock is altered over large areas. Thin sections show fine fractures occupied by quartz, calcite, and pyrrhotite. Carbonatization is the prevalent type of hydrothermal alteration. In places, the rock is locally altered to carbonate around quartz-calcite veinlets, and some rocks are locally carbonatized giving a spotted texture. Other areas show rock totally affected by carbonatization, and in some zones accompanied by pyrrhotite. Precious metals were not detected by sampling in these zones. However, a few spurious high values in tungsten, arsenic, and boron were obtained (fig. 5). A small diorite pluton intrudes basalt in the southern part of the map area (fig. 2), and small ancient workings, probably no more than prospects, are located near its western end. Diorite is not present in the immediate area of the workings, but it is believed that the pluton and accompanying hydrothermal fluids are responsible for the carbonatization and quartz-calcite veinlets in the area. Sampling in the workings area found no gold or silver, and only one sample contained detectable arsenic $(700 \mathrm{ppm})$. Gold deposition may have been very localized and consequently missed by our sampling. There is no doubt that gold is present in the basalt because ancient miners expended a great amount of effort recovering the metal from talus slopes eroding from Basalt Hill.

\section{SUMMARY OF GRID-SAMPLE ANALYTICAL DATA}

The primary purpose of the rock chip sampling and geological mapping program was to define, if possible, larger areas of gold mineralization. Hydrothermally altered areas, such as the northeast-striking shear zone in metasediments, carbonatized zones in basalt, and contacts around diorite plutons were believed to have some potential, since previous reconnaissance sampling had found low gold values in some of these rocks. Also, since reconnaissance wadi-sediment sampling had defined Meshaheed as the center of an anomalous zone in lead, copper, boron, and tin (Allen and others, 1984), rock chip sampling by use of a grid system was considered to be a good test of the area. Atomic absorption analyses for gold and silver, and spectrographic analyses for twenty-nine other elements for all of the grid sampling are given in table 1. Sample locations with numbers are shown on figure 4 . Plots of gold values $0.2 \mathrm{ppm}$, or higher; silver values $1.0 \mathrm{ppm}$, or higher; and notable values of other elements are shown on figure 5. Statistical analyses of the chemical data were not used because most of the critical elements such as $\mathrm{Au}, \mathrm{Ag}, \mathrm{Mo}, \mathrm{As}, \mathrm{Sb}, \mathrm{W}$, and $\mathrm{Bi}$ are in amounts below the detection limits of the analytical methods (atomic absorption with partial digestion for gold and silver, and semi-quantitative spectrographic methods for the remaining elements) in most samples.

Most of the grid sampling detected no gold, and of the few samples in which gold was detected, only two contain more than one part per million (ppm) of that metal. One of these samples (200839) is of hydrothermally altered dump rock from an ancient working on the principal northeast-striking vein system in the area. The other (200772) is of pyritized (metasedimentary ?) rock in the selvage of the large northeast-striking diorite dike that crosses the map area (figs. 2 and 5 ). Three additional samples containing less than 1 ppm gold are from points near, or on the projection of the principal northeast-striking vein system. Several samples from hydrothermally altered shear zones contain less than 1 ppm gold; otherwise, samples within these zones are gold-free. Two samples from the basalt lens northwest and adjacent to the larger hydrothermally altered zone contain less than $1 \mathrm{ppm}$ gold. This basalt lens is also hydrothermally altered. 


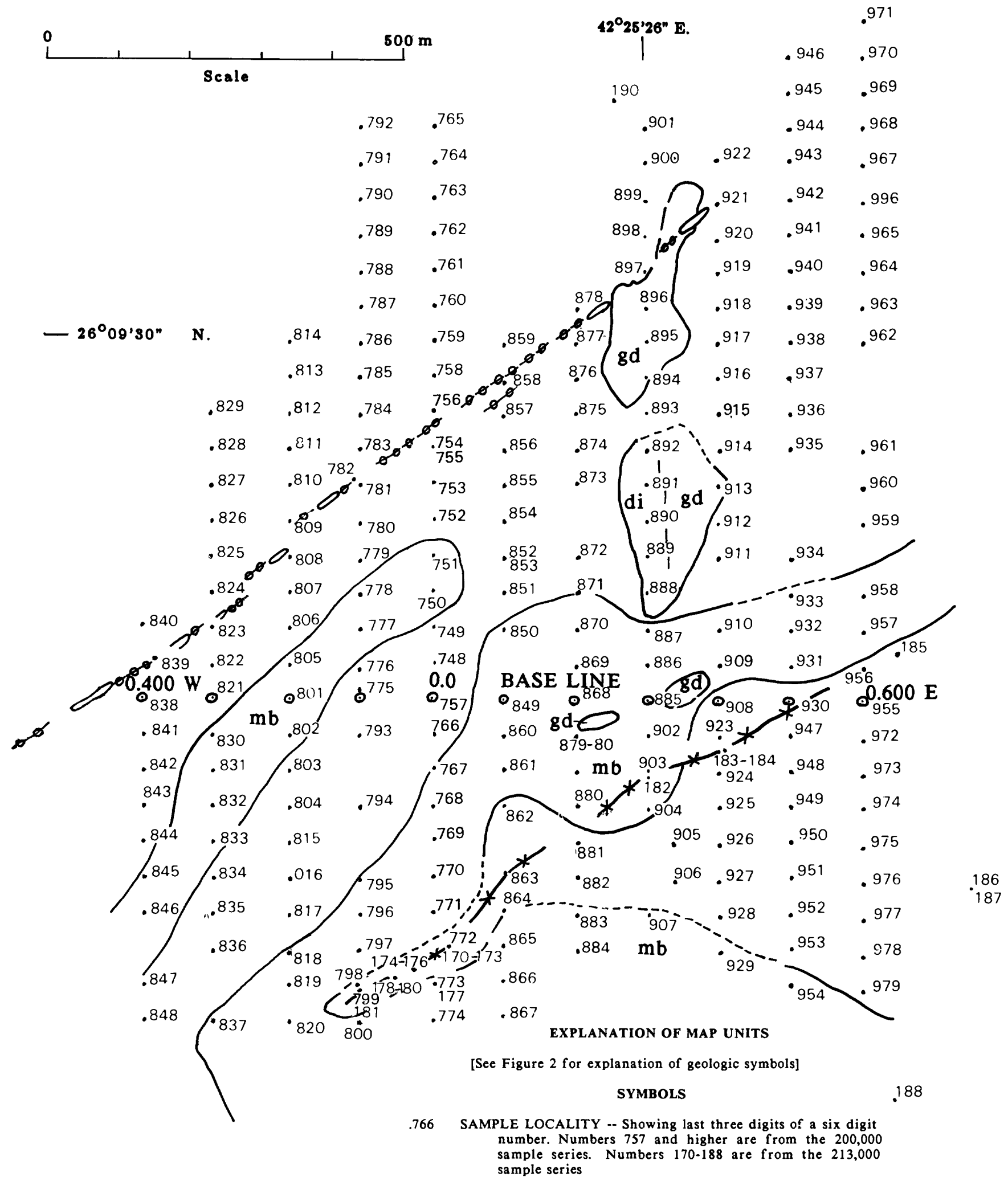

Figure 4.--Sample locality map of the Meshaheed area 
EXPLANATION OF MAP UNITS

[See Figure 2 for explanation of geologic symbols]

SYMBOLS

$42^{\circ} 25^{\prime} 26^{\prime \prime} \mathrm{E}$.

Au -0.5

As-500

Rock chip sample locality showing anomalous values $0.5 \mathrm{ppm}$ gold and $500 \mathrm{ppm}$ arsenic

W. 200

- Rock chip sample locality containing no significant elements

Gold values less than $0.2 \mathrm{ppm}$ and silver values less than 1.0 ppm not plotted

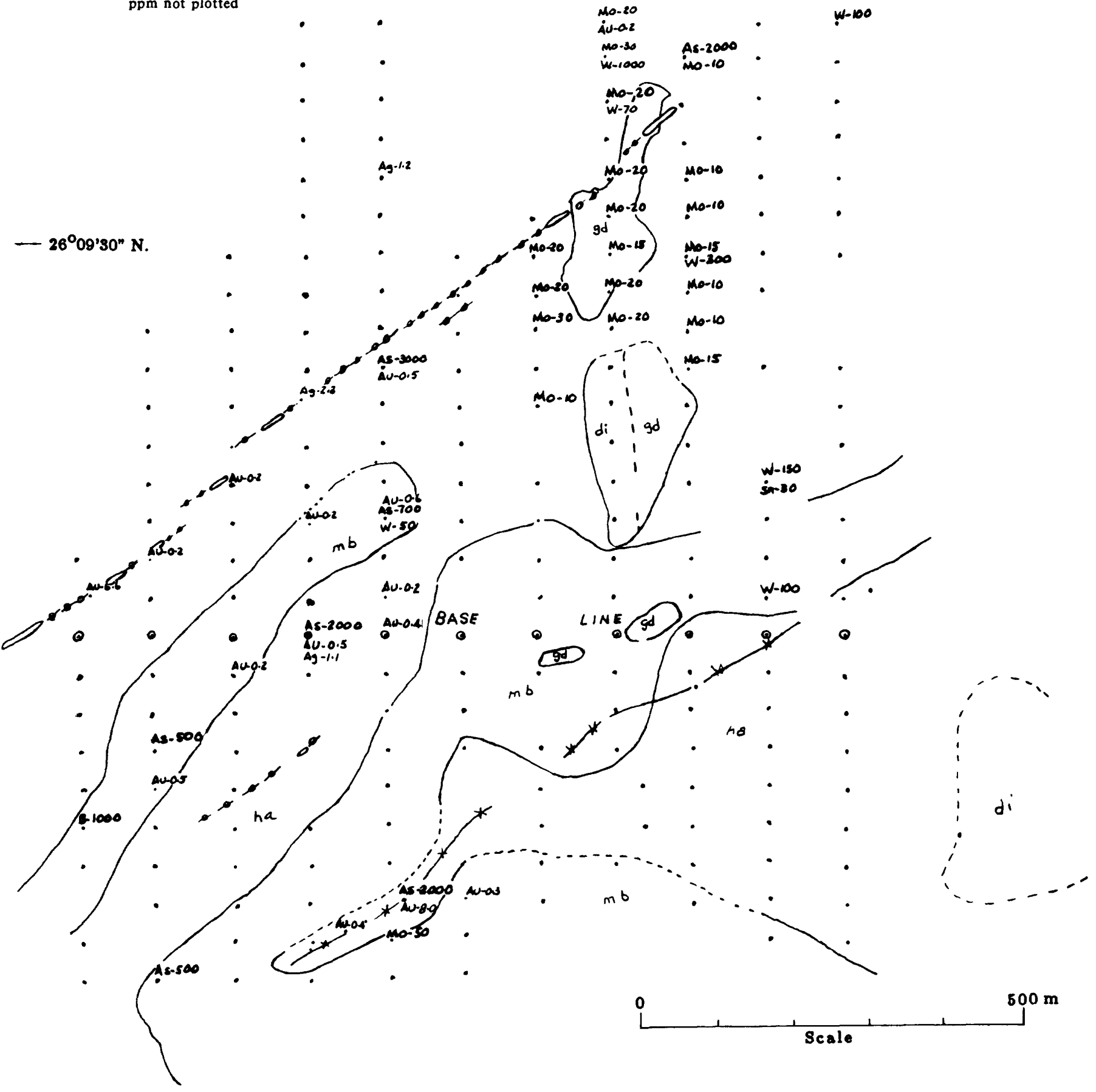

Figure 5.--Geochemical map of the Meshaheed area 
Arsenic is present in amounts as much as $3000 \mathrm{ppm}$ as an accessory element in some of the samples where gold was found, but the element is not widespread. Silver in amounts greater than $1 \mathrm{ppm}$ is present in only three samples, and one of these is from ancient dump material. Molybdenum in the $10-20 \mathrm{ppm}$ range was found in samples from the granodiorite and surrounding area at Quartz Hill No. 1 , and tungsten in the range $50-1000 \mathrm{ppm}$ is distributed erratically, mostly in contact metamorphosed metasediments in the same area. One sample of basalt from the west part of the map area contains $1000 \mathrm{ppm}$ boron, and one sample of limey metasediments southeast of Quartz Hill No. 1 contains $30 \mathrm{ppm}$ tin.

\section{ANCIENT PLACER WORKINGS}

The prominent Basalt $H i l l$ in the south part of the map area sheds talus northward over much of the map area and several branches of the wadi drain the talus slopes (fig. 2). During our previous study (Smith and Samater, 1984) ancient workings were found in talus material high up on the slope, as well as downslope in combined talus and wadi gravels. At the time these were considered to have resulted from removal of talus material to work the underlying bedrock. During the present study, however, it was concluded that the workings were the result of a search for gold in placers, since all of the worked areas are adjacent to, or on drainages. However, most of the talus and alluvium, obviously derived from Basalt Hill, is composed of coarse, angular fragments that are relatively unworked by sedimentary processes. From this, it would follow that such material is probably poor in placer gold, unless the source rock is very rich in the element.

Three trenches were excavated to a depth of about $3 \mathrm{~m}$ in the placer workings, two south of point 0,0 , and one east of 600 east (fig. 2). None of the trenches reached bedrock. Two samples were collected from the walls and bottoms of each of the trenches. Heavy minerals were separated and the magnetic fraction removed. The remaining particles were then examined under a binocular microscope, but no gold was observed.

Ancient miners worked several placer areas in Saudi Arabia, one of which is Jabal Mokhyat in the southern Najd province. Schmidt and others (1981), who very thoroughly trenched and sampled the worked placer gravels at Mokhyat, found that they contain 40 milligrams gold per cubic meter over an area of about $1 \mathrm{~km}^{2}$ and to a depth of about $1 \mathrm{~m}$. Bagdady and others (1978) trenched and sampled previously worked placer gravels at Mahd adh Dhahab where they calculated 710,910 cubic meters to average 102 milligrams per cubic meter. Ancient placer workers, well aware that free gold in placer material is much more easily recoverd than that enclosed in quartz or rock, were probably inclined to work placer material very low in gold content. As a result, it is concluded that the placers at Meshaheed are of little economic significance. However, they do indicate a gold source from Basalt Hill, only a short distance south. 


\section{TRENCHING IN BEDROCK}

Four trenches were excavated in the large hydrothermally altered zone, two on a gold-stibnite-quartz vein, and two in hydrothermally altered rock. The four trenches, designated as $T-1$ through $T-4$ (fig. 2 ), are described as follows:

T-1--This trench was excavated on the projection of a vein partially exposed in a small ancient working. The trench, located $40 \mathrm{~m}$ southwest of the working, intersected a stibnite vein containing very little quartz. Vein walls are intensely kaolinized and stained with manganese and iron oxides. No additional veins were intersected in the 16-m-long trench. Intensely kaolinized metasedimentary rock is exposed at the south end of the trench, and is more siliceous with abundant disseminated pyrite at the north end. Three samples were chipped along the trench walls and the analyses are shown in table 2.

T-2--This trench is located $125 \mathrm{~m}$ southwest of $\mathrm{T}-1$ on the projection of the vein. The trench, $9 \mathrm{~m}$ long, cut the top of a vein assumed to be the same as that intersected in $\mathrm{T}-1$. The vein, only a few centimeters of which were

exposed in the bottom of the trench, is composed of quartz with only minor stibnite. In the remainder of the trench the rock is variably altered and consists of kaolinized rock interspersed with silicified zones with accompanying manganese oxide. Rocks are highly sheared in the north end of the trench. Five samples were chipped along the trench walls and the analyses are shown in table 2.

T-3--This trench was dug in order to improve exposure of rock alteration. Sheared and kaolinized rock is cut by hematitic bands and late calcite stringers on the west end of the trench. This zone grades into carbonatized rock with abundant disseminated pyrite on the east end. Two samples were collected and analyses are shown in table 2 .

T-4--This trench was dug in the altered zone at the point where it passes beneath alluvium to the northeast (fig. 3). Alternating kaolinized zones and manganese-oxide-stained siliceous zones exposed in this trench are similar to those exposed in other trenches. Some of the rock is highly sheared and major faults are stained by manganese or iron oxides. Four samples were chipped along the trench walls and the analyses are shown in table 2.

Four additional trenches were excavated across a series of ancient pits previously named Pluton Southeast ancient workings, located about $2 \mathrm{~km}$ west of the study area (Smith and Samater, 1984). Neither workings nor trenches are shown on maps in this report. None of the trenches intersected veins, but 5- to $10-\mathrm{cm}$-thick fault gouges were cut in two of the excavations. Rocks in the trenches are mostly flinty, black hornfelsic metasediments. This group of ancient workings is $600 \mathrm{~m}$ long, as much as $40 \mathrm{~m}$ wide, and it appears that much of the effort by ancient miners may have been in prospecting, because our trenches were dug below the bottom of several ancient pits, only to find barren rock. Three samples were collected in the trenches.

Vein samples from trenches $\mathrm{T}-1$ and $\mathrm{T}-2$ indicate that gold probably persists for $175 \mathrm{~m}$ along the vein. In trench $\mathrm{T}-1$, a sample of the vein, composed mainly of stibnite, contained $14.4 \mathrm{ppm}$ gold, and a $1.5-\mathrm{m}-10 \mathrm{ng}$ sample of the vein walls had $20.6 \mathrm{ppm}$ gold. In trench $\mathrm{T}-2$, the vein was exposed only in the bottom of the trench, but a $2 \mathrm{~m}$ sample chipped above and across its projection ran $7.1 \mathrm{ppm}$ 
gold. It is possible that the gold found in vein selvages is the result of secondary enrichment, although four samples chipped across hydrothermally altered rock in trench $T-2$ are in the range $0.1-0.3 \mathrm{ppm}$ gold with arsenic in the range 120-1300 ppm. However, all other trench sampling of altered rock found no gold, minor silver, and only low values in accessory elements. 


\section{GROUND FOLLOW-UP STUDIES OF GEOPHYSICAL ANOMALIES}

Previous geophysical studies employing audio-magneto-telluric and telluric-electric methods found an anomalous zone coinciding with the northeast-striking hydrothermally altered zone at Meshaheed (Flanigan and Zablocki, 1984; Smith and Samater, 1984). The telluric-electric method also found a zone of extremely low resistivity about one kilometer east of Meshaheed. Rocks in the anomalous area (not shown in fig. 2) were examined and it was found that a small diorite intrusive into metasediments is located a few meters north of the geophysical traverse line and that its southward projection coincides with the anomalous zone. Small, siliceous gossans were found at the contact of the diorite with metasediments, but two samples of the material (200987 and 200988) found no significant metals (table 2). The small pluton is elongated north-south and may have intruded along a fault zone of the same trend. Metasediments along the trend are not obviously hydrothermally altered and the anomaly probably does not represent a zone of hydrothermal alteration with accompanying metallization. 


\section{CONCLUSIONS AND RECOMMENDATIONS}

The objective of the rock-chip sampling program was to locate extensive areas of gold-bearing rock, but these were not found. Gold deposition at Meshaheed was apparently confined to quartz veins and selvages. Hydrothermally altered areas in both metasediments and basalt are relatively barren of gold. These zones are, in fact, nearly free of trace elements such as silver, copper, lead, zinc, arsenic, molybdenum, or antimony at points removed from the veins.

One sample of altered metasediments in the selvage of the large diorite dike that crosses the map area contained $8.0 \mathrm{ppm}$ gold, but subsequent check sampling along the dike found very little of that element. Gold deposition may have occurred along the contacts of dikes and small plutons with metasediments in the Meshaheed area, but as indicated by these studies, much of the deposition was local and chances of finding large volumes of gold-bearing rock are remote.

Anomalous lead, copper, boron, and tin found by previous wadi sediment sampling in the Meshaheed area were not duplicated by the rock-chip sampling. On the contrary, in general, the rock-chip sampling found only low quantities of these metals. The source of the anomalous metals may have derived from a locality other than the present study area, or from vein erosion, but further exploration would probably not be beneficial and is not recommended.

Previous ground geophysical studies employing audio-magneto-telluric and telluric-electric methods have demonstrated their effectiveness in locating zones of low resistivity that coincide with hydrothermally altered rocks at Meshaheed. Although the altered zones tested by these geophysical methods at Meshaheed are essentially devoid of widespread metallization, in other areas similar studies may be successful in locating altered zones coinciding with such metallization.

Molybdenite-bearing quartz stockworks at Quartz Hill No. 1 are low grade with molybdenum generally assaying less than $100 \mathrm{ppm}$. The molybdenum content should be ten to twenty times greater for commercial application. Therefore, further exploration for this metal at Quartz Hill No. 1 is not recommended.

A few samples from the vicinity of Quartz Hill No. 1 contain tungsten in the range $70-1000 \mathrm{ppm}$ (fig. 5). Most of the samples containing tungsten are from contact metamorphosed limey metasediments. Tungsten minerals were not recognized during field studies, but it is possible that scheelite may be present; examination of the area by ultra-violet light is advisable.

In conclusion, these studies found that gold in the Meshaheed area is essentially confined to gold-stibnite-quartz veins. The long northeast-trending vein that intersects Quartz Hill No. 1 probably offers the greatest gold potelltial, but much more work would be required to learn details of its thickness, continuity, and average gold grade. Trenching in the Pluton Southeast group of ancient workings (location not shown in this report) has demonstrated clearly that much of the work performed by the ancients was probably that of prospecting since many of the ancient pits appear to be in barren rock. The Meshaheed area probably does not contain sufficient tonnages of commercial grade gold to stand alone as a mining operation, but may prove viable if combined with mining at nearby deposits. 
Table 1.--Analytical data for rock chip sampling in the Meshaheed area

[Values for $\mathrm{Fe}, \mathrm{Mg}, \mathrm{Ca}, \mathrm{Ti}$, and $\mathrm{Mn}$ in per cent; all remaining elements in parts per million. ' $S$ indicates semi-quantitative spectrographic assay; $A A$ indicates atomic absorption assay. For atomic absorption assays, $P$ following element indicates partial digestion of sample. Az preceding element indicates use of the $A$ to $Z$ method of sample digestion. $G$ indicates element found in greater amounts than value given; $L$ indicates element detected, but in amounts less than value given; $N$ indicates element not detected at value given.]

PRINTOUT FOR SPEC/AA

\begin{tabular}{|c|c|c|c|c|c|c|c|c|c|c|}
\hline SAMFLLE & S-FE & S-MG & $S-C A$ & S-II & S-KN & S-AG & S-AS & S-All & $S-\beta$ & S-BA \\
\hline $\begin{array}{l}200748 \\
200749\end{array}$ & $\begin{array}{r}10.0000 \\
7.0000\end{array}$ & $\begin{array}{l}5.0000 \\
1.0000\end{array}$ & $\begin{array}{l}7.0000 \\
2.0000\end{array}$ & $\begin{array}{l}0.2000 \\
0.2000\end{array}$ & $\begin{array}{l}1000.0000 \\
1000.0000\end{array}$ & $\begin{array}{l}0.5000 \mathrm{~N} \\
0.5000 \mathrm{~N}\end{array}$ & $\begin{array}{l}200.0000 \mathrm{~N} \\
200.0000 \mathrm{~N}\end{array}$ & $\begin{array}{l}10.0000 \mathrm{~N} \\
10.0000 \mathrm{~N}\end{array}$ & $\begin{array}{l}10.0000 \\
20.0000\end{array}$ & $\begin{array}{l}700.0000 \\
300.0000\end{array}$ \\
\hline 200750 & 5.0000 & 7.0005 & 5.0200 & 0.2000 & 1000.0000 & $0.5000 \mathrm{~N}$ & 700.0000 & $10.0000 \mathrm{~N}$ & 20.0000 & 150.0000 \\
\hline$: 00751$ & 5.0000 & 7.0000 & 20.0000 & 0.2000 & 2000.0000 & $0.5000 \mathrm{~N}$ & $200.0000 \mathrm{~N}$ & $10.0000 \mathrm{H}$ & 20.0000 & 700.0000 \\
\hline 200752 & .5 .0000 & 5.0000 & 5.0000 & 0.2000 & 1500,0000 & 0.500011 & $200.0000 \mathrm{~N}$ & $10.0000 \mathrm{~N}$ & 50.0000 & 500.0000 \\
\hline 200753 & 5.0000 & 10.0000 & 1.0000 & 0.3000 & 700.0000 & $0.5000 \mathrm{~N}$ & $200.0000 \mathrm{~N}$ & $10.0000 \mathrm{~N}$ & 20.0000 & 700,0000 \\
\hline 200754 & 5.0000 & 7.0001 & 20.0000 & 0.2000 & 2000.0000 & $0.5000 \mathrm{~N}$ & $200.0000 \mathrm{~N}$ & $10.0000 \mathrm{~N}$ & 30.0000 & 500.0000 \\
\hline 200755 & 2.0000 & 0.1500 & 2,0000 & 0.0200 & 700.0000 & $0,5000 \mathrm{~N}$ & 3000.0000 & $10.0000 \mathrm{H}$ & 20.0000 & 150,0000 \\
\hline 200756 & 5.0000 & 5.0000 & 5.0000 & 0.2000 & 1000.0000 & $0.5000 \mathrm{~N}$ & $200.0000 \mathrm{~N}$ & $10.0000 \mathrm{~N}$ & 30.0000 & 500.0000 \\
\hline $\begin{array}{l}200757 \\
200758\end{array}$ & $\begin{array}{l}5.0000 \\
5.0000\end{array}$ & $\begin{array}{l}7.0000 \\
5.0000\end{array}$ & $\begin{array}{r}: 5.0000 \\
\therefore .0000\end{array}$ & $\begin{array}{l}0.2000 \\
0.2000\end{array}$ & $\begin{array}{l}2000.00000 \\
1000.0000\end{array}$ & $\begin{array}{l}0.5000 \mathrm{~N} \\
0.5000 \mathrm{~N}\end{array}$ & $\begin{array}{l}200.0000 \mathrm{~N} \\
200.0000 \mathrm{~N}\end{array}$ & $\begin{array}{l}10.0000 \mathrm{~N} \\
10.0000 \mathrm{H}\end{array}$ & $\begin{array}{l}30.0000 \\
50.0000\end{array}$ & $\begin{array}{l}700.0000 \\
500.0000\end{array}$ \\
\hline 200759 & 5.0000 & 5.0000 & 1,0000 & 0.2000 & 200.0000 & $0.5000 \mathrm{~N}$ & $200.0000 \mathrm{~N}$ & $10.0000 \mathrm{~N}$ & 70.0000 & 700.0000 \\
\hline $\begin{array}{l}206750 \\
206781\end{array}$ & $\begin{array}{l}5.0000 \\
3.0000\end{array}$ & $\begin{array}{l}5.0000 \\
5.0000\end{array}$ & $\begin{array}{r}5.0000 \\
24.0000\end{array}$ & $\begin{array}{l}0.2000 \\
0.1500\end{array}$ & $\begin{array}{l}1500.0000 \\
1000.0000\end{array}$ & $\begin{array}{l}0.5000 \mathrm{H} \\
0.5000 \mathrm{~N}\end{array}$ & $\begin{array}{l}200.0000 \mathrm{~N} \\
200.0000 \mathrm{H}\end{array}$ & $\begin{array}{l}10,0000 \mathrm{~N} \\
10,0000 \mathrm{~N}\end{array}$ & $\begin{array}{l}30.0000 \\
10.00001\end{array}$ & $\begin{array}{l}700.0000 \\
500.0000\end{array}$ \\
\hline 200762 & 7.0000 & $\therefore 0003$ & 1.5000 & 0.2000 & 700.0000 & $0.5000 \mathrm{~N}$ & $200.0000 \mathrm{~N}$ & $10.00 \mathrm{CON}$ & 20,0000 & 500.0000 \\
\hline 200743 & 5,0000 & 5.0000 & 1.0000 & 0.2000 & 700.0000 & $0.5000 \mathrm{~N}$ & $200.0000 \mathrm{~N}$ & 10.000011 & 30.0000 & 1000.0000 \\
\hline 200764 & 5.0000 & 7.0000 & 0.7000 & 0.2000 & 700.0000 & $0.5000 \mathrm{~N}$ & $200.0000 \mathrm{H}$ & 10.000014 & 30.0000 & 700.0000 \\
\hline 200765 & 5.0000 & 5.0000 & 0.5000 & 0.2000 & 500.0000 & $0.5000 \mathrm{~N}$ & $200.0000 \mathrm{~N}$ & $10.0000 \mathrm{~N}$ & 50.0000 & 700.0000 \\
\hline 200766 & 5.0000 & 5.0000 & 0.5000 & 0.2000 & 500.0000 & $0.5000 \mathrm{~N}$ & $200.0000 \mathrm{~N}$ & $10.0000 \mathrm{H}$ & 70.0000 & 700.0000 \\
\hline 200767 & & & & 0.5000 & 2000.0000 & $0.5000 \mathrm{~N}$ & $200.0000 \mathrm{~N}$ & $10.0000 \mathrm{H}$ & 30.0000 & 200.0000 \\
\hline 200268 & 10.0000 & 1.0000 & 2.0000 & 0.1500 & 500.0000 & 0.5000 & $200.0000 \mathrm{~N}$ & $10.0000 \mathrm{~N}$ & 20.0000 & 70.0000 \\
\hline 200769 & 5.0000 & 10.0000 & 5.0000 & 0.3000 & 500,0000 & $0.5000 \mathrm{~N}$ & $200.0000 \mathrm{~N}$ & $10,0000 \mathrm{~N}$ & 10.0000 & 100.0000 \\
\hline 200770 & 5.0000 & 5.0000 & 5.0000 & 0.2000 & 700.0000 & $0.5000 \mathrm{~N}$ & $200.0000 \mathrm{~N}$ & $10.0000 \mathrm{~N}$ & 10.00001 & $20.0000 \mathrm{~N}$ \\
\hline 200771 & 5.0000 & 5.0000 & 0.7000 & 0.2000 & 500.0000 & $0.5000 \mathrm{~N}$ & $200.0000 \mathrm{~N}$ & $10.0000 \mathrm{H}$ & 30.0000 & 500.0000 \\
\hline 200772 & 10.0000 & 0.1000 & 2.0000 & 0.0500 & 150.0000 & 0.50001 & 2000.0000 & $10.0000 \mathrm{~N}$ & 30.0000 & 70.0000 \\
\hline 200773 & 15.0000 & 0.2000 & 0.50 & 0.1000 & 150.0000 & $0.5000 \mathrm{~L}$ & $200.0000 \mathrm{~N}$ & $10.0000 \mathrm{H}$ & 30.0000 & 100.0000 \\
\hline 200774 & 7.0000 & 0.5020 & 3.0000 & 0.2000 & 700.0000 & $0.5000 \mathrm{~L}$ & $200.0000 \mathrm{~N}$ & $10.0000 \mathrm{~N}$ & 10.0000 & 150.0000 \\
\hline 200775 & 7.0000 & 0.5000 & 0.5000 & 0.2000 & 500.0000 & 1.0000 & 2000.0000 & $10.0000 \mathrm{H}$ & 30.0000 & 500.0000 \\
\hline 200776 & 7.0000 & 0.5000 & 1.0000 & 0.1000 & 300.0000 & 1.0000 & 200.000011 & $10.0000 \mathrm{~N}$ & 20.0000 & 200.0000 \\
\hline 200777 & 5.0000 & 7.0000 & 5.0000 & 0.3000 & 1000.0000 & $0.5000 \mathrm{~L}$ & $200.0000 \mathrm{~N}$ & $10.0000 \mathrm{~N}$ & $10.0000 \mathrm{~L}$ & 200.0000 \\
\hline 200778 & 5.0000 & 5.0000 & 1.0000 & 0.2000 & 500.0000 & $0.5000 \mathrm{~L}$ & $200.0000 \mathrm{~N}$ & $10.0000 \mathrm{H}$ & 15.0000 & 200.0000 \\
\hline 200779 & 0000 & 7,0000 & 2.0000 & 0.3000 & 700.0000 & $0.5000 \mathrm{~L}$ & $200.0000 \mathrm{~N}$ & $10.0000 \mathrm{~N}$ & 10.0000 & 700.0000 \\
\hline 200780 & 5.0000 & 5.0000 & 1.0000 & 0.2000 & 500.0000 & $0.5000 \mathrm{~L}$ & $200.0000 \mathrm{~N}$ & $10.0000 \mathrm{~N}$ & 30.0000 & 500.0000 \\
\hline 200781 & 5.0000 & 5.0000 & 0.7000 & 0.2000 & 500.0000 & $0.5000 \mathrm{~N}$ & $200.0000 \mathrm{~N}$ & $10.0000 \%$ & 20.0000 & 300.0000 \\
\hline 200782 & 3000 & & 0.7 & .00201 & 200.0 & 2.0000 & $200.0000 \mathrm{~N}$ & $10.0000 \mathrm{~N}$ & 10.0000 & 50.00000 \\
\hline 200783 & 1.5000 & 2.0000 & 5.0000 & 0.1500 & 700.0000 & $0,5000 \mathrm{~N}$ & 200.000004 & 10.000014 & 10.00001 & 700.0000 \\
\hline 200784 & 5.0000 & 2.0000 & 5,0000 & 0.2000 & 500.0000 & $0.5000 \mathrm{~N}$ & $200.0000 \mathrm{~N}$ & $10.0000 \mathrm{H}$ & 20.0000 & 500.0000 \\
\hline 200795 & 5.0000 & 2.0000 & 0.5000 & 0.2000 & 200.0000 & $0.5000 \mathrm{~N}$ & $200.0000 \mathrm{~N}$ & $10,0000 \mathrm{~N}$ & 30.0000 & 500.0000 \\
\hline 200786 & 5.0000 & 3.0000 & 0.5000 & 0.2000 & 200.0000 & $0.5000 \mathrm{~N}$ & $200.0000 \mathrm{~N}$ & 10.000014 & 50.0000 & 500,0000 \\
\hline $\begin{array}{l}200787 \\
200788\end{array}$ & $\begin{array}{l}3.0000 \\
3.0000\end{array}$ & $\begin{array}{l}1.5000 \\
3.0000\end{array}$ & $\begin{array}{l}5.0000 \\
2.0000\end{array}$ & $\begin{array}{l}0.1500 \\
0.2000\end{array}$ & $\begin{array}{l}500.0000 \\
200.0000\end{array}$ & $\begin{array}{l}0.5000 \mathrm{~N} \\
0.5000 \mathrm{~N}\end{array}$ & $\begin{array}{l}200.0000 \mathrm{H} \\
200.0000 \mathrm{~N}\end{array}$ & $\begin{array}{l}10.0000 \mathrm{~N} \\
10.0000 \mathrm{~N}\end{array}$ & $\begin{array}{l}10.0000 \\
30.0000\end{array}$ & $\begin{array}{l}150.0000 \\
700.0000\end{array}$ \\
\hline 200789 & 5.0000 & 5.0000 & 0.5000 & 0.2000 & 300.0000 & $0.5000 \mathrm{~N}$ & $200.0000 \mathrm{~N}$ & 10.00001 & 20.0000 & 700.0000 \\
\hline 200790 & 5.0000 & & & 0.2000 & 500,0000 & $0.5000 \mathrm{~N}$ & $200.0000 \mathrm{~N}$ & $10.0000 \mathrm{H}$ & 20.0040 & 700.0000 \\
\hline 200791 & 3.0000 & 7.0000 & 20.0000 & 0.1000 & 1500.0000 & $0,5000 \mathrm{~N}$ & $200.0000 \mathrm{~N}$ & $10.0000 \mathrm{~N}$ & 20.0000 & 500.0000 \\
\hline 200992 & 3.0000 & 2.0000 & 20.0000 & 0.2000 & 2000.0000 & $0.5000 \mathrm{~N}$ & $200.0000 \mathrm{~N}$ & $10.0000 \mathrm{~N}$ & 30.0000 & 700.0000 \\
\hline 200793 & 5.0000 & 3.0000 & 0.3000 & 0.2000 & 500.0000 & $0.5000 \mathrm{~N}$ & $200,0000 \mathrm{~N}$ & $10,0000 \mathrm{~N}$ & 30,0000 & 150.0000 \\
\hline 200794 & 5.0000 & 2.0000 & 0.7000 & 0.2000 & 500.0000 & $0.5000 \mathrm{~N}$ & $200.0000 \mathrm{~N}$ & 10.000011 & 30.0000 & 500.0000 \\
\hline 200795 & 7.0000 & 10,0000 & 5.0000 & 0.5000 & 1500.0000 & $0.5000 \mathrm{~N}$ & $200,0000 \mathrm{~N}$ & $10.0000 \mathrm{~N}$ & 20.0000 & 100.0000 \\
\hline 200796 & 7.0000 & 10.0006 & 5.0000 & 0.5000 & 1500,0000 & $0.500 \mathrm{CN}$ & $200.0000 \mathrm{~N}$ & $10.0000 \mathrm{~N}$ & 20.0000 & 200.0000 \\
\hline 200797 & 5.0000 & 7.0000 & 7,0000 & 0.5000 & 1500.0000 & $0.5000 \mathrm{H}$ & $200,0000 \mathrm{~N}$ & $10.0000 \mathrm{~N}$ & 20.0000 & 150.0000 \\
\hline
\end{tabular}


Table 1.--Analytical data for rock chip sampling--Continued

\author{
PRINTOUT FOR SFEC/AA
}

\begin{tabular}{|c|c|c|c|c|c|c|c|c|c|c|}
\hline YFLE & BE & $S-B I$ & $-c a$ & $S-C D$ & $-C R$ & $s-c U$ & $S-L A$ & $-H_{0}$ & $S-M B$ & S-NI \\
\hline $\begin{array}{l}200748 \\
260749\end{array}$ & $\begin{array}{l}1.0000 \\
1.0000\end{array}$ & $\begin{array}{l}10.0000 \mathrm{~N} \\
10.0000 \mathrm{~N}\end{array}$ & $\begin{array}{l}20.0000 \mathrm{~N} \\
20.0000 \mathrm{~N}\end{array}$ & $\begin{array}{l}30.0000 \\
10.0000\end{array}$ & $\begin{array}{l}150.0000 \\
150.0000\end{array}$ & $\begin{array}{l}70.0000 \\
10.0000\end{array}$ & & $\begin{array}{l}5.0000 \mathrm{~N} \\
5.0000 \mathrm{~N}\end{array}$ & $\begin{array}{l}.00006 \\
.00606\end{array}$ & $\begin{array}{l}70.0000 \\
50.0000\end{array}$ \\
\hline 200750 & 1.0000 & $10.0000 \mathrm{~N}$ & 20.000011 & 50.0000 & 150,0000 & 50.0000 & 30.0000 & $5.0000 \mathrm{~N}$ & 20.00001 & 70.0000 \\
\hline $\begin{array}{l}200751 \\
200752\end{array}$ & $\begin{array}{l}1.0000 \\
1.0000\end{array}$ & $\begin{array}{l}10.000011 \\
10.000011\end{array}$ & $\begin{array}{l}20.0000 \mathrm{~N} \\
20.0000 \mathrm{~N}\end{array}$ & $\begin{array}{l}30.0000 \\
20.0000\end{array}$ & $\begin{array}{r}1000.0000 \\
200.0000\end{array}$ & $\begin{array}{l}15.0000 \\
15.0000\end{array}$ & & $\begin{array}{l}5.000011 \\
5.0000 \mathrm{~N}\end{array}$ & $\begin{array}{l}20.0000 \mathrm{~L} \\
20.000 \mathrm{CL}\end{array}$ & $\begin{array}{l}70.0000 \\
50.0000\end{array}$ \\
\hline 200753 & 1.0600 & 10.000011 & 20.000014 & 30.0000 & 200.0000 & 50.0000 & 30.0000 & $5.0000 \mathrm{~N}$ & $20.0000 \mathrm{~L}$ & 0.0000 \\
\hline 0754 & & & & & & & & & & \\
\hline 200755 & 1.0000 & & $.0000 \mathrm{~N}$ & $5.0000 \mathrm{~N}$ & 000 & 7.0000 & $.0000 \mathrm{~N}$ & OON & $000 \mathrm{~L}$ & .0000 \\
\hline 200756 & 1.0000 & 10.000011 & $00 \mathrm{~N}$ & 50.0000 & 300 & 15.0000 & & & 0000 & 0000 \\
\hline 200757 & & & & & & & & & & 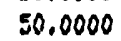 \\
\hline 200758 & 1.0 & & & & & & & & 1002 & .0000 \\
\hline 200753 & & & OON & & 000 & & & OOOH & 3006 & .0000 \\
\hline 200760 & & & & & 100 & & & & & \\
\hline 200761 & & & & & & & & & & \\
\hline 200762 & 1.0000 & 6 & 0011 & 30. & 200 & & & & & .0000 \\
\hline 200703 & 1 & & OON & & & & & & & .0000 \\
\hline & & & & & & & & & & \\
\hline 200765 & & & & & & & & & & 0000 \\
\hline $200760^{\circ}$ & a & & ON & 20. & & & & & & \\
\hline 200767 & & & & & & & & & & \\
\hline 200769 & $0 n$ & 10. & 0011 & 20.0000 & 100 & & & & & 0000 \\
\hline 200769 & $1.0000 \mathrm{~N}$ & & Oo:1 & 30 & 500 & & OOH & & & .0000 \\
\hline 200770 & & & & & 200 & & & & & 1000 \\
\hline 200771 & & & & & & & & & & 1000 \\
\hline 200772 & & & & & & & & & & 000 \\
\hline 200773 & & & & OL & & & & & & 000 \\
\hline 200774 & ON & & & 50.0000 & 500.0000 & 00 & ON & & & .0000 \\
\hline 200775 & & & & & & & & & & 1000 \\
\hline 200778 & & & & & & & & & & 000 \\
\hline 200777 & $\mathrm{~N}$ & 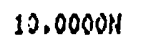 & & 0 & 500 & & & & OON & .0000 \\
\hline 200 & & & & & & & & & & \\
\hline & & & & & & & & & & \\
\hline 200780 & $0060 \mathrm{~N}$ & ON & SN & 20. & 300.0000 & 00 & & & & 50.0000 \\
\hline m & & & & & & & & & & \\
\hline & & & & & & & & & & \\
\hline 200783 & ION & & & & & & & & & 000 \\
\hline & & & & & & & & & & 0000 \\
\hline & & & & & & & & & & 000 \\
\hline 300786 & $M$ & $O H$ & & 00 & 100 & & & & & 70.0000 \\
\hline & $1.0000 \mathrm{~N}$ & & & & 200. & & & & & 0000 \\
\hline 200788 & $1.0000 \mathrm{~L}$ & $10.0060 \mathrm{H}$ & OON & 10.0000 & 150.0000 & & & & OON & 70.0000 \\
\hline 200789 & $1.0000 \mathrm{~L}$ & & & 20.0000 & & & & & & 70.0000 \\
\hline & & & & & & & & & & 0000 \\
\hline & & & & & 150. & & & & OOH & 30.0000 \\
\hline 200792 & 00006 & $10.0000 \mathrm{~N}$ & OOOH & 10.0 & 150 & & & & 00111 & 50.0000 \\
\hline & & & & & & & & & & \\
\hline & & & & & & & & & OON & 50.0000 \\
\hline & & & & & & & & & ON & 0000 \\
\hline 20 & $O L$ & ON & & 50,0000 & 200. & & & & & 50. \\
\hline & 1,60001 & $10.0000 \mathrm{~N}$ & $20.0000 \mathrm{~N}$ & 50.0000 & 200.0000 & 50.0000 & 20.0000 & $5.0000 \mathrm{~N}$ & $20.0000 \mathrm{~N}$ & 70.0000 \\
\hline
\end{tabular}


Table 1.--Analytical data for rock chip sampling--Continued

PRINTOUT FOR SPECAAA

\begin{tabular}{|c|c|c|c|c|c|c|c|c|c|c|}
\hline SAFFLE & $P B$ & $5-5 B$ & $-S C$ & S-SN & $S-5 R$ & -4 & $5-4$ & S-Y & $s-2 M$ & $5-26$ \\
\hline $\begin{array}{l}200748 \\
200749\end{array}$ & $\begin{array}{l}10.0000 L \\
10.0000\end{array}$ & $\begin{array}{l}100.0000 \mathrm{~N} \\
100.0000 \mathrm{H}\end{array}$ & $\begin{array}{l}30.0000 \\
30.0000\end{array}$ & $\begin{array}{l}10.0000 \mathrm{~N} \\
10.0000 \mathrm{~K}\end{array}$ & $\begin{array}{l}300.0000 \\
500.0000\end{array}$ & $\begin{array}{l}200.0000 \\
100.0000\end{array}$ & $\begin{array}{l}0.0000 \mathrm{~N} \\
0.0000 \mathrm{~N}\end{array}$ & $\begin{array}{l}20.0000 \\
15.0000\end{array}$ & $\begin{array}{l}200.0000 \mathrm{H} \\
200.0000 \mathrm{~N}\end{array}$ & $\begin{array}{r}70.0000 \\
100.0000\end{array}$ \\
\hline 200750 & $10.0000 \mathrm{~L}$ & 100,000011 & 50.0000 & $10,0000 \mathrm{H}$ & 150.0000 & 200.0000 & 30.0000 & 20.0000 & $200.0000 \mathrm{~N}$ & 70.0000 \\
\hline 260751 & & & & $10.0000 \mathrm{~N}$ & & & $0000 \mathrm{~L}$ & & $200.0000 \mathrm{~N}$ & 150.0000 \\
\hline & & & & & & 10 & & & OOOCN & 200.0000 \\
\hline 200753 & & 130 & 00 & $10.0000 \mathrm{~N}$ & 300.0000 & 150.0000 & & & $200.000 \mathrm{BH}$ & \\
\hline 200754 & & & 20.0000 & $10.0000 \mathrm{~N}$ & 700.0000 & 150.0000 & $0000 \mathrm{~N}$ & 30.0000 & $200.0000 \mathrm{~N}$ & 200.0000 \\
\hline 0755 & & & & & & & SOOH & $0000 \mathrm{~L}$ & $0000 \mathrm{~N}$ & 20.0000 \\
\hline 0756 & & 200.0 & 20.0 & & 500. & 150. & & & $.0000 \mathrm{H}$ & 150.0000 \\
\hline $75 ?$ & & & & & & & & & & \\
\hline 758 & & & & & & & & & $000 \mathrm{~N}$ & 150,0000 \\
\hline 0759 & & 100. & & & OOON & & & 000 & OOON & 200.0000 \\
\hline 700 & & & 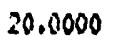 & OON & & & & & OOOON & 3000 \\
\hline & & & & & & & & & & \\
\hline & & & & & & & & & OON & 000 \\
\hline & & & & & & & & & & \\
\hline$\$ 4$ & & & 6 & & & & & & OON & \\
\hline 5 & & & & & & & & & $O C N$ & \\
\hline 766 & & & & & & & & & & \\
\hline 1767 & & & & & & & & & 2011 & \\
\hline & & & & & OON & & & & & \\
\hline 200769 & & & & & & & & & & 000 \\
\hline ד הירה & & & & & & & & & & \\
\hline 0771 & & & & & & & & & & \\
\hline 2 & & & & & & & & & & \\
\hline & & & & & & & & & & \\
\hline 00774 & & 100 & 30. & & & 00 & & & & 000 \\
\hline & & & & & & & & & & \\
\hline 776 & & & & & & & & CON & SOH & \\
\hline 77 & & & & & & & & & & \\
\hline & & & & & & & & & & \\
\hline & & & & & & & & & & \\
\hline & & & & & & & & & & \\
\hline & & & & & & & & & & \\
\hline & & & & & & & & & & 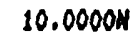 \\
\hline & & & & & & & $\mathrm{ON}$ & & OON & 000 \\
\hline & & & & & & & & & & \\
\hline & & & & & 001 & & & & OOH & 000 \\
\hline & & & & & & & & & $000 \mathrm{~N}$ & \\
\hline & & & & & & & & & & \\
\hline 0789 & & & & & & & & & & \\
\hline & & & & & & & & & & \\
\hline & & & & & & & & & & \\
\hline 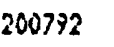 & & & & & & 00 & & & OON & \\
\hline & & & & & & & & & & \\
\hline & & & & & & & & & & \\
\hline & & & & & & & & & & \\
\hline$m$ & & 100. & & & 200.0000 & & & .30 .0000 & 0000N & 100.0000 \\
\hline & 30.00006 & $100.0000 \mathrm{~N}$ & 50.0000 & $10.0000 \mathrm{~N}$ & 150.0000 & 200.0000 & $50.0000 \mathrm{~N}$ & 30.0000 & $200.0000 \mathrm{H}$ & 100.0 \\
\hline
\end{tabular}


Table 1.--Analytical data for rock chip sampling--Continued

FRIMTOUT FOR SPEC/AA

\begin{tabular}{|c|c|c|}
\hline SAHFLE & $A A-A U-F$ & $A I-A G$ \\
\hline 200748 & 0.1600 & 0.1500 \\
\hline 200749 & $0.0500 \mathrm{~N}$ & 0.1700 \\
\hline 200750 & 0.5600 & 0.2700 \\
\hline 204151 & $0.0500 \mathrm{~N}$ & $0.1000 \mathrm{~L}$ \\
\hline 200752 & $0.0500 \mathrm{~N}$ & 0.10002 \\
\hline 200753 & 0.050 @i & $0.1000 \mathrm{~N}$ \\
\hline 200754 & $0.0500 \mathrm{~N}$ & 0.1000 \\
\hline 200755 & 0.5300 & 0.4700 \\
\hline 200756 & 0.05002 & 0.1100 \\
\hline 200757 & 0.3800 & 0.1500 \\
\hline 200758 & $0.0500 \mathrm{~N}$ & $0.1060 \mathrm{~L}$ \\
\hline 200759 & 0.05004 & 0.10002 \\
\hline 200766 & 0.0600 & $0.1000 \mathrm{~L}$ \\
\hline 200731 & $0.0500 \mathrm{H}$ & 1.1600 \\
\hline 200762 & 0.05004 & 0.1000 \\
\hline 200763 & $0.0500 \mathrm{~L}$ & 0.10002 \\
\hline 200764 & $0.0500 \mathrm{~N}$ & $0.1000 \mathrm{~L}$ \\
\hline 200765 & 0.1000 & $0.1000 L$ \\
\hline 200766 & $0.0500 \mathrm{~N}$ & $0.1000 \mathrm{~L}$ \\
\hline 200767 & $0.0500 \mathrm{~N}$ & $0.1000 \mathrm{~L}$ \\
\hline 200768 & $0.0500 \mathrm{~N}$ & 0.8700 \\
\hline 200769 & 0.0600 & 0.10002 \\
\hline 200770 & $0.0500 \mathrm{~N}$ & $0.1000 \mathrm{~N}$ \\
\hline 200771 & $0.0500 \mathrm{~N}$ & $0.1000 L$ \\
\hline 200772 & 8.0000 & 0.5000 \\
\hline 200773 & $0.0500 \mathrm{~N}$ & 0.3400 \\
\hline 200774 & $0.0500 \mathrm{~N}$ & 0.1600 \\
\hline 200775 & 0.4800 & 1.0600 \\
\hline 200776 & $0.0500 \mathrm{~N}$ & 0.8400 \\
\hline$: 00777$ & $0.0500 \mathrm{~N}$ & $0.1000 \mathrm{~L}$ \\
\hline $\begin{array}{l}200778 \\
200779\end{array}$ & $\begin{array}{l}0.3800 \\
0.0500 \mathrm{H}\end{array}$ & $\begin{array}{l}0.1800 \\
0.1000 L\end{array}$ \\
\hline 200780 & $0.0500 \mathrm{~N}$ & $0.1000 L$ \\
\hline 200781 & $0.0500 \mathrm{~N}$ & $0.1000 L$ \\
\hline 200782 & $0.0500 \mathrm{~L}$ & 2,3300 \\
\hline 200783 & 0.1000 & 0.1000 \\
\hline 200784 & $0.0500 \mathrm{~L}$ & 0.10002 \\
\hline 206785 & $0.0500 \mathrm{~N}$ & 0.1100 \\
\hline 200786 & 0.0800 & $0,1000 \mathrm{~L}$ \\
\hline 200787 & 0.0800 & 0.1800 \\
\hline 200788 & 0.1000 & $0.1000 L$ \\
\hline 200789 & 0.1200 & 0.1400 \\
\hline 200790 & $0.0500 \mathrm{~N}$ & $0.1000 L$ \\
\hline 200791 & $0.0500 \mathrm{~N}$ & 0.1200 \\
\hline 200792 & $0.0500 \mathrm{~N}$ & 0.10002 \\
\hline 200793 & $0.0500 \mathrm{H}$ & 0.100011 \\
\hline 200794 & 0.0800 & $0.1000 \mathrm{~L}$ \\
\hline 200795 & $0.0500 \mathrm{~N}$ & 0,3200 \\
\hline 260796 & $0.0560 \mathrm{~N}$ & 0.1400 \\
\hline 200797 & 0.05001 & 0.5200 \\
\hline
\end{tabular}


Table 1.--Analytical data for rock chip sampling--Continued

FRINTOUT FOR SPEC/AA

\begin{tabular}{|c|c|c|c|c|c|c|c|c|c|c|}
\hline SAMPLE & S-FE & $5-146$ & $S-C A$ & $-1 I$ & S- & S-AG & S-AS & S-AUU & $5-8$ & S-RA \\
\hline $\begin{array}{l}200798 \\
200799\end{array}$ & $\begin{array}{l}5.0000 \\
5.0000\end{array}$ & $\begin{array}{r}10.0000 \\
2.0000\end{array}$ & $\begin{array}{l}5.0000 \\
0.7000\end{array}$ & $\begin{array}{l}0.2000 \\
0.2000\end{array}$ & $\begin{array}{r}1000,0000 \\
500.0000\end{array}$ & $\begin{array}{l}0.5000 \mathrm{~N} \\
0.5000 \mathrm{~N}\end{array}$ & $\begin{array}{l}200.0000 \mathrm{~N} \\
200.0000 \mathrm{~N}\end{array}$ & $\begin{array}{l}10,0000 \mathrm{~N} \\
10.0000 \mathrm{H}\end{array}$ & $\begin{array}{l}20.0000 \\
30.0000\end{array}$ & $\begin{array}{l}200.0000 \\
700.0000\end{array}$ \\
\hline 200800 & 3.0000 & 0,3000 & 0.5000 & 0.2000 & 200.0000 & $0.5000 \mathrm{~N}$ & $200.0000 \mathrm{~N}$ & $10,0000 \mathrm{~N}$ & 30.0000 & 500.0000 \\
\hline $\begin{array}{l}200801 \\
200802\end{array}$ & $\begin{array}{l}5,0000 \\
5,0000\end{array}$ & $\begin{array}{l}7.0000 \\
1.5000\end{array}$ & $\begin{array}{l}5.0000 \\
1.0000\end{array}$ & $\begin{array}{l}0.2000 \\
0.2000\end{array}$ & $\begin{array}{l}700.0000 \\
500.0000\end{array}$ & $\begin{array}{l}0.5000 \mathrm{H} \\
0.5000 \mathrm{~N}\end{array}$ & $\begin{array}{l}200,0000 \mathrm{~N} \\
200,0000 \mathrm{~N}\end{array}$ & $\begin{array}{l}10.0000 \mathrm{~N} \\
10.0000 \mathrm{~N}\end{array}$ & $\begin{array}{l}20.0000 \\
50.0000\end{array}$ & $\begin{array}{l}200,0000 \\
500,0000\end{array}$ \\
\hline 200803 & 7.0000 & 0.2000 & 0.7000 & 0.1500 & 200.0000 & $0.5000 \mathrm{~N}$ & $200.0000 \mathrm{H}$ & $10.0000 \mathrm{~N}$ & 30.0000 & 500.0000 \\
\hline 200304 & 2.0000 & 2.0000 & 5,0000 & 0.1000 & 500.0000 & $0.5000 \mathrm{H}$ & $200.0000 \mathrm{~N}$ & $10.0000 \mathrm{~N}$ & 30.0000 & 500.0000 \\
\hline 200805 & 5.0000 & 7,0000 & 5.0000 & 0.2000 & 700,0000 & $0,5000 \mathrm{~N}$ & $200.0000 \mathrm{~N}$ & $10.0000 \mathrm{~N}$ & 20.0000 & 300.0000 \\
\hline 260806 & 5.6600 & 3.0000 & 5.0000 & 0.2000 & 700.0060 & .500011 & $200.0000 \mathrm{~N}$ & $10.0000 \mathrm{~N}$ & 30.0000 & 1000.0000 \\
\hline $\begin{array}{l}200807 \\
200808\end{array}$ & $\begin{array}{l}5.0000 \\
5.0000\end{array}$ & $\begin{array}{l}3.0000 \\
7.0000\end{array}$ & $\begin{array}{l}5,0000 \\
0.7000\end{array}$ & & & & $\begin{array}{l}200.0000 \mathrm{~N} \\
200.0000 \mathrm{~N}\end{array}$ & & & \\
\hline 200809 & 5.0000 & 5,0000 & 10.0000 & 0.3000 & 1000,0000 & $0.5000 \mathrm{~N}$ & $200.0000 \mathrm{~N}$ & $10,0000 \mathrm{H}$ & 30.0000 & 1500,0000 \\
\hline 200810 & 5.0000 & 10.0000 & 3.0000 & 0.5000 & 1000.0000 & $0.5000 \mathrm{~N}$ & $200.0000 \mathrm{~N}$ & $10.0000 \mathrm{H}$ & 30.0000 & 2000,0000 \\
\hline 200811 & 5.0000 & & 5.6 & & 1000 & & OON & GON & & 0000 \\
\hline 200812 & 5.0800 & 7.0000 & 5.0000 & 0.5000 & 1500.0000 & $0.5000 \mathrm{~N}$ & $200.0000 \mathrm{~N}$ & $10.0000 \mathrm{~N}$ & 50.0000 & 2000.0000 \\
\hline $\begin{array}{l}200813 \\
200814\end{array}$ & $\begin{array}{l}5.0000 \\
5.0000\end{array}$ & & $\begin{array}{r}3.0000 \\
20.0000\end{array}$ & & $\begin{array}{l}1500,0000 \\
2000,0000\end{array}$ & & & & & \\
\hline 200815 & 5.0600 & 5.0000 & 5.0000 & 0.5000 & 1000. & $0.5000 \mathrm{~N}$ & $200.0000 \mathrm{~N}$ & 00014 & 000 & 500.0000 \\
\hline 200916 & 7.0000 & 2,0000 & 2.0000 & 0.2000 & 500 . & $0,5000 \mathrm{~N}$ & $200.0000 \mathrm{~N}$ & OOON & 10.0000 & 500.0000 \\
\hline 200817 & 5.0000 & 1.5060 & 1.0 & 00 & 1000 & & DON & OOHS & & 0000 \\
\hline $2008: 8$ & 5.0000 & 7.0000 & 7.0000 & 000 & 2000 & $.5000 \mathrm{~N}$ & $200,0000 \mathrm{~N}$ & $0000 \mathrm{~N}$ & 1000 & 300.0000 \\
\hline $\begin{array}{l}200819 \\
200820\end{array}$ & $\begin{array}{r}7.0000 \\
10.0000\end{array}$ & & $\begin{array}{r}5.0000 \\
10.0000\end{array}$ & & $\begin{array}{l}2000.0000 \\
2000.0000\end{array}$ & & & & & $\begin{array}{l}500.0000 \\
100,0000\end{array}$ \\
\hline 200821 & & 0000 & & & & $0.5000 \mathrm{~N}$ & $200.0000 \mathrm{~N}$ & OON & 000 & 500.0000 \\
\hline 200822 & 5.0000 & 5,0000 & 00 & 0.5000 & 1000. & $0.5000 \mathrm{~N}$ & $200.0000 \mathrm{~N}$ & $10.0000 \mathrm{~N}$ & 30.0000 & 1500.0000 \\
\hline 200823 & 7.0000 & 5.0000 & 1.0000 & 0.5000 & 500.0000 & $0.5000 \mathrm{~N}$ & $200.0000 \mathrm{~N}$ & $10.0000 \mathrm{H}$ & 50.0000 & 700.0000 \\
\hline 200824 & 5.0000 & 5.0000 & & & 500 & SON & 200 & $100 \mathrm{~N}$ & 100.0000 & 0000 \\
\hline 200325 & 10 & 100 & 1. & & 70 & ON & OON & $00 \mathrm{H}$ & 000 & 1000.0000 \\
\hline 200826 & & & & & & & OON & OOOH & 0000 & 1500.0000 \\
\hline 200827 & 3.0000 & 7.0000 & 7.0000 & 0.5000 & 1000.0000 & $0.5000 \mathrm{~N}$ & $200.0000 \mathrm{~N}$ & $10.0000 \mathrm{H}$ & 10.0000 & 1500,0000 \\
\hline 200528 & & & & & & & & & & 0000 \\
\hline 20829 & & & & & 500 & & $\mathrm{OOH}$ & OOH & 000 & 0000 \\
\hline 200830 & 5.0000 & & 7 & 0.5000 & 1000 & & 200. & OOOH & 000 & 0000 \\
\hline 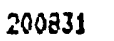 & & & & & & & & & & \\
\hline 00832 & & & & & . & & & & & 000 \\
\hline 200833 & 00 & & & & 100 & & & & & \\
\hline 200834 & 0000 & & & & & & OON & & & 0000 \\
\hline 0.075 & & & & & & & & & & 300.0000 \\
\hline 00836 & .0000 & 1.0000 & 0.7000 & 0.2000 & 500.0000 & $0.5000 \mathrm{~N}$ & OON & $10,0000 \mathrm{~N}$ & 30.0000 & 500.0000 \\
\hline & & & & & & & & $10,0000 \mathrm{H}$ & & 100.0000 \\
\hline 260838 & .0000 & & $7 \mathrm{~A}$ & & 1000.0000 & $0,5000 \mathrm{H}$ & $200,0000 \mathrm{~N}$ & $10.0000 \mathrm{H}$ & 30.0000 & 700.0000 \\
\hline 200839 & 0000 & 1.0000 & $7 m a$ & 0.2000 & 100.0000 & & & $000 \mathrm{H}$ & 70.0000 & 500.0000 \\
\hline $2 \cos 40$ & 0000 & & 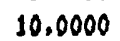 & & & & $\mathrm{OOH}$ & $00 \mathrm{H}$ & 50.0000 & 1000 \\
\hline 260841 & & & & & & & & $10.0060 \mathrm{H}$ & 70.0000 & 300.0000 \\
\hline 200842 & .0000 & 2,0000 & 5.0000 & 0.2000 & 1500.0000 & $0,5000 \mathrm{~N}$ & $200.0000 \mathrm{~N}$ & $10.0000 \mathrm{~N}$ & 30.0000 & 200.0000 \\
\hline 206843 & .0000 & 2.0000 & 20.0000 & 0.2000 & 1500,0000 & & 200,000011 & 10.000014 & 20.0000 & \\
\hline 2008 & & & & & & & & & & \\
\hline 200845 & .0000 & 7,0000 & 5.0 & 0.5000 & 1000.0000 & $0.5000 \mathrm{~N}$ & $200.0000 \mathrm{H}$ & $10.0000 \mathrm{~N}$ & 20.0000 & 150.0000 \\
\hline 260846 & 7.0000 & 10.0000 & & & 1000.0000 & & $200.0000 \mathrm{~N}$ & $10.0000 \mathrm{~N}$ & & \\
\hline 200847 & 5.0000 & 2.0060 & 1.0000 & 0.2000 & 700.0000 & $0.5000 \mathrm{~N}$ & $200.0000 \mathrm{~N}$ & $10,0000 \mathrm{~N}$ & 50.0000 & 700.0000 \\
\hline
\end{tabular}


Table 1.--Analytical data for rock chip sampling--Continued

FRINTOUT FOR SPEC/AA

\begin{tabular}{|c|c|c|c|c|c|c|c|c|c|c|}
\hline SAMFLE & S-BE & $S-B I$ & $-C D$ & $\mathrm{~s}-\mathrm{CO}$ & S-CR & $s-c u$ & $S-L A$ & $5-150$ & S-HB & S-HI \\
\hline $\begin{array}{l}200798 \\
200799\end{array}$ & $\begin{array}{l}1.00001 \\
1.0000 L\end{array}$ & $\begin{array}{l}10.0000 \mathrm{~N} \\
10.0000 \mathrm{H}\end{array}$ & $\begin{array}{l}20.0000 \mathrm{~N} \\
20.0000 \mathrm{~N}\end{array}$ & $\begin{array}{l}50.0000 \\
30.0000\end{array}$ & $\begin{array}{l}200.0000 \\
150.0000\end{array}$ & & & $\begin{array}{l}5.0000 \mathrm{~N} \\
5.0000 \mathrm{~N}\end{array}$ & $\begin{array}{l}20.0000 \mathrm{H} \\
20.0000 \mathrm{~N}\end{array}$ & 20.0000 \\
\hline 200800 & 1.00006 & $10.0000 \mathrm{~N}$ & $20.0000 \mathrm{~N}$ & $5.0000 \mathrm{~L}$ & 150.0000 & 15.0000 & & 5.000011 & $20.0000 N$ & 15.0000 \\
\hline 200801 & & & & & & & & & $0600 \mathrm{~N}$ & 100.0000 \\
\hline 200802 & $1.000 \mathrm{CL}$ & 0.600011 & $20.0000 \mathrm{~N}$ & 20.0000 & 200.0000 & 20.0000 & .0000 & $000 H$ & $20.0000 \mathrm{H}$ & 0000 \\
\hline 200803 & $1.0000 \mathrm{~L}$ & $10.0000 \mathrm{H}$ & $20.0000 \mathrm{~N}$ & $5.0000 \mathrm{~N}$ & 150.0000 & 15.0000 & .0000 & $0000 \mathrm{~N}$ & 20.000011 & 20.0000 \\
\hline 200304 & & & & & & & & & & \\
\hline 200805 & & & & 50.0000 & & & & & $00 \mathrm{OH}$ & \\
\hline 200806 & & & & & & & & & & \\
\hline $\begin{array}{l}200807 \\
200898\end{array}$ & & & & & & & & & & \\
\hline 200809 & $1.0000 L$ & 10.000014 & $20.0000 \mathrm{~N}$ & 30.0000 & 500.0000 & 70.0000 & 20.0000 & $0000 \mathrm{~N}$ & $20.0000 \mathrm{~N}$ & 20.0000 \\
\hline 200810 & & & & & & & & & & \\
\hline 200811 & & & & 000 & 000 & & & & $000 \mathrm{H}$ & \\
\hline 200612 & & & & 30.0000 & & & & 0011 & $000 \mathrm{NH}$ & .0000 \\
\hline 200813 & & $W$ & & 20.0000 & 0000 & & & & & \\
\hline 200814 & GL & & & 0.0000 & 3000 & & & & 30014 & .0000 \\
\hline 200815 & & 10.6 & & 5.0000 & & & & & & 0000 \\
\hline 200816 & & 10. & & 20.0000 & & 100 & & & & \\
\hline 200817 & & & & 50.0000 & & & & & & \\
\hline 200818 & OL & 10. & 20. & 50.0000 & 00 & & OOON & & & 70.0000 \\
\hline 200819 & & 10.6 & & 70.0000 & & & $100 \mathrm{~N}$ & & & .0000 \\
\hline 200820 & & & & 20. & & & SON & & OOH & \\
\hline 200821 & & OON & & 20. & & & OON & & & \\
\hline 0822 & & & & & & & & & & 1000 \\
\hline $092 \pi$ & & & & & & & & & & \\
\hline 0824 & & & & 90 & & & & & & \\
\hline 1825 & & & & & & & & & & \\
\hline 36 & & & & & & & & & & \\
\hline 200827 & & & & 20. & 100 & & & & & \\
\hline & & & & & & & & & & \\
\hline 20 & & & & & & & & & & \\
\hline 70uera & & & & & & & & & & \\
\hline & & & & & & & & & & \\
\hline 2005 & & & & & & & & & & \\
\hline 200333 & $1.0000 \mathrm{~N}$ & $10.0000 \mathrm{~N}$ & & 30. & 00 & 00 & OOL & OON & OON & 50.0000 \\
\hline 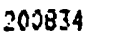 & & & & & & & & & & \\
\hline & & & & & & & & & & \\
\hline 2008 ? & & & OON & .0000 & 100 & & & & & 000 \\
\hline 200837 & & & & 50.0000 & & & & & & \\
\hline & & & & & & & & & & \\
\hline מאו & & & & 10.0000 & & & & & & \\
\hline & & & & & & & & & & \\
\hline & & & & & & & & & & \\
\hline 200842 & & $10.0000 \mathrm{H}$ & $20.0000 \mathrm{~N}$ & 10.0000 & & & & & & 50.0000 \\
\hline & & & & & & & & & & \\
\hline 2008 & & & & & 000 & & . & & OOON & 30.0000 \\
\hline 2058 & $1.6000 \mathrm{~N}$ & $10.0000 \mathrm{~N}$ & $20.0000 \mathrm{~N}$ & 50.0000 & 1000.0000 & & & & & 70.0000 \\
\hline & & & & & & & & & & \\
\hline 200847 & $1.0000 \mathrm{~N}$ & $10.0000 \mathrm{~N}$ & $20.0000 \mathrm{~N}$ & 20.0000 & 100.0000 & 5.0000 & $20.0000 \mathrm{~L}$ & 5.000014 & $20.0000 \mathrm{~N}$ & 7.0000 \\
\hline
\end{tabular}


Table 1.--Analytical data for rock chip sampling--Continued

FRINTOUT FOR SFEC/AA

\begin{tabular}{|c|c|c|c|c|c|c|c|c|c|c|}
\hline ARPLE & $S-F^{\prime} B$ & $-S B$ & $-5 C$ & $S-S N$ & S-5R & $-v$ & $5-4$ & S-Y & $-2 N$ & $5-2 R$ \\
\hline 000798 & $\begin{array}{l}10.000001 \\
: 0.00001\end{array}$ & $\begin{array}{l}100,0000 \mathrm{H} \\
100,0000 \mathrm{~N}\end{array}$ & $\begin{array}{l}50.0000 \\
50.0000\end{array}$ & $\begin{array}{l}10.0000 \mathrm{~N} \\
10.0000 \mathrm{~N}\end{array}$ & 200.0000 & $\begin{array}{l}00.0000 \\
00.0000\end{array}$ & $\begin{array}{l}0.0000 \mathrm{~N} \\
0.0000 \mathrm{~N}\end{array}$ & $\begin{array}{l}30.0000 \\
20.0000\end{array}$ & $\begin{array}{l}00.0000 \mathrm{H} \\
0.00 .0000 \mathrm{~N}\end{array}$ & \\
\hline 200800 & 10.00002 & $100.0000 \mathrm{~N}$ & 15.0000 & $10.0000 \mathrm{~N}$ & 200.0000 & 00.0000 & $0.0000 \mathrm{~N}$ & 10.0000 & $00.0000 \mathrm{~N}$ & 200.0000 \\
\hline $\begin{array}{l}200801 \\
200802\end{array}$ & $\begin{array}{l}10.0000 \mathrm{~L} \\
10.0000 \mathrm{~L}\end{array}$ & $\begin{array}{l}100.0000 \mathrm{~N} \\
100.0000 \mathrm{H}\end{array}$ & $\begin{array}{l}15.0000 \\
50.0000\end{array}$ & $\begin{array}{l}10.0000 \mathrm{~N} \\
10.0000 \mathrm{~N}\end{array}$ & $\begin{array}{l}200.0000 \\
200.0000\end{array}$ & $\begin{array}{l}150.0000 \\
100.0000\end{array}$ & $\begin{array}{l}0.0000 \mathrm{~N} \\
0.0000 \mathrm{~N}\end{array}$ & $\begin{array}{l}30.0000 \\
20.0000\end{array}$ & $\begin{array}{l}000.0000 \mathrm{~N} \\
000.0000 \mathrm{~N}\end{array}$ & $\begin{array}{l}100.0000 \\
100.0000\end{array}$ \\
\hline 200803 & 30. & 00011 & & & & & $0000 \mathrm{~N}$ & & & \\
\hline 200804 & & $\mathrm{OOON}$ & & & & & & & & \\
\hline & & & & & & & 10ON & & & \\
\hline 200806 & & Doon & & & & & 00011 & 000 & OOON & 0000 \\
\hline 200807 & & 100 & & & 500. & & & & & \\
\hline & & & & & & & & & & \\
\hline 202609 & & 200.1 & & & & & & & & \\
\hline 200810 & & $00 \mathrm{~N}$ & & & 50 & & & & & \\
\hline 0811 & & & & & & & & & & \\
\hline 0812 & & & & & & & $00 \mathrm{H}$ & & & \\
\hline דירמ & & & & & & & & & & \\
\hline 00834 & & & & & & & & & & \\
\hline 200815 & OL & OOCN & 000 & DON & 50 & & $00 \mathrm{H}$ & 000 & OOH & 000 \\
\hline 200816 & & & & & & & & & & \\
\hline 200818 & & 0011 & 000 & & & & OON & & & \\
\hline 00819 & & & & & & & DON & & & 000 \\
\hline 200820 & & & & & & & & & & 1000 \\
\hline 0821 & & & & & & & & & & \\
\hline & & & & & & & & & & \\
\hline 06 & & & & & & & & & & \\
\hline & & & & & & & & & & \\
\hline & & & & & & & & & & \\
\hline 200827 & & & & & & & & & & \\
\hline & & & & & & & & & & \\
\hline & & & & & & & & & & \\
\hline 200636 & & & & & & & & & & \\
\hline 200831 & & & & & & & & & & \\
\hline & & & & & & & & & & \\
\hline 00833 & & & & & & & ON & & SOH & 000 \\
\hline 00834 & & & & & & & & & & \\
\hline & & & & & & & & & & 0000 \\
\hline 0836 & & & & & & & & & & \\
\hline & & & & & & & & & & \\
\hline & & & & & & & & & & \\
\hline & & & & & & & & & & \\
\hline & & & & & & & & & & 100.0000 \\
\hline 200842 & & & & & & & & & & \\
\hline & & & & & & & & & & \\
\hline & & & & & & & & & & 100.0000 \\
\hline 200845 & $.0000 \mathrm{NH}$ & $00.0000 \mathrm{NH}$ & 100 & $0000 \mathrm{H}$ & 200.8 & 00 & DOON & 000 & $000 \mathrm{H}$ & 0060 \\
\hline & & 100.0 & 50. & & 150.0000 & & & & & \\
\hline & & 100.000011 & & & & & $50.0000 \mathrm{H}$ & 10.0000 & $200.0000 \mathrm{~N}$ & \\
\hline
\end{tabular}


Table 1.--Analytical data for rock chip sampling--Continued

FRIHTOUT FOR SPEC/AA

\begin{tabular}{|c|c|c|}
\hline SAMPLE & $A A-A L 1-P$ & $A Z-A G$ \\
\hline $\begin{array}{l}200798 \\
200799\end{array}$ & $\begin{array}{l}0.0500 \mathrm{~N} \\
0.0500 \mathrm{~N}\end{array}$ & $\begin{array}{l}0.1000 L \\
0.1000 L\end{array}$ \\
\hline 200800 & $0.0500 \mathrm{~N}$ & $0.1000 \mathrm{~N}$ \\
\hline 200801 & $0.0500 \mathrm{H}$ & 0.2200 \\
\hline 200602 & $0.0500 \mathrm{~N}$ & 0.1200 \\
\hline $\begin{array}{l}200803 \\
200804\end{array}$ & $\begin{array}{l}0.0500 \mathrm{~N} \\
0.0560 \mathrm{~L}\end{array}$ & $\begin{array}{l}0.7200 \\
0.10002\end{array}$ \\
\hline 200805 & 0.1600 & $0.1000 \mathrm{~N}$ \\
\hline 200806 & $0.0500 \mathrm{~L}$ & $0,1000 \mathrm{~L}$ \\
\hline $\begin{array}{l}200807 \\
200808\end{array}$ & $\begin{array}{l}0.0500 \mathrm{~N} \\
0.1200\end{array}$ & $\begin{array}{l}0.1000 \mathrm{~L} \\
0.1000 \mathrm{~N}\end{array}$ \\
\hline 200509 & $0.6500 N$ & $0.1000 L$ \\
\hline 266810 & $0.0500 \mathrm{~K}$ & $0,1000 L$ \\
\hline 200811 & 0.0600 & 0.1200 \\
\hline 200812 & $0.0500 \mathrm{~L}$ & 0.2800 \\
\hline 200513 & $0.0500 \mathrm{~L}$ & 0.10002 \\
\hline 200814 & $0,0500 \mathrm{~N}$ & $0.1000 \mathrm{~L}$ \\
\hline 200315 & $0.0500 \mathrm{~N}$ & 0.1000 \\
\hline 200816 & $0.0500 L$ & 0.3100 \\
\hline 200817 & $0.0500 \mathrm{~N}$ & 0.1000 \\
\hline 200818 & 0.0800 & 0.10002 \\
\hline 200819 & $0.0500 \mathrm{~N}$ & $0.1000 \mathrm{~L}$ \\
\hline 200820 & $0.0500 \mathrm{~N}$ & $0.1000 \mathrm{~N}$ \\
\hline 200821 & $0.0500 \mathrm{~N}$ & 0.10001 \\
\hline 200822 & $0.0500 \mathrm{~N}$ & 0.10000 \\
\hline 200823 & 0,2200 & 0.1600 \\
\hline 200824 & $0.0500 \mathrm{~N}$ & 0.10001 \\
\hline 200825 & $0.050 \mathrm{OL}$ & $0.1000 \mathrm{~L}$ \\
\hline 200826 & $0.0500 \mathrm{~N}$ & $0.1000 \mathrm{~N}$ \\
\hline 200827 & 0.0800 & $0.1000 \mathrm{~L}$ \\
\hline 200828 & $0.0500 \mathrm{~N}$ & $0.1000 \mathrm{~N}$ \\
\hline 200829 & $0.0500 \mathrm{~N}$ & $0.1000 \mathrm{H}$ \\
\hline 200330 & $0.0500 \mathrm{~N}$ & $0.5000 N$ \\
\hline 200831 & $0.0500 \mathrm{~N}$ & $0,1000 \mathrm{~N}$ \\
\hline 200832 & $0.0500 \mathrm{~N}$ & 0.10001 \\
\hline 200833 & 0.4800 & 0.5400 \\
\hline 200834 & $0.0500 \mathrm{~N}$ & 0.2700 \\
\hline 200835 & $0.0500 \mathrm{~N}$ & 0.2100 \\
\hline 200836 & $0.0500 \mathrm{~N}$ & 0.1000 \\
\hline 200837 & $0.0500 \mathrm{~N}$ & $0.1000 \mathrm{~N}$ \\
\hline 200838 & 0.1000 & 0,10006 \\
\hline 200839 & 6.6000 & 0.6800 \\
\hline 200840 & 0.1000 & 0.10001 \\
\hline 200841 & 0.0600 & 0.10006 \\
\hline 200842 & $0.0500 \mathrm{~N}$ & $0.1000 \mathrm{~N}$ \\
\hline $\begin{array}{l}200843 \\
200844\end{array}$ & $\begin{array}{l}0.0600 \\
0.0500 \mathrm{~N}\end{array}$ & $\begin{array}{l}0.1200 \\
0.5000\end{array}$ \\
\hline 266845 & $0.0500 \mathrm{~N}$ & 0.5000 \\
\hline 200846 & $0.0500 \mathrm{~N}$ & 0.5000 \\
\hline 200847 & $0.0500 \mathrm{~N}$ & 0.10006 \\
\hline
\end{tabular}


Table 1.--Analytical data for rock chip sampling--Continued

PRINTOUT FOR SPECJAA

\begin{tabular}{|c|c|c|c|c|c|c|c|c|c|c|}
\hline SAKFLE & S.FE & S-H6 & $S-C A$ & S-TI & S-Haw & S-AG & S-AS & S-All & $S-B$ & $S-B A$ \\
\hline $\begin{array}{r}200848 \\
200849\end{array}$ & $\begin{array}{l}2.0000 \\
5.0000\end{array}$ & $\begin{array}{l}2.0000 \\
3.0000\end{array}$ & $\begin{array}{l}0.5000 \\
3.0000\end{array}$ & $\begin{array}{l}0.2000 \\
0.3000\end{array}$ & $\begin{array}{r}200.0000 \\
1000.0000\end{array}$ & $\begin{array}{l}0.5000 \mathrm{~N} \\
0.5000 \mathrm{~K}\end{array}$ & $\begin{array}{l}200.0000 \mathrm{~N} \\
200.0000 \mathrm{~N}\end{array}$ & $\begin{array}{l}10.0000 \mathrm{H} \\
10.0000 \mathrm{~N}\end{array}$ & $\begin{array}{l}10.0000 \\
10.0000\end{array}$ & $\begin{array}{l}200.0000 \\
100.0000\end{array}$ \\
\hline 200850 & 5.0000 & 5.0000 & 3.0600 & 0.2000 & 1000.0000 & $0.5000 \mathrm{~N}$ & $200.0000 \mathrm{~N}$ & $10.0000 \mathrm{~N}$ & 10.0000 & 70.0000 \\
\hline 200351 & 2.0000 & 2.0000 & 5.0000 & 0.2000 & 1000.0000 & $0.5000 \mathrm{~N}$ & $200.0000 \mathrm{~N}$ & $10.0000 \mathrm{~N}$ & $10.0000 \mathrm{~L}$ & 700.0000 \\
\hline 200852 & 3.0000 & & 0.7000 & 0.2000 & 200.0000 & $0.5000 \mathrm{~N}$ & $200.0000 \mathrm{~N}$ & $10,0000 \mathrm{~N}$ & 10.0000 & 300.0000 \\
\hline 200853 & 2.0000 & 1.0000 & 0.5000 & 0.2000 & 300.0000 & $0.5000 \mathrm{~N}$ & $200.0000 \mathrm{H}$ & $10.000 \mathrm{CH}$ & 10.0000 & 300.0000 \\
\hline 200854 & 5.0000 & 2.0000 & 0.7000 & 0.2000 & 500.0000 & $0.5000 \mathrm{~N}$ & $200.0000 \mathrm{~N}$ & $10.0000 \mathrm{H}$ & $10.0000 L$ & 300.0000 \\
\hline 200855 & 5.0800 & 2.0000 & 0.7000 & 0.2000 & 500.0000 & $0.5000 \mathrm{~N}$ & $200.0000 \mathrm{~K}$ & 10.000011 & 10.0000 & 700.0000 \\
\hline 200856 & 5.0030 & 2.0000 & 0.5000 & 0.2000 & 200.0000 & $0.5000 \mathrm{H}$ & 200.000014 & 10.000011 & 10.0000 & 100.0000 \\
\hline 206857 & 5.0000 & 2.0000 & 1.0000 & 0.2000 & 500.0000 & $0.5000 \mathrm{H}$ & $200.0000 \mathrm{~N}$ & $600 \mathrm{~N}$ & & 500.0000 \\
\hline 200658 & 5.0000 & & & & & OON & DON & $\mathrm{OOH}$ & 000 & 0000 \\
\hline 200859 & 5.0000 & 2.0000 & 0.7000 & 0.2000 & 500.0000 & $0.5000 \mathrm{~N}$ & $200.0000 \mathrm{H}$ & $10.0000 \mathrm{H}$ & 20.0000 & 300.0000 \\
\hline 200860 & 7.0000 & 5.0 & 2.0000 & 0.3000 & 1000. & $0.5000 \mathrm{~N}$ & OON & $\mathrm{OOH}$ & 000 & 100.0000 \\
\hline 200881 & 7.0000 & 7.0000 & & 3000 & 1000 & & & & & \\
\hline $2008 \leq 2$ & 2.0000 & 2.0000 & 0.5000 & 0.2000 & 500 & $O O N$ & $200.0000 \mathrm{~N}$ & $00 \mathrm{OH}$ & 1000 & 200.0000 \\
\hline 200863 & 2.0000 & 1.0000 & 0.5000 & 0.2000 & 200.0000 & $0.50001 \mathrm{~N}$ & OOOON & CON & 1000 & 500.0000 \\
\hline 200864 & 5.0000 & 5.0000 & 3.0000 & 2000 & 1000.0000 & $0.5000 \mathrm{~N}$ & $300 \mathrm{~N}$ & & 0000 & 70.0000 \\
\hline 200865 & 5.0000 & 5.0 & 5.0000 & 0.3000 & 1000.0000 & $0.5000 \mathrm{~N}$ & $200.0000 \mathrm{H}$ & OON & 0000 & 0.0000 \\
\hline 200856 & 7.0000 & 5.0000 & 3.0600 & 0.3000 & 1000.0000 & $0.5000 \mathrm{~N}$ & $200.0000 \mathrm{~N}$ & OOON & 10.0000 & 100.0000 \\
\hline 200867 & 7.0000 & 7.0000 & 3.6000 & 3000 & 1500 & $0.5000 \mathrm{~N}$ & OON & DON & 1000 & 100.0000 \\
\hline 200848 & 7.0000 & 10.0000 & 5.0000 & 0.5000 & 1000.0000 & $0.5000 \mathrm{~N}$ & $200.0000 \mathrm{H}$ & 00011 & 10.0000 & 100.0000 \\
\hline 200869 & 5.0000 & & 00 & 5000 & 1000 & $100 \mathrm{~N}$ & SOON & SOH & 0000 & .0000 \\
\hline 200870 & 5.0000 & & & 2000 & 700 & $0.5000 \mathrm{H}$ & $200.0000 \mathrm{~N}$ & OOH & 2000 & 700.0000 \\
\hline 200871 & 5.0000 & 10.0 & 5.0000 & 0.5000 & 1000.0000 & $0.5000 \mathrm{~N}$ & $200.0000 \mathrm{~N}$ & $100 \mathrm{~N}$ & 0000 & 150.0000 \\
\hline 200872 & 5.00 & & & 300 & 100 & & & & & 0000 \\
\hline 200873 & & & & 300 & & OON & & & 000 & 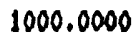 \\
\hline $\begin{array}{l}200874 \\
200875\end{array}$ & 5.0000 & 5.0 & & $\begin{array}{l}00 \\
00\end{array}$ & & & & & & \\
\hline 200876 & & & & 2000 & 500.0000 & & $200.0000 \mathrm{H}$ & & 0000 & 200.0000 \\
\hline $\begin{array}{l}200877 \\
200878\end{array}$ & $\begin{array}{l}5.0000 \\
5.0000\end{array}$ & 5.00 & & & & & & & & \\
\hline 260879 & 200voro & & & 2000 & $500.0000^{\circ}$ & $0.5000 \mathrm{~N}$ & $200.0000 \mathrm{~N}$ & & 50.0000 & 300.0000 \\
\hline $\begin{array}{l}200880 \\
200881\end{array}$ & 2.0000 & 1.0000 & & $\begin{array}{l}0.2000 \\
0.2000\end{array}$ & & & $\begin{array}{l}200.0000 \mathrm{H} \\
200.000011\end{array}$ & & & \\
\hline 200882 & 7.0060 & & & .0070 & & $0.5000 \mathrm{~N}$ & $200.0000 \mathrm{H}$ & $10.0000 \mathrm{H}$ & 10.0000 & 70.0000 \\
\hline 200883 & 10.0000 & 7.0000 & 5.0000 & 1.0000 & 1500.0000 & $0.5000 \mathrm{~N}$ & 200.000011 & 10.000011 & 50.0000 & 200.0000 \\
\hline 200884 & 10.0000 & 7.0000 & 5.0000 & 1.0000 & 2000.0000 & $0.5000 \mathrm{~N}$ & $200.0000 \mathrm{~N}$ & $10.0000 \mathrm{~N}$ & 10.0000 & 300.0000 \\
\hline 200885 & & 1.0000 & 1.0000 & 2000 & 500.0000 & $0.5000 \mathrm{~N}$ & $00 \mathrm{~N}$ & OOON & 10.0000 & 500.0000 \\
\hline 200886 & 7.0000 & 0.7000 & 5.0000 & 0.3000 & 500.0000 & $0.5000 \mathrm{~N}$ & $200.0000 \mathrm{~N}$ & 10.000011 & 10.0000 & 100.0000 \\
\hline 200887 & & & & 3000 & 100 & & & & & 100.0000 \\
\hline 200888 & & & & 000 & & DOH & & & 000 & 000 \\
\hline 200889 & & 2.0000 & & & & & & & & \\
\hline 200990 & & 10. & & & 150 & & OHI & & & 1000 \\
\hline & & & & & & & & & & \\
\hline 200892 & 7,0000 & & 5.0 & 1000 & 1500.0000 & $0.5000 \mathrm{~N}$ & $\mathrm{OOH}$ & DON & 000 & 500.0000 \\
\hline 200893 & & & & & & & & & 000 & 700,0000 \\
\hline & & & & & & & & & & \\
\hline 200895 & 3.0000 & & 1.0000 & 0.2000 & 200.0000 & $0.5000 \mathrm{NH}$ & 200.000011 & $10.0000 \mathrm{~N}$ & 70.0000 & 500.0000 \\
\hline 2008 & & & 1.0000 & & 500 & 0.5 & 200 & $10.0000 \mathrm{~N}$ & 50.0000 & \\
\hline 200897 & 3.0000 & 0,3000 & 1.0000 & 0.2000 & 500.0000 & $0.5000 \mathrm{~N}$ & $200.0000 \mathrm{H}$ & $10.0000 \mathrm{~N}$ & 50.0000 & 700.0000 \\
\hline
\end{tabular}


Table 1.--Analytical data for rock chip sampling--Continued

PRINTOUT FOR SPECJAA

\begin{tabular}{|c|c|c|c|c|c|c|c|c|c|c|}
\hline SAKFLE & $-B E$ & $S-8 I$ & $S-C D$ & $-C O$ & S-CK & $s-c u$ & $S-L A$ & -100 & $-N B$ & -HI \\
\hline $\begin{array}{l}200848 \\
200849\end{array}$ & $\begin{array}{l}1.0000 \mathrm{~N} \\
1.0000 \mathrm{~N}\end{array}$ & $\begin{array}{l}10.0000 \mathrm{~N} \\
10.0000 \mathrm{H}\end{array}$ & $\begin{array}{l}20.0000 \mathrm{~N} \\
20.0000 \mathrm{~N}\end{array}$ & $\begin{array}{l}10.00000 \\
50.0000\end{array}$ & $\begin{array}{l}150.0000 \\
200.0000\end{array}$ & $\begin{array}{l}20.0000 \\
30.0000\end{array}$ & $\begin{array}{l}20.0000 L \\
20.0000 L\end{array}$ & $\begin{array}{l}5.0000 \mathrm{~N} \\
5.0000 \mathrm{NH}\end{array}$ & $\begin{array}{l}20.0000 \mathrm{~N} \\
20.0000 \mathrm{~N}\end{array}$ & $\begin{array}{l}50.0000 \\
50.0000\end{array}$ \\
\hline 200850 & 1.000011 & 10.000011 & $20.0000 \mathrm{~N}$ & 50.0000 & 200.0000 & 50.0000 & $20.0000 \mathrm{~L}$ & $5.0000 \mathrm{~N}$ & $20.0000 \mathrm{~N}$ & 70.0000 \\
\hline 200851 & $1.0600 \mathrm{~N}$ & 10.000014 & 20.000011 & & & & & $5.0000 \mathrm{H}$ & $0.0000 \mathrm{~N}$ & 50.0000 \\
\hline 00852 & $1.0050 \mathrm{~N}$ & .000011 & ION & & & & & & & \\
\hline 200853 & $1.000 \mathrm{CH}$ & $10.0000 \mathrm{~N}$ & $20.000 \mathrm{ON}$ & 20.0000 & 150.0000 & 5.0000 & 20.0000 & $5.0000 \mathrm{~N}$ & $0.0000 \mathrm{H}$ & 50.0000 \\
\hline $\begin{array}{l}200854 \\
200855\end{array}$ & & & & & & & & & & \\
\hline 200856 & $1.0000 \mathrm{~N}$ & $10.0000 \mathrm{H}$ & $20.0000 \mathrm{~N}$ & 20.0000 & 150.0000 & & & & & 50.0000 \\
\hline 008 & & & & & & & & & & \\
\hline 000858 & $1,0000 \mathrm{~N}$ & $10,0000 \mathrm{~N}$ & $0.0000 \mathrm{H}$ & & 200 & & & & & 0000 \\
\hline 200859 & $1.0000 \mathrm{~N}$ & 10.000011 & $20.0000 \mathrm{~N}$ & 20.0000 & 150 & & & & & \\
\hline 00580 & & & & & & & & & & 0.0000 \\
\hline 200861 & 1.000011 & 10. & & & & & & $\mathrm{OOOH}$ & OOH & .0000 \\
\hline 200882 & $1.0000 \mathrm{~N}$ & 10.0 & 20.000011 & 20.0000 & 200 & & & OOH & OON & 0000 \\
\hline 200863 & $1.0000 \mathrm{H}$ & 10. & $20.0000 \mathrm{~N}$ & 20.0000 & 150.0000 & & & 5.000011 & OOON & 0000 \\
\hline 2008 & & & & & & & & OOOH & MON & 0000 \\
\hline 200865 & 1.0000011 & OOH & & 50 & 200 & & & $100 \mathrm{~N}$ & & .0000 \\
\hline $\begin{array}{l}200866 \\
200867\end{array}$ & & & & & & & & & & \\
\hline 200868 & $1.0000 \mathrm{~L}$ & $10.0000 \mathrm{~N}$ & $20.0000 \mathrm{~N}$ & 30.0000 & 200.0000 & 000 & & $5.0000 \mathrm{~N}$ & OOON & 50.0000 \\
\hline 200869 & $1.0000 \mathrm{~L}$ & 10. & & 50 & 200 & & & OON & OON & 0000 \\
\hline 200870 & 1.00001 & 10.0 & OHI & 10. & 100. & 001 & & DON & $\mathrm{OOH}$ & .00000 \\
\hline 10871 & 1.00002 & $10.0000 \mathrm{H}$ & OON & & & & & OON & OOON & 50.0000 \\
\hline 0872 & OOL & 0011 & & 50. & & & & OON & OON & 50.0000 \\
\hline 200873 & .00001 & & $O N$ & 30. & & & & & & 0000 \\
\hline 300874 & SOMOOI & & 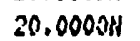 & & & & & & & 50.0000 \\
\hline 108 & & & & & & & & & & 000 \\
\hline 2608 & & & & so & & & & & & 000 \\
\hline ריפחטי & 1.00001 & & & & 150 & & & & & 70.0000 \\
\hline 00978 & & & & & & & & & & 000 \\
\hline 260879 & $1.0000 \mathrm{~L}$ & & & & 100 & & & & & 000 \\
\hline 200880 & $1,000 \mathrm{CL}$ & & $\mathrm{OON}$ & 5.0000 & 150 & & & & & 000 \\
\hline 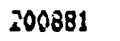 & I & & & $5.0000 \mathrm{~N}$ & & & & & & 10.0000 \\
\hline $20088 ?$ & & & & $.0000 \mathrm{~N}$ & 100 & & & $00 \mathrm{H}$ & OON & .0000 \\
\hline 200883 & $1.0000 \mathrm{~L}$ & OOH & ONH & 70. & 100 & 000 & $O L$ & OOH & OON & 0000 \\
\hline 200884 & $1.0000 \mathrm{~L}$ & ONI & ONH & 70. & 150. & 100 & & $5.0000 \mathrm{~N}$ & $\mathrm{OH}$ & 000 \\
\hline 200885 & $1.0000 \mathrm{~L}$ & .000014 & & & 350. & & & OOOH & & 000 \\
\hline 200996 & $1.0000 \mathrm{~L}$ & $10.0000 \mathrm{~K}$ & $20.0000 \mathrm{~N}$ & 50.0000 & 200.0 & & & $5.0000 \mathrm{~K}$ & & 0000 \\
\hline & & & & & & & & & & 70.0000 \\
\hline 6888 & 00008 & ON & & & 150 & & & 5.0000 & & 10.0000 \\
\hline 200889 & $1.0000 L$ & & & & 200 & & & & & 5.0000 \\
\hline 20085 & & & & & & & & & & \\
\hline & & & & & & & & & & \\
\hline 20089 & $\alpha L$ & & & & 300. & & & & & 50.0000 \\
\hline 200893 & f & & & & & & & & & 70.0000 \\
\hline & & & & & & & & & & \\
\hline & & & & & & & & & & 10.0000 \\
\hline 8896 & 1.00001 & $\begin{array}{l}10.0000 \mathrm{~N} \\
10.00001\end{array}$ & $\begin{array}{l}20.0000 \mathrm{H} \\
20.0000 \mathrm{~N}\end{array}$ & $\begin{array}{l}5.0000 \\
5.0000\end{array}$ & $\begin{array}{l}500,00000 \\
150,0000\end{array}$ & $\begin{array}{l}50.0000 \\
20.0000\end{array}$ & 0.0000 & $\begin{array}{l}20.0000 \\
20.0000\end{array}$ & $\begin{array}{l}20.0000 \mathrm{~N} \\
20.0000 \mathrm{~N}\end{array}$ & $\begin{array}{l}10.0000 \\
10.0000\end{array}$ \\
\hline & & & & & & & & & & \\
\hline
\end{tabular}


Table 1.--Analytical data for rock chip sampling--Continued

PRINTOUT FOR SPEC/AA

\begin{tabular}{|c|c|c|c|c|c|c|c|c|c|c|}
\hline SALPLE & $S-P B$ & S-SB & S-SC & S-SH & $-5 R$ & $s-v$ & $s-w$ & S-Y & $S-Z M$ & $-2 R$ \\
\hline $\begin{array}{l}00848 \\
00849\end{array}$ & $\begin{array}{l}10.0000 \mathrm{~N} \\
10.0000 \mathrm{~N}\end{array}$ & $\begin{array}{l}100.0000 \mathrm{~N} \\
100.0000 \mathrm{H}\end{array}$ & $\begin{array}{l}20.0000 \\
20.0000\end{array}$ & $\begin{array}{l}10.0000 \mathrm{~N} \\
10.0000 \mathrm{H}\end{array}$ & $\begin{array}{l}200.0000 \\
200.0000\end{array}$ & $\begin{array}{r}50.0000 \\
300.0000\end{array}$ & $\begin{array}{l}50.0000 \mathrm{~N} \\
50.0000 \mathrm{~N}\end{array}$ & $\begin{array}{l}10.0000 \\
20.0000\end{array}$ & $\begin{array}{l}200.0000 \mathrm{~N} \\
200.0000 \mathrm{~N}\end{array}$ & $\begin{array}{l}100,0000 \\
100.0000\end{array}$ \\
\hline 00850 & $10.0000 \mathrm{H}$ & $100.0000 \mathrm{H}$ & 10.0000 & $10.0000 \mathrm{~N}$ & 200.0000 & 300.0000 & $.0000 \mathrm{H}$ & 0000 & $0000 \%$ & 70.0000 \\
\hline 00851 & $10.0000 \mathrm{~N}$ & $100.000 \mathrm{CH}$ & .0000 & 10.000011 & 500.0000 & 100.0000 & $.0000 \mathrm{H}$ & 20.0000 & $200.0000 \mathrm{~N}$ & 150.0000 \\
\hline 00852 & $10.0000 \mathrm{~N}$ & $100.0000 \mathrm{~N}$ & .0000 & $10.0000 \mathrm{~W}$ & 300.0000 & 50.0000 & $.0000 \mathrm{~N}$ & 0.0000 & $00.0000 \mathrm{~N}$ & 100.0000 \\
\hline 60853 & $10.0000 \mathrm{H}$ & 100 & 10.0000 & 10.000011 & 200.0000 & 50.0000 & OOOOH & 0000 & $000 \mathrm{~N}$ & 200.0000 \\
\hline 0854 & $10.0000 \mathrm{H}$ & OCON & & OOON & & & $000 \mathrm{NH}$ & & 00011 & .0000 \\
\hline 0855 & & & 90 & & 200. & 100, & & & & 150,0000 \\
\hline 00856 & $10.0000 \mathrm{~N}$ & $100.0000 \mathrm{~N}$ & & $10.0000 \mathrm{~N}$ & 150.0000 & 70.0000 & OOON & 000 & $000 \mathrm{~N}$ & 100.0000 \\
\hline 0.0857 & & & & & & & & & & \\
\hline 0859 & OON & $100.0000 \mathrm{~N}$ & 3.0000 & OON & 150 & 100. & & & OOH & 0000 \\
\hline $\begin{array}{l}0860 \\
0861\end{array}$ & & & & & & & & & OOH & \\
\hline 0862 & & ON & 00 & $100 \mathrm{~N}$ & 200 & 100,0000 & & & OOH & 200.0000 \\
\hline & & & & & & & & & & \\
\hline 0860 & & & & & & & & & & 0000 \\
\hline 55 & & 100 & & OON & & & & & OON & 0000 \\
\hline & & & & DON & & & & & $\mathrm{OOH}$ & 0000 \\
\hline 7 & & & & & & & & & OON & 0000 \\
\hline $08<8$ & & & & & & & & & $100 \mathrm{H}$ & 100.0000 \\
\hline 0869 & & ONH & & & & & & & $\mathrm{OOH}$ & 000 \\
\hline 0870 & & ON & & & & & ON & & OOH & 0000 \\
\hline 371 & & & & & & & & & OON & 000 \\
\hline 0872 & & & & & & & & & & \\
\hline & & & & & & & & & & \\
\hline & & & & & & & & & & \\
\hline & & & & & & & & & OOH & 0000 \\
\hline 0827 & & & & & & & & & & \\
\hline 09 & & & & & & & & & & 000 \\
\hline & & & & & & & & & & \\
\hline & & & & & & & & & & \\
\hline & & & & & & & & & & \\
\hline & & & & & 6ON & & & & OON & $0000 \mathrm{~N}$ \\
\hline 0883 & & & & OON & & & & & OON & \\
\hline & & & & & & & & & & \\
\hline & & & & & & & & & & \\
\hline 0886 & & & & & & & & & OON & 0000 \\
\hline 0808 & & & & & & & & & & 1000 \\
\hline & & & & & & & & & & \\
\hline & & & & & & & & & & \\
\hline & & & & & & & & & & \\
\hline & & & & & & & & & 10ON & 150.0000 \\
\hline 200892 & & 100. & & $100 \mathrm{~N}$ & & & & & & 100.0000 \\
\hline & & & & & & & & & & \\
\hline & & & & & & & & & & \\
\hline & & & & & & & & & & \\
\hline & & & & & & & & & & \\
\hline & & & $5.0000 \mathrm{~L}$ & $10.0000 \mathrm{H}$ & 200.0000 & 50.0000 & SOH & 15.0000 & 20 & 200.000 \\
\hline
\end{tabular}


Table 1.--Analytical data for rock chip sampling--Continued

FRINTOUT FOR SPECVAA

\begin{tabular}{|c|c|c|}
\hline SAKFLE & $A A-A U-F$ & $A Z-A G$ \\
\hline $\begin{array}{l}200848 \\
200849\end{array}$ & $\begin{array}{l}0.0500 \mathrm{~N} \\
0.0500 \mathrm{~N}\end{array}$ & $\begin{array}{l}0.1000 \mathrm{~N} \\
0.1000 \mathrm{~N}\end{array}$ \\
\hline 200850 & $0.0500 \mathrm{H}$ & 0.1500 \\
\hline 200851 & $0,0500 \mathrm{~N}$ & $0.1000 \mathrm{H}$ \\
\hline 200852 & $0.0500 \mathrm{~N}$ & $0.1000 \mathrm{~N}$ \\
\hline 200853 & $0,0500 \mathrm{~N}$ & $0.1000 \mathrm{~N}$ \\
\hline 200854 & $0.0500 \mathrm{~N}$ & $0.1000 \mathrm{~N}$ \\
\hline 200855 & $0.0500 \mathrm{~N}$ & $0.1000 \mathrm{~N}$ \\
\hline 200856 & $0.0500 \mathrm{~L}$ & $0,1000 \mathrm{N1}$ \\
\hline 200857 & 0.0800 & $0,1000 \mathrm{~N}$ \\
\hline 200358 & 0.0500 & $0.1000 \mathrm{~N}$ \\
\hline 200859 & $0.0500 \mathrm{~N}$ & $0.1000 \mathrm{H}$ \\
\hline 200860 & $0,0500 \mathrm{~N}$ & $0.1000 \mathrm{~N}$ \\
\hline 200861 & $0.0500 \mathrm{~N}$ & $0,1000 L$ \\
\hline 290862 & $0.0500 \mathrm{~N}$ & $0.1000 \mathrm{~N}$ \\
\hline 200863 & $0.0500 \mathrm{~N}$ & $0.1000 \mathrm{~N}$ \\
\hline 200864 & $0.0500 \mathrm{~N}$ & $0.1000 \mathrm{~N}$ \\
\hline 200865 & 0.2700 & 0.2000 \\
\hline $\begin{array}{l}200866 \\
200867\end{array}$ & $\begin{array}{l}0.0500 \mathrm{~N} \\
0.0500 \mathrm{~N}\end{array}$ & $\begin{array}{l}0.1000 L \\
0.1000 L\end{array}$ \\
\hline 200868 & $0.0500 \mathrm{~N}$ & $0.1000 \mathrm{~N}$ \\
\hline 200869 & $0.0500 \mathrm{H}$ & $0.1000 \mathrm{~N}$ \\
\hline 200870 & 0.050011 & $0.1000 \mathrm{~N}$ \\
\hline 200871 & 0.050011 & $0.1000 \mathrm{~N}$ \\
\hline $\begin{array}{l}200872 \\
200873\end{array}$ & $\begin{array}{l}0.0500 \mathrm{~N} \\
0.0500 \mathrm{~N}\end{array}$ & $\begin{array}{l}0.1000 \mathrm{~L} \\
0.1000 \mathrm{~N}\end{array}$ \\
\hline 200874 & $0.0500 \mathrm{~N}$ & $0.1000 \mathrm{~N}$ \\
\hline $\begin{array}{l}200675 \\
200876\end{array}$ & $\begin{array}{l}0.0500 \mathrm{~N} \\
0.0500 \mathrm{~N}\end{array}$ & $\begin{array}{l}0.1000 \mathrm{~N} \\
0.1000 \mathrm{~N}\end{array}$ \\
\hline 200877 & $0.0500 \mathrm{~N}$ & 0.1000 \\
\hline 200878 & $0.0500 \mathrm{~N}$ & $0.1000 \mathrm{~N}$ \\
\hline 200879 & $0.0500 \mathrm{~N}$ & 0,2000 \\
\hline 200880 & $0.0500 \mathrm{~N}$ & $0.1000 \mathrm{~N}$ \\
\hline 200881 & $0.0500 \mathrm{~N}$ & 0.1500 \\
\hline 200882 & $0.0500 \mathrm{~N}$ & $0.1000 \mathrm{~N}$ \\
\hline 200883 & $0.0500 \mathrm{~N}$ & $0.1000 \mathrm{~L}$ \\
\hline 200884 & $0.0500 \mathrm{~N}$ & $0.1000 \mathrm{~N}$ \\
\hline 200885 & $0.0500 \mathrm{~N}$ & $0.1000 \mathrm{~N}$ \\
\hline 200886 & $0.0500 \mathrm{~N}$ & $0.1000 \mathrm{H}$ \\
\hline $20 c 887$ & $0.0500 \mathrm{~N}$ & $0.1000 \mathrm{~N}$ \\
\hline 200888 & $0.0500 \mathrm{H}$ & $0,1000 \mathrm{~N}$ \\
\hline 200889 & $0.0500 \mathrm{~N}$ & $0.1000 \mathrm{~N}$ \\
\hline 200850 & $0.0500 \mathrm{~N}$ & $0.1000 \mathrm{~N}$ \\
\hline $\begin{array}{l}200891 \\
200892\end{array}$ & $\begin{array}{l}0.0500 \mathrm{~N} \\
0.0500 \mathrm{~N}\end{array}$ & $\begin{array}{l}0.1000 \mathrm{H} \\
0.1000 \mathrm{~N}\end{array}$ \\
\hline 200893 & $0.0500 \mathrm{~N}$ & $0,1000 \mathrm{~N}$ \\
\hline 200894 & $0.0500 \mathrm{~N}$ & $0,1000 \mathrm{~N}$ \\
\hline 200895 & 0.05001 & $0.1000 \mathrm{~N}$ \\
\hline 200896 & $0.0500 \mathrm{~N}$ & $0.1000 \mathrm{H}$ \\
\hline 200897 & $0.0500 \mathrm{~N}$ & $0.1000 \mathrm{~N}$ \\
\hline
\end{tabular}


Table 1.--Analytical data for rock chip sampling--Continued

PRINTOUT FOR SPEC/AA

\begin{tabular}{|c|c|c|c|c|c|c|c|c|c|c|}
\hline SAMPLE & S-FE & S-HG & $S-C A$ & S-TI & S-HAN & S-AG & S-AS & S-ÂU & $5-8$ & $S-B A$ \\
\hline $\begin{array}{l}200899 \\
200899\end{array}$ & $\begin{array}{l}5.0000 \\
5.0000\end{array}$ & $\begin{array}{l}7.0000 \\
5.0000\end{array}$ & $\begin{array}{l}3.0000 \\
2.0000\end{array}$ & $\begin{array}{l}0.3000 \\
0.3000\end{array}$ & $\begin{array}{l}700.0000 \\
500.0000\end{array}$ & $\begin{array}{l}0.5000 \mathrm{H} \\
0.5000 \mathrm{H}\end{array}$ & $\begin{array}{l}200.0000 \mathrm{~N} \\
200.0000 \mathrm{~N}\end{array}$ & $\begin{array}{l}10.0000 \mathrm{~N} \\
10.0000 \mathrm{~N}\end{array}$ & $\begin{array}{l}20.0000 \\
20.0000\end{array}$ & $\begin{array}{l}700.0000 \\
300.0000\end{array}$ \\
\hline 200900 & 5.0000 & 2.0000 & 3.0000 & 0.2000 & 1000,0000 & $0.5 \mathrm{POOH}$ & $200.0000 \mathrm{~N}$ & $10.0000 \mathrm{~N}$ & 30.0000 & 1000.0000 \\
\hline $\begin{array}{l}200901 \\
200902\end{array}$ & $\begin{array}{l}5.0000 \\
5.0000\end{array}$ & $\begin{array}{l}1.0000 \\
1.0000\end{array}$ & $\begin{array}{l}2.0000 \\
2.0000\end{array}$ & $\begin{array}{l}0.2000 \\
0.5000\end{array}$ & $\begin{array}{r}500,0000 \\
1000,0000\end{array}$ & $\begin{array}{l}0.5000 \mathrm{H} \\
0.5000 \mathrm{HH}\end{array}$ & $\begin{array}{l}200.0000 \mathrm{~N} \\
200.0000 \mathrm{H}\end{array}$ & $\begin{array}{l}10.0000 \mathrm{~N} \\
10.0000 \mathrm{~N}\end{array}$ & $\begin{array}{l}50.0000 \\
30.0000\end{array}$ & $\begin{array}{r}700.0000 \\
1500.0000\end{array}$ \\
\hline 200903 & 7.0000 & 5.0000 & 2.0000 & 1.0000 & 1000.0000 & $0.5000 \mathrm{~N}$ & $200,0000 \mathrm{~N}$ & $10.0000 \mathrm{~N}$ & 0.00001 & 500.0000 \\
\hline $\begin{array}{l}200904 \\
200905\end{array}$ & $\begin{array}{l}5.0000 \\
5.0000\end{array}$ & $\begin{array}{l}5.0000 \\
2.0000\end{array}$ & $\begin{array}{r}2.0000 \\
10.0000\end{array}$ & $\begin{array}{l}0.2000 \\
0.2000\end{array}$ & $\begin{array}{r}500,0000 \\
1500.0000\end{array}$ & $\begin{array}{l}0.5000 \mathrm{~N} \\
0.5000 \mathrm{~N}\end{array}$ & $\begin{array}{l}200,0000 \mathrm{~N} \\
200,0000 \mathrm{~N}\end{array}$ & $\begin{array}{l}10.0000 \mathrm{~N} \\
10.0000 \mathrm{~N}\end{array}$ & $\begin{array}{l}20.0000 \\
10.0000\end{array}$ & $\begin{array}{r}1000.0000 \\
200.0000\end{array}$ \\
\hline 200906 & 5.0000 & 2.0000 & 1.5000 & 0.2000 & 700.0000 & $0.5000 \mathrm{~N}$ & $200.0000 \mathrm{~N}$ & 10.000011 & 15.0000 & 1000,0000 \\
\hline 200907 & 16.0000 & 10.0000 & 7.0000 & 1.0000 & 200.0000 & $0.5000 \mathrm{~N}$ & $200.0000 \mathrm{~N}$ & $10.0000 \mathrm{~N}$ & .0000 & 300.0000 \\
\hline 200908 & & & 20.0000 & 0.2000 & 2000 & OON & & & $000 \mathrm{~L}$ & .0000 \\
\hline 200909 & 5.0000 & 2.0000 & 1.0000 & 0.3000 & 500.0000 & $5000 \mathrm{~N}$ & OON & $0.0000 \mathrm{H}$ & .0000 & 700.0000 \\
\hline 200810 & 3.0000 & 1,5000 & 1.0000 & 0.2000 & 500.0000 & $.5000 \mathrm{~N}$ & 200.8 & $.0000 \mathrm{~N}$ & .0000 & 700.0000 \\
\hline 200911 & & & & & 500 & $\mathrm{OON}$ & & & 0000 & .0000 \\
\hline 200912 & 5.6000 & 10.0000 & 15.0000 & 0.5000 & 1000.0000 & $0.5000 \mathrm{~N}$ & $200.0000 \mathrm{~N}$ & DOON & .0000 & 500.0000 \\
\hline 200913 & & & & 0.2000 & 1000 & OOON & & OOH & 0000 & 0000 \\
\hline 200914 & & & & & & & & & & \\
\hline 200915 & 5.0000 & 10.0000 & 10.0 & 0.3000 & 1000.0000 & 100\% & 200. & $200 \mathrm{~N}$ & .0000 & 500.0000 \\
\hline 200916 & 7.0060 & 10.0000 & 10.0000 & 0.2000 & 1500.0000 & $0.5000 \mathrm{~N}$ & $200.0000 \mathrm{~N}$ & $10,0000 \mathrm{H}$ & 0.0000 & 500.0000 \\
\hline 200987 & .0000 & 00 & 1.0 & 0.2000 & 1000.0000 & OON & 200 & OON & 000 & 500.0000 \\
\hline 200918 & 5.0000 & 000 & 1.0000 & 0.3000 & 500.0000 & $100 \mathrm{~N}$ & 200 & $300 \mathrm{~N}$ & 0000 & 700.0000 \\
\hline 200919 & 0 & & & & 500. & OON & & 00011 & 0000 & .0000 \\
\hline 200920 & 0 & 7.0000 & 1.0 & 0.3000 & 1000 & $100 \mathrm{~N}$ & & OON & 000 & 1000 \\
\hline 200921 & .0000 & 7. & 3.0000 & 0.5000 & 3000,0000 & $0.5000 \mathrm{H}$ & $200.0000 \mathrm{~N}$ & OOON & 20.0000 & 700.0000 \\
\hline 200922 & & 1.0000 & 1.0000 & .2000 & 500.0000 & $0.5000 \mathrm{H}$ & 2000.0000 & $0000 \mathrm{~N}$ &., 0000 & 700.0000 \\
\hline 200923 & 0 & 5.0000 & 1.0000 & 0.3000 & 500.0000 & $0.5000 \mathrm{~N}$ & $200.0000 \mathrm{~N}$ & $10.0000 \mathrm{H}$ & 20.0000 & 1000.0000 \\
\hline 200924 & 0000 & 5.0000 & 1.0000 & 0.3000 & 1000.0000 & $5000 \mathrm{~N}$ & & $1000 \mathrm{KH}$ & .0000 & 500.0000 \\
\hline 200925 & & & & & & & & & & 1000 \\
\hline 200926 & 000 & 00 & & 00 & $\infty$ & OON & & 00014 & .0000 & 2000.0000 \\
\hline 200927 & 800 & 7.0000 & 1000 & 0.5000 & 1000,0000 & 0.500011 & & $10.0000 \mathrm{~N}$ & 30.0000 & 700.0000 \\
\hline 200928 & & & & & 100 & & & & & \\
\hline 200929 & 100 & 00 & & & 00 & OON & & OONH & 0000 & 0000 \\
\hline 200930 & 5.0000 & 5.0000 & .0000 & 0.2000 & 2000.00000 & $0.5000 \mathrm{HH}$ & 200 & $000 \mathrm{~N}$ & .0000 & 200.0000 \\
\hline 200931 & & & & & & & & & & \\
\hline 200932 & & & & & 200 & & & & 100 & 1000 \\
\hline 200933 & $\infty 0$ & 00 & .0000 & 100 & 2000 & COON & 200. & $000 \mathrm{~N}$ & .0000 & 300.0000 \\
\hline 200934 & & & & & 2000 & $0.5000 \mathrm{~N}$ & 200 & $200 \mathrm{NH}$ & 0000 & 700,0000 \\
\hline 200935 & 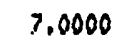 & & & & 2000 & $5000 \mathrm{~N}$ & & OON & 000 & .0000 \\
\hline 200935 & .0000 & 0000 & 15 & 0.5000 & 1500 & & & & 000 & 150.0000 \\
\hline דרפט & & & & & & & & & & \\
\hline & & & & & 300 & OON & OON & $00 \% 1$ & 0000 & 500.0000 \\
\hline 200939 & 5.0000 & 7,0000 & 1,0000 & 0.2000 & 1000. & $0,5000 \mathrm{~N}$ & $200.0000 \mathrm{~N}$ & $000 \mathrm{~N}$ & 20.0000 & 500.0000 \\
\hline 200910 & & & & & & & $200.0000 \mathrm{~N}$ & & 30.0000 & 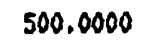 \\
\hline 200798 & & & & & & & & & 000 & 200.0600 \\
\hline 200942 & 5.0000 & 5,0000 & 3,0000 & 0.2000 & 1000.0000 & $0.5000 \mathrm{H}$ & $200.0000 \mathrm{~N}$ & $50.0000 \mathrm{~N}$ & 20.0000 & 500.0000 \\
\hline & & & & & 1000. & $0.5000 \mathrm{~N}$ & & & & 300.0000 \\
\hline & & & & & & & & & & 2000000 \\
\hline 2007 & & & & & 500.0000 & $0.5000 \mathrm{~N}$ & $200.0000 \mathrm{~N}$ & $10.0000 \mathrm{~N}$ & 30.0000 & 700.0000 \\
\hline $\begin{array}{l}200946 \\
200947\end{array}$ & $\begin{array}{l}5.0000 \\
5.6000\end{array}$ & $\begin{array}{l}7.0000 \\
5.0000\end{array}$ & $\begin{array}{r}1.0000 \\
16.0000\end{array}$ & $\begin{array}{l}0.2000 \\
0.2000\end{array}$ & $\begin{array}{l}500.0000 \\
200.0000\end{array}$ & $\begin{array}{l}0.5000 \mathrm{~N} \\
0.5000 \mathrm{~N}\end{array}$ & $\begin{array}{l}200.0000 \mathrm{~N} \\
200.0000 \mathrm{H}\end{array}$ & $\begin{array}{l}10.0000 \mathrm{~N} \\
10.0000 \mathrm{~N}\end{array}$ & $\begin{array}{l}30.0000 \\
20.0000\end{array}$ & $\begin{array}{l}500.0000 \\
700,0000\end{array}$ \\
\hline
\end{tabular}


Table 1.--Analytical data for rock chip sampling--Continued

FRINTOUT FOR SPEC/AA

\begin{tabular}{|c|c|c|c|c|c|c|c|c|c|c|}
\hline MFLE & RE & $-B I$ & $-c n$ & $-\mathrm{CO}$ & $C R$ & $c u$ & -LA & -150 & $-H B$ & -NI \\
\hline $\begin{array}{l}200898 \\
204899\end{array}$ & $\begin{array}{l}1.00001 \\
1.00001\end{array}$ & $\begin{array}{l}10.000011 \\
10.000011\end{array}$ & $\begin{array}{l}20.0000 \mathrm{~N} \\
20.000 \mathrm{CN}\end{array}$ & $\begin{array}{l}30.0000 \\
20.0000\end{array}$ & $\begin{array}{l}200.0000 \\
500.0000\end{array}$ & $\begin{array}{l}50.0000 \\
50.0000\end{array}$ & $\begin{array}{l}20,0000 \\
30,0000\end{array}$ & $\begin{array}{c}5.0000 \mathrm{~N} \\
20.0000\end{array}$ & $\begin{array}{l}20.0000 \mathrm{~N} \\
20.0000 \mathrm{H}\end{array}$ & $\begin{array}{r}100.0000 \\
70.0000\end{array}$ \\
\hline 200900 & 1.00001 & $10.0000 \mathrm{~N}$ & $20.0000 \mathrm{NH}$ & 20.0000 & 700.0000 & 0.0000 & 20.0000 & 30.0000 & 20.0000 & 70.0000 \\
\hline 200901 & $1.0000 \mathrm{~L}$ & $10.0000 \mathrm{H}$ & $0.0000 \mathrm{H}$ & 0.0000 & 200.0000 & 1000 & & 0 & OOH & 20.0000 \\
\hline 200902 & 1.00002 & $10.0000 \mathrm{~N}$ & $20.0000 \mathrm{~N}$ & .0000 & 50,0000 & .0000 & 100.0000 & $5.0060 \mathrm{H}$ & 30.0000 & $5.0000 \mathrm{H}$ \\
\hline 200903 & 1.00001 & & & & & & & & $20.0000 \mathrm{~N}$ & 70.0000 \\
\hline 0904 & $1.0000 \mathrm{~L}$ & $10.0000 \mathrm{NH}$ & 20.000011 & .0000 & 700,0000 & & & & 6011 & 100.0000 \\
\hline 200905 & 1.00002 & $10.0000 \mathrm{~N}$ & $20,0000 \mathrm{~N}$ & 0.0000 & 200.0000 & 1.0000 & 1000 & $5.0000 \mathrm{H}$ & $20.0000 \mathrm{~N}$ & 50.0000 \\
\hline 200906 & 1.00001 & $10.0000 \mathrm{~N}$ & $20.0000 \mathrm{~N}$ & 0.0000 & & 0000 & 20.0000 & $5.0000 \mathrm{~N}$ & $1000 \mathrm{H}$ & 70.0000 \\
\hline 0907 & & & & & & & & & OON & 70.0000 \\
\hline 200908 & & $\mathrm{OOH}$ & & & & & & & OON & 000 \\
\hline 200909 & 0001 & $10.0000 \mathrm{H}$ & $20.0000 \mathrm{H}$ & 10.0000 & 100 & 000 & .0000 & 0.0000 & & 000 \\
\hline 200910 & $1,0000 L$ & 10.000011 & $20.0000 \mathrm{~N}$ & & & & & & & 15.0000 \\
\hline 200911 & & & & & & & & & & .0000 \\
\hline 200912 & $1.0000 \mathrm{~L}$ & $10.0000 \mathrm{H}$ & OON & & & & & & OON & 70.0000 \\
\hline T & $1.0000 \mathrm{~L}$ & $\mathrm{OOH}$ & & & & & & & & \\
\hline & & & & & & & & & & \\
\hline $\begin{array}{l}200915 \\
200916\end{array}$ & & & & $\begin{array}{l}00 \\
00\end{array}$ & & & & & & \\
\hline 2009 & & & & & & & & & & 50.0000 \\
\hline 200918 & 1.00006 & 10.0 & OON & .0000 & & & & & & 50.0000 \\
\hline 0910 & 1.00001 & & & 0000 & & & & & & 50.0000 \\
\hline 00920 & 10L & & ON & $\infty 0$ & & & & & $\mathrm{OON}$ & 0000 \\
\hline 200921 & $10 \mathrm{~L}$ & 011 & & .0000 & 200 & & & & OON & 100 \\
\hline 0922 & & & & .0000 & 300 & & & & $\mathrm{OOH}$ & 15.0000 \\
\hline 200923 & 1.00001 & 10.0 & $20.0000 \mathrm{~N}$ & .0000 & 1000.0000 & & & DOH & 10011 & 150.0000 \\
\hline 200924 & 1.00002 & ON & $20.0000 \mathrm{~N}$ & .0000 & 00 & & & $\mathrm{OOH}$ & OON & 100.0000 \\
\hline 5 & OL & & & & & & & & & 70.0000 \\
\hline 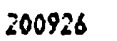 & & & & & & & & & OONI & 100.0000 \\
\hline 6927 & 1.00001 & 10. & & & 00 & & & & $\mathrm{OOH}$ & 20.0000 \\
\hline 0928 & & & & & & & & & & 70.0000 \\
\hline & & & & & & & & & & \\
\hline $\begin{array}{l}00930 \\
00931\end{array}$ & & ON & $\begin{array}{l}N \\
N\end{array}$ & & & & & ONN & & 100 \\
\hline 2009931 & & & & & & & & & & \\
\hline דדרמח & 10006 & & & 100 & & 00 & & ON & & 000 \\
\hline & & & & & & & & & & \\
\hline & & & & & & & & & & 000 \\
\hline 0 07s & & & & & & & & & & \\
\hline ירםחט & & & N & & & 0 & & & & \\
\hline & & & & & & & & & & 50 \\
\hline 00939 & 00001 & oll & & & & & & & & \\
\hline & & & & & & & & & & \\
\hline & & & & & & & & & & \\
\hline 200942 & 1.00002 & ONH & & 1000 & & 000 & & 10011 & & \\
\hline 200943 & & & & & & & & & & \\
\hline & & & & & & & & & & \\
\hline & & & & & & & & & & 50 \\
\hline & & & & & & & & & & \\
\hline & 1.00001 & $10.0000 \mathrm{~N}$ & $20.0000 \mathrm{~N}$ & 0.0000 & 500.0000 & 20.0000 & 30.0000 & 5.000011 & $20.0000 \mathrm{~L}$ & 50.0000 \\
\hline
\end{tabular}


Table 1.--Analytical data for rock chip sampling--Continued

FRINTOUT FOR SPEC/AA

\begin{tabular}{|c|c|c|c|c|c|c|c|c|c|c|}
\hline SARFLLE & $S-F P$ & $S-S B$ & $S-S C$ & $5-S N$ & $5-5 R$ & $s-v$ & $S-W$ & $S-Y$ & $S-2 N$ & $s-2 R$ \\
\hline $\begin{array}{l}200898 \\
200889\end{array}$ & $\begin{array}{l}50.0000 \\
10.0000 \mathrm{~L}\end{array}$ & $\begin{array}{l}100.0000 \mathrm{~N} \\
100.0000 \mathrm{~N}\end{array}$ & $\begin{array}{l}15.0000 \\
15.0000\end{array}$ & $\begin{array}{l}10.0000 \mathrm{~N} \\
10.000 \mathrm{~N}\end{array}$ & $\begin{array}{l}200,0000 \\
200.0000\end{array}$ & $\begin{array}{l}200,0000 \\
200,0000\end{array}$ & $\begin{array}{l}50.000011 \\
70.0000\end{array}$ & $\begin{array}{l}20.0000 \\
20.0000\end{array}$ & $\begin{array}{l}200.0000 \mathrm{~N} \\
200.000 \mathrm{CN}\end{array}$ & $\begin{array}{l}100.0000 \\
150.0000\end{array}$ \\
\hline 200900 & $10.0000 \mathrm{~L}$ & $100.0000 \mathrm{~N}$ & 15.0000 & $10.0000 \mathrm{~N}$ & 200.0000 & 150,0000 & 1000.0000 & 15.0000 & $200.0000 \mathrm{~N}$ & 100.0000 \\
\hline $\begin{array}{l}200901 \\
20090 ?\end{array}$ & $\begin{array}{l}10.00001 \\
10.00001\end{array}$ & $\begin{array}{l}100.000014 \\
100.000011\end{array}$ & $\begin{array}{l}5.0000 \\
5.0000\end{array}$ & $\begin{array}{l}10.0000 \mathrm{~N} \\
10.0000 \mathrm{~N}\end{array}$ & $\begin{array}{l}200.0000 \\
300.0000\end{array}$ & $\begin{array}{l}70.0000 \\
20.0000\end{array}$ & $\begin{array}{l}50.0000 \mathrm{~N} \\
50.0000 \mathrm{~N}\end{array}$ & $\begin{array}{l}15.0000 \\
50.0000\end{array}$ & $\begin{array}{l}200.0000 \mathrm{H} \\
200.0000 \mathrm{H}\end{array}$ & $\begin{array}{l}200.0000 \\
500.0000\end{array}$ \\
\hline 200903 & 10.00000 & $100.0000 \mathrm{~N}$ & 15.0000 & $10.0000 \mathrm{~N}$ & 500,0000 & 150.0000 & $50,0000 \mathrm{~N}$ & 15.0000 & $200.0000 \mathrm{~N}$ & 150.0000 \\
\hline 200904 & 30.0000 & 100.000011 & 15.0000 & $10.0000 \mathrm{H}$ & 200.0000 & 100.0000 & $50.0000 \mathrm{~N}$ & 10.0000 & $200.0000 \mathrm{~N}$ & 150.0000 \\
\hline 200905 & $10.0000 \mathrm{~L}$ & $100.0000 \mathrm{~N}$ & 15.0000 & $10.0000 \mathrm{H}$ & 500,0000 & 100.0000 & $50.0000 \mathrm{~N}$ & & OON & 000 \\
\hline 200906 & $10.0060 \mathrm{~L}$ & $100.0000 \mathrm{~N}$ & 15.0000 & $10.0000 \mathrm{~N}$ & 200.0000 & 150.0000 & 50,000011 & 20.0000 & $200.0000 \mathrm{H}$ & 100.0000 \\
\hline $\begin{array}{l}200507 \\
200508\end{array}$ & $\begin{array}{l}10.0000 \mathrm{~N} \\
10.0000 \mathrm{H}\end{array}$ & $\begin{array}{l}100.0000 \mathrm{~N} \\
100.0000 \mathrm{~N}\end{array}$ & $\begin{array}{l}30.0000 \\
15.0000\end{array}$ & $\begin{array}{l}10.0000 \mathrm{~N} \\
10.0000 \mathrm{~N}\end{array}$ & & & & & $\begin{array}{l}200.000011 \\
200.000011\end{array}$ & \\
\hline 200909 & 10.0000 & $100.0000 \mathrm{H}$ & 15.0000 & 30.000011 & 500.0000 & 100.0000 & $50.0000 \mathrm{~N}$ & 20.0000 & $200.0000 \mathrm{~N}$ & 150.000 \\
\hline 200910 & OON & $100.0000 \mathrm{~N}$ & 5.0000 & $10.0000 \mathrm{~N}$ & 00 & 000 & ON & 10 & 200 & 150.000 \\
\hline 200911 & Voll & $100.0000 \mathrm{~N}$ & 15.0000 & OON & & 100 & & & OON & 200.0000 \\
\hline 200913 & 1001 & Onon & 50,0000 & OON & 100 & 200. & OON & 30 & 200. & 100.0000 \\
\hline 200913 & $.0003 \mathrm{~N}$ & $100.0000 \mathrm{~N}$ & 20.0000 & $10.0000 \mathrm{~N}$ & 00 & 150. & $\mathrm{OHH}$ & 30 & OONH & 150.0000 \\
\hline 200914 & $.0000 \mathrm{~N}$ & & & OON & & & & & JON & 200.0000 \\
\hline 200915 & OOH & 100 & 30.0000 & OON & & 200 & DON & 100 & OOH & 100.0000 \\
\hline 200918 & soll & 100. & 20.0000 & $\mathrm{OOH}$ & & 150. & OON & & OOH & \\
\hline 200917 & DCAs & 100 & 15.0000 & Oons & & 000 & 00 & 20. & 0011 & 150 \\
\hline 200918 & $.0000 \mathrm{~N}$ & 100.0 & 15.0000 & OON & 500.0000 & 150.0000 & OOON & 20. & OON & \\
\hline 200919 & OON & 100N & 15.0000 & OON & 00 & 200 & JON & 000 & 10OH & 150.0000 \\
\hline 200 & 011 & & & & & & & & $O N$ & \\
\hline 260921 & OON & 100.0 & 15.0000 & OON & & 200 & OON & & OOH & 150 \\
\hline 200922 & 0014 & & & & & & & & OON & 1000 \\
\hline 200923 & SON & 100 & 80 & 10. & & 108 & $O N$ & & OON & 100 \\
\hline 200924 & OON & 100. & 15.0 & $\mathrm{OON}$ & & 00 & ON & & OOH & 150.0000 \\
\hline 200925 & oon & & & & & & & & DON & 000 \\
\hline 200928 & & OOH & 15.0000 & OON & 50 & 100 & $O N$ & & OON & 150,000 \\
\hline 200927 & OON & OON & & OON & & & & & DOH & 150.000 \\
\hline & & & & & & & & & $O N$ & 150. \\
\hline 200929 & Soll & DOH & 5 & ON & & & & & ON & 900 \\
\hline 200930 & & 100.0 & & & & & & & DON & 000 \\
\hline & & & & & & & & & & 000 \\
\hline & & & & & & & & & & \\
\hline 200933 & DOON & $100.0000 \mathrm{H}$ & 50.0000 & 10.0 & & & OON & & OON & 100.0000 \\
\hline $\begin{array}{l}200934 \\
200935\end{array}$ & $\begin{array}{l}\text { Dor1 } \\
\text { OON1 }\end{array}$ & $\begin{array}{l}\text { OONH } \\
\text { Oonit }\end{array}$ & $\begin{array}{l}15.0000 \\
50.0000\end{array}$ & 001 & $\begin{array}{l}00 \\
00\end{array}$ & & & & $\begin{array}{l}\text { OON } \\
\text { OON }\end{array}$ & $\begin{array}{l}100.0000 \\
100.0000\end{array}$ \\
\hline 200536 & OON & ONW & 50.0 & $\mathrm{ONN}$ & & & & & OON & 100.0000 \\
\hline 200937 & $10.0000 \mathrm{H}$ & $100.0000 \mathrm{~N}$ & 20.0000 & 10.0 & 300.0 & & $100 N$ & & $200.0000 \mathrm{H}$ & 200.000 \\
\hline 2009 & & OOH & & OON & & & & & & \\
\hline 200939 & $0000 \mathrm{Ht}$ & $100.0000 \mathrm{H}$ & 20.0000 & $10.0000 \mathrm{~N}$ & 300.0000 & 150.0000 & OON & 20.0000 & $200.0000 \mathrm{~N}$ & 200.000 \\
\hline 200940 & 00018 & $100.0000 \mathrm{~N}$ & 20.0000 & $10.0000 \mathrm{~N}$ & & 150.0000 & OON & 20.0000 & $200.0000 \mathrm{H}$ & 200.000 \\
\hline & & & & & & & & & & 150.000 \\
\hline 200942 & $10.0000 \mathrm{~N}$ & $100.0000 \mathrm{~N}$ & 20.0000 & $10.0000 \mathrm{~N}$ & 300.0 & & 1011 & & & 150.000 \\
\hline 200943 & $10.0000 \mathrm{~N}$ & $100.0000 \mathrm{~N}$ & 30.0000 & $10.0000 \mathrm{~N}$ & 500,0000 & 200,0000 & OOON & 20.0000 & $200.0000 \mathrm{~N}$ & 150.000 \\
\hline 200944 & $10.0000 \mathrm{~N}$ & $100,0000 \mathrm{~N}$ & 20.0000 & 10.000011 & & & & & $200.0000 \mathrm{H}$ & 200.000 \\
\hline 200945 & $10.0000 \mathrm{~N}$ & 100,000011 & & & 300.0000 & & $000 \mathrm{~N}$ & $10,0000 \mathrm{~L}$ & $200.0000 \mathrm{~N}$ & 100.000 \\
\hline 200946 & $10.0000 \mathrm{~N}$ & 100.000011 & 15.0000 & $10.0000 \mathrm{H}$ & 500,0000 & 150.0000 & $50.0000 \mathrm{H}$ & 20.0000 & $200.0000 \mathrm{~N}$ & 150.000 \\
\hline 200547 & $10.0000 \mathrm{~N}$ & $100.0000 \mathrm{~N}$ & 20.0000 & $10.0000 \mathrm{~N}$ & 1000,0000 & 100.0000 & $50.0000 \mathrm{~N}$ & 30.0000 & $200.0000 \mathrm{H}$ & 150.000 \\
\hline
\end{tabular}


Table 1.--Analytical data for rock chip sampling--Continued

PRINTOUT FOR SPEC/AA

\begin{tabular}{|c|c|c|}
\hline SAMPLE & $A A-A U-P$ & $A Z-A G$ \\
\hline $\begin{array}{l}200898 \\
2008999\end{array}$ & $\begin{array}{l}0.0500 \mathrm{~N} \\
0.0600\end{array}$ & $\begin{array}{l}0.1000 \mathrm{~N} \\
0.1000 \mathrm{~N}\end{array}$ \\
\hline 200900 & 0.0700 & 0.1700 \\
\hline $\begin{array}{l}200901 \\
200902\end{array}$ & $\begin{array}{l}0.1800 \\
0.0500 \mathrm{~N}\end{array}$ & $\begin{array}{l}0.1000 \mathrm{~N} \\
0.1000 \mathrm{~N}\end{array}$ \\
\hline $\begin{array}{l}200903 \\
200904 \\
260905\end{array}$ & $\begin{array}{l}0.0500 \mathrm{~N} \\
0.0500 \mathrm{H} \\
0.0500 \mathrm{~N}\end{array}$ & $\begin{array}{l}0.1000 \mathrm{~N} \\
0.1000 \mathrm{~N} \\
0.1000 \mathrm{~N}\end{array}$ \\
\hline 200906 & $0.0500 \mathrm{H}$ & $0.1000 \mathrm{~N}$ \\
\hline $\begin{array}{l}200507 \\
206908\end{array}$ & $\begin{array}{l}0.0500 \mathrm{H} \\
0.0500 \mathrm{~K}\end{array}$ & $\begin{array}{l}0,1000 \mathrm{~N} \\
0,1000 \mathrm{H}\end{array}$ \\
\hline 200909 & $0.0500 \mathrm{~N}$ & $0,1000 \mathrm{~N}$ \\
\hline $\begin{array}{l}200910 \\
200911\end{array}$ & $\begin{array}{l}0.050011 \\
0.05001\end{array}$ & $\begin{array}{l}0.1000 \mathrm{~N} \\
0.1000 \mathrm{~N}\end{array}$ \\
\hline 200912 & $0.0500 \mathrm{~N}$ & $0.1000 \mathrm{~N}$ \\
\hline $\begin{array}{l}200913 \\
200911\end{array}$ & $\begin{array}{l}0.0500 \mathrm{~N} \\
0.0500\end{array}$ & $\begin{array}{l}0.10001 \\
0.1000\end{array}$ \\
\hline 200915 & $0.0500 \mathrm{~N}$ & $0.1000 \mathrm{~N}$ \\
\hline 200916 & $0.0500 \mathrm{~N}$ & 0.1200 \\
\hline 200917 & $0.0500 \mathrm{~L}$ & $0.1000 \mathrm{~L}$ \\
\hline 200918 & $0.0500 \mathrm{~N}$ & $0.1000 \mathrm{~N}$ \\
\hline 200919 & $0.0500 \mathrm{~N}$ & $0.1000 \mathrm{~N}$ \\
\hline 200920 & $0.0500 \mathrm{~N}$ & $0.1000 \mathrm{~N}$ \\
\hline 200921 & $0.0500 \mathrm{~N}$ & $0.1000 \mathrm{~N}$ \\
\hline 200922 & $0,0500 \mathrm{~L}$ & 0.2800 \\
\hline 200923 & $0.0500 \mathrm{~N}$ & $0.1000 \mathrm{~N}$ \\
\hline 200924 & $0.0500 \mathrm{~N}$ & $0.1000 \mathrm{~N}$ \\
\hline 200925 & $0.0500 \mathrm{~N}$ & $0.1000 \mathrm{~N}$ \\
\hline 200926 & $0.0500 \mathrm{H}$ & $0.1000 \mathrm{~L}$ \\
\hline 200527 & $0.0500 \mathrm{~N}$ & $0.1000 \mathrm{~N}$ \\
\hline $\begin{array}{l}200928 \\
200929\end{array}$ & $\begin{array}{l}0.0500 \mathrm{~N} \\
0.0500 \mathrm{~N}\end{array}$ & $\begin{array}{l}0.1000 \mathrm{~N} \\
0,1000 \mathrm{~N}\end{array}$ \\
\hline 200930 & 0.05001 & 0.4000 \\
\hline $\begin{array}{l}200931 \\
200932\end{array}$ & $\begin{array}{l}0.0500 \mathrm{~N} \\
0.0500 \mathrm{~N}\end{array}$ & $\begin{array}{l}0.1000 \mathrm{~N} \\
0.1000 \mathrm{~N}\end{array}$ \\
\hline 200933 & $0.0500 \mathrm{~N}$ & $0.1000 \mathrm{~N}$ \\
\hline 200934 & $0.0500 \mathrm{~N}$ & $0.1000 \mathrm{~K}$ \\
\hline 200935 & $0.0500 \mathrm{~N}$ & $0.1000 \mathrm{~L}$ \\
\hline 200936 & $0.0500 \mathrm{~L}$ & $0.1000 \mathrm{~N}$ \\
\hline 200937 & $0.0500 \mathrm{~N}$ & $0.1000 \mathrm{~N}$ \\
\hline 200938 & $0.0500 \mathrm{~N}$ & $0.1000 \mathrm{~N}$ \\
\hline 200939 & $0.0500 \mathrm{~N}$ & $0.1000 \mathrm{n}$ \\
\hline $\begin{array}{l}200940 \\
200941\end{array}$ & $\begin{array}{l}0.0500 \mathrm{~N} \\
0.0500 \mathrm{~L}\end{array}$ & $\begin{array}{l}0.1000 \mathrm{~N} \\
0.1000 \mathrm{~L}\end{array}$ \\
\hline 200942 & $0.0500 \mathrm{~N}$ & $0.1000 \mathrm{~N}$ \\
\hline 200943 & $0.0500 \mathrm{~N}$ & $0,1000 \mathrm{H}$ \\
\hline 200944 & $0.0500 \mathrm{~N}$ & 0.6000 \\
\hline 200945 & $0.0500 \mathrm{~N}$ & 0.1000 \\
\hline 200946 & $0,0500 \mathrm{~N}$ & $0.1000 \mathrm{~N}$ \\
\hline 200947 & $0.0500 N$ & $0.1000 \mathrm{~L}$ \\
\hline
\end{tabular}


Table 1.--Analytical data for rock chip sampling--Continued

PRINTOUT FOR SPEC/AA

\begin{tabular}{|c|c|c|c|c|c|c|c|c|c|c|}
\hline SAMFLE & S-FE & $5-K 0$ & $S-C A$ & S-II & S-5int & S-AG & S-AS & S-AU & $5-8$ & $5-8 A$ \\
\hline $\begin{array}{l}200948 \\
200949\end{array}$ & $\begin{array}{l}5.0000 \\
5.0000\end{array}$ & $\begin{array}{l}5.0000 \\
5.0000\end{array}$ & $\begin{array}{l}1.0000 \\
0.7000\end{array}$ & $\begin{array}{l}0.2000 \\
0.2000\end{array}$ & $\begin{array}{l}500.0000 \\
500.0000\end{array}$ & $\begin{array}{l}0.5000 \mathrm{~N} \\
0.5000 \mathrm{~N}\end{array}$ & $\begin{array}{l}200.0000 \mathrm{~N} \\
200.0000 \mathrm{~N}\end{array}$ & $\begin{array}{l}10.0000 \mathrm{~N} \\
10.000011\end{array}$ & $\begin{array}{l}10.0000 \\
30.0000\end{array}$ & $\begin{array}{l}300.0000 \\
500.0000\end{array}$ \\
\hline 200950 & 5.0000 & 5.0000 & 0.7000 & 0.2000 & 700.0000 & $0.5000 \mathrm{~N}$ & $200.0000 \mathrm{~N}$ & $10.0000 \mathrm{~N}$ & 20.0000 & 500.0000 \\
\hline $\begin{array}{l}200951 \\
200952\end{array}$ & $\begin{array}{l}5.0000 \\
5.0000\end{array}$ & $\begin{array}{l}5.0000 \\
5.0600\end{array}$ & $\begin{array}{l}2.0000 \\
2.0000\end{array}$ & $\begin{array}{l}0.2000 \\
0.2000\end{array}$ & $\begin{array}{r}700.0000 \\
1000.0000\end{array}$ & $\begin{array}{l}0.5000 \mathrm{~N} \\
0.5000 \mathrm{~N}\end{array}$ & $\begin{array}{l}200.0000 \mathrm{H} \\
200.0000 \mathrm{~N}\end{array}$ & $\begin{array}{l}10,000011 \\
10.000011\end{array}$ & $\begin{array}{l}20.0000 \\
20.0000\end{array}$ & $\begin{array}{l}300.0000 \\
200.0000\end{array}$ \\
\hline 200953 & 5.0000 & 7.0000 & 3,0000 & 0.3000 & 1000.0000 & $0.5000 \mathrm{H}$ & $200.0600 \mathrm{~N}$ & $10.0000 \mathrm{~N}$ & 10.0000 & 70.0000 \\
\hline $\begin{array}{l}200954 \\
200955\end{array}$ & $\begin{array}{l}3.0000 \\
3.0000\end{array}$ & $\begin{array}{l}3.0000 \\
5.0000\end{array}$ & $\begin{array}{r}1.5000 \\
10.0000\end{array}$ & $\begin{array}{l}0.2000 \\
0.2000\end{array}$ & $\begin{array}{r}500.0000 \\
2000.0000\end{array}$ & $\begin{array}{l}0.5000 \mathrm{~N} \\
0.5000 \mathrm{~N}\end{array}$ & $\begin{array}{l}200.0000 \mathrm{~N} \\
200.0000 \mathrm{H}\end{array}$ & $\begin{array}{l}10.000011 \\
10.0000 \mathrm{~N}\end{array}$ & $\begin{array}{l}10.0000 \\
10.0000 \mathrm{~L}\end{array}$ & $\begin{array}{r}100.0000 \\
1000.0000\end{array}$ \\
\hline 200956 & 3.0000 & 5,0000 & 5.0000 & 0.2000 & 1000,0000 & $0.5000 \mathrm{~N}$ & $200.0000 \mathrm{~N}$ & $10.0000 \mathrm{~N}$ & 10.0000 & 700.0000 \\
\hline 200957 & 7.0000 & 7.0000 & 5.0000 & 0.5000 & 1000.0000 & $0.5000 \mathrm{~N}$ & $200.0000 \mathrm{H}$ & $10.0000 \mathrm{~N}$ & 20.0000 & 200.0000 \\
\hline 200958 & 5,0000 & 5.0000 & 5,0000 & 0.2000 & 1000,0000 & $0.5000 \mathrm{~N}$ & $200,0000 \mathrm{~N}$ & $10.0000 \mathrm{~N}$ & 20,0000 & 100.0000 \\
\hline 200959 & 5.0000 & 5.0000 & 7.0000 & 0.2000 & 1000.0000 & $0.5000 \mathrm{H}$ & $200.0000 \mathrm{~N}$ & $10.0000 \mathrm{~N}$ & 15.0000 & 1500,0000 \\
\hline 200960 & 5.0000 & 5.6000 & 1.0000 & 0.2000 & 500.0000 & $0.5000 \mathrm{~N}$ & $200.0000 \mathrm{H}$ & $10.0000 \mathrm{~N}$ & 20.0000 & 700.0000 \\
\hline 2049961 & 5.0000 & 2.0000 & 1.0000 & 0.2000 & 500.0000 & $0.5000 \mathrm{~N}$ & $200.0000 \mathrm{~N}$ & $10.0000 \mathrm{~N}$ & 20,0000 & 300.0000 \\
\hline 200962 & 5.0000 & 5.0000 & 1.0000 & 0.2000 & 500.0000 & $0.5000 \mathrm{~N}$ & $200,0000 \mathrm{~N}$ & 10.000011 & 15.0000 & 500.0000 \\
\hline $\begin{array}{l}200963 \\
200964\end{array}$ & $\begin{array}{l}5.0000 \\
5.0000\end{array}$ & $\begin{array}{l}2.0000 \\
5.0000\end{array}$ & $\begin{array}{l}1.0000 \\
2.0000\end{array}$ & $\begin{array}{l}0.2000 \\
0.2000\end{array}$ & $\begin{array}{l}500.0000 \\
700.0000\end{array}$ & $\begin{array}{l}0.5000 \mathrm{~N} \\
0.5000 \mathrm{~N}\end{array}$ & $\begin{array}{l}200.0000 \mathrm{~N} \\
200.000011\end{array}$ & $\begin{array}{l}10.0000 \mathrm{~N} \\
10.0000 \mathrm{~N}\end{array}$ & $\begin{array}{l}10.0000 \\
20.0000\end{array}$ & $\begin{array}{l}500.0000 \\
500.0000\end{array}$ \\
\hline 200965 & 5.0000 & 5.0000 & 1,0000 & 0.2000 & 500.0000 & $0.5000 \mathrm{~N}$ & $200.0000 \mathrm{~N}$ & $10.0000 \mathrm{~N}$ & 20.0000 & 500,0000 \\
\hline 200966 & 5.0000 & 5.0000 & 1,0000 & 0.3000 & 700.0000 & $0.5000 \mathrm{~N}$ & $200.0000 \mathrm{~N}$ & $10.0000 \mathrm{H}$ & 20.0000 & 500.0000 \\
\hline 200967 & 5.0000 & 5.0000 & 1.0000 & 0.3000 & 500.0000 & $0.5000 \mathrm{H}$ & $200.0000 \mathrm{H}$ & $10.0000 \mathrm{~N}$ & 20.0000 & 300.0000 \\
\hline 200968 & 5.0000 & 5.0000 & 2.0000 & 0.2000 & 700.0000 & $0.5000 \mathrm{~N}$ & $200.0000 \mathrm{~N}$ & $10.0000 \mathrm{~N}$ & 10.0000 & 500,0000 \\
\hline 200969 & 5.0000 & 5.0000 & 0.3000 & 0.2000 & 500.0000 & $0.5000 \mathrm{~N}$ & 200.000011 & $10.0000 \mathrm{~N}$ & $10.0000 \mathrm{~L}$ & 200.0000 \\
\hline 200970 & 5.0000 & 5.0000 & 0.5000 & 0.2000 & 300,0000 & $0.5000 \mathrm{~N}$ & $200.0000 \mathrm{~N}$ & $10.0000 \mathrm{~N}$ & 10.0000 & 300.0000 \\
\hline 200971 & 5.0000 & 5.0000 & 5.0000 & 0.2000 & 1500.0000 & $0.5000 \mathrm{~N}$ & $200.0000 \mathrm{~N}$ & $10.0000 \mathrm{~N}$ & 10.0000 & 700.0000 \\
\hline 200972 & 5.0600 & 5.0000 & 0.5000 & 0.2000 & 500.0000 & $0.5000 \mathrm{~N}$ & $200.0000 \mathrm{~N}$ & $10,0000 \mathrm{~N}$ & 10.0000 & 500,0000 \\
\hline 200973 & 5.0000 & 5.0000 & 10.0000 & 0.2000 & 2000.0000 & $0.5000 \mathrm{~N}$ & $200.0000 \mathrm{~N}$ & $10.0000 \mathrm{H}$ & $10.0000 \mathrm{~L}$ & 700.0000 \\
\hline 200974 & 5,0000 & 5.0000 & 1.0000 & 0.2600 & 500.0000 & $0.5000 \mathrm{~N}$ & $200.0000 \mathrm{~N}$ & $10.0000 \mathrm{~N}$ & 10.0000 & 500.0000 \\
\hline $\begin{array}{l}200975 \\
200976\end{array}$ & $\begin{array}{l}5.0000 \\
5.0600\end{array}$ & $\begin{array}{l}5.0000 \\
5.0000\end{array}$ & $\begin{array}{l}1.0000 \\
0.7000\end{array}$ & $\begin{array}{l}0.2000 \\
0.2000\end{array}$ & $\begin{array}{l}700.0000 \\
500.0000\end{array}$ & $\begin{array}{l}0.5000 \mathrm{H} \\
0.5000 \mathrm{~N}\end{array}$ & $\begin{array}{l}200.0000 \mathrm{~N} \\
200.0000 \mathrm{~N}\end{array}$ & $\begin{array}{l}10.0000 \mathrm{~N} \\
10.0000 \mathrm{~N}\end{array}$ & $\begin{array}{l}30.0000 \\
30.0000\end{array}$ & $\begin{array}{l}500.0000 \\
700.0000\end{array}$ \\
\hline 200977 & 5.0000 & 5.0000 & 1.0000 & 0.2000 & 500.0000 & $0.5000 \mathrm{H}$ & 200.000011 & $10.0000 \mathrm{~N}$ & 10,0060 & 300.0000 \\
\hline 200978 & 5.0000 & 5.0000 & 1.5000 & 0.2000 & 700.0000 & $0.5000 \mathrm{~N}$ & $200.0000 \mathrm{~N}$ & $10.0000 \mathrm{~N}$ & 20.0000 & 200.0000 \\
\hline 200979 & 5.0000 & 7.0000 & 2.0000 & 0.2000 & 700.0000 & $0.5000 \mathrm{H}$ & $200.0000 \mathrm{~N}$ & $10.0000 \mathrm{~N}$ & 20,0000 & 100.0000 \\
\hline
\end{tabular}


Table 1.--Analytical data for rock chip sampling--Continued

PRINTOUT FOR SPEC/AA

\begin{tabular}{|c|c|c|c|c|c|c|c|c|c|c|}
\hline SAYPLLE & S-BE & $S-B I$ & $S-C D$ & $5-C 0$ & $5-C R$ & s-cu & $S-L A$ & $5-150$ & S-NB & $-N I$ \\
\hline 1948 & $\begin{array}{l}1.0000 \mathrm{~L} \\
1.0000 \mathrm{~L}\end{array}$ & $\begin{array}{l}10.0000 \mathrm{~N} \\
10.0000 \mathrm{~N}\end{array}$ & $\begin{array}{l}20.0000 \mathrm{~N} \\
20.0000 \mathrm{H}\end{array}$ & $\begin{array}{l}.0000 \\
.0000\end{array}$ & $\begin{array}{l}200.0000 \\
150.0000\end{array}$ & & & & & \\
\hline $\begin{array}{l}200950 \\
200951 \\
200952\end{array}$ & $\begin{array}{l}1.0000 L \\
1.0000 L \\
1.0000 L\end{array}$ & $\begin{array}{l}10.000011 \\
10.000011 \\
10.000011\end{array}$ & $\begin{array}{l}20.0000 \mathrm{~N} \\
20.0000 \mathrm{~N} \\
20.0000 \mathrm{~N}\end{array}$ & 0.0000 & $\begin{array}{l}100.0000 \\
150.0000 \\
100.0000\end{array}$ & $\begin{array}{l}0.0000 \\
0.0000 \\
10.0000\end{array}$ & & $\begin{array}{l}5.0000 \mathrm{~N} \\
5.0000 \mathrm{~N} \\
5.0000 \mathrm{H}\end{array}$ & $\begin{array}{l}20.0000 \mathrm{~N} \\
20.0000 \mathrm{~N} \\
20.0000 \mathrm{~N}\end{array}$ & $\begin{array}{l}20.0000 \\
50.0000 \\
50.0000\end{array}$ \\
\hline 200953 & a & $.0000 \mathrm{NH}$ & $20.0000 \mathrm{~N}$ & .0000 & 150.0000 & 0.0000 & .0000 & OOON & $0.0000 \mathrm{~N}$ & 70.0000 \\
\hline 200954 & & & 20.0 & 0.0000 & 100.0000 & .0000 & & $\mathrm{OCH}$ & OOH & .0000 \\
\hline 200955 & & & & & 00 & & & $100 \mathrm{~N}$ & $100 \mathrm{~N}$ & .0000 \\
\hline 200956 & 1.00002 & $.0000 \mathrm{~K}$ & & & 150.0000 & & & $000 \mathrm{H}$ & OON & 0.0000 \\
\hline 200957 & & & & & & & & & & \\
\hline & & & & & & & & & & \\
\hline 200959 & 1.0 & OHI & 20.0 & 100 & 200.0000 & 100 & 00 & DOOH & $100 \mathrm{~N}$ & 50.0000 \\
\hline 200960 & & & & & & & & & & 000 \\
\hline 2009 & & & & & & & & & DON & 1000 \\
\hline $\begin{array}{l}200962 \\
200963\end{array}$ & & & & & & & & & & \\
\hline 200964 & 1.0 & $100 \mathrm{~N}$ & ON & 00 & 00 & 100 & & & OOON & 50.0000 \\
\hline 200965 & 1,00002 & 000011 & $20.0000 \mathrm{H}$ & 20.0000 & 000 & .0000 & & 1002 & OOOH & 50.0000 \\
\hline 200966 & & $1000 \mathrm{~N}$ & & & & & & OOL & OOON & 0000 \\
\hline & & & & & & & & & OOH & \\
\hline 200968 & & ON & 20. & $\infty 0$ & 700 & 100 & & OON & OOH & 1000 \\
\hline 200969 & & & 20. & 20. & 200 & 00 & OOL & OOH & OOON & 70.0000 \\
\hline 2009 & & 000N" & $O N$ & & & & & & $000 \mathrm{~N}$ & 50.0000 \\
\hline 200971 & 3.0000 & 10.00064 & 20.000011 & 15.0000 & 150. & .0000 & 0001 & 0011 & $1000 \mathrm{~N}$ & 30.0000 \\
\hline 200972 & & OOON & $20.0000 \mathrm{~N}$ & & 200.0 & .0000 & & OOH & & \\
\hline & & & & & & & & & & \\
\hline 200974 & 1.00002 & $0000 \mathrm{~N}$ & ON & 20.0000 & 300.0000 & 20.0000 & 20.0000 & $5.0000 \mathrm{H}$ & $20.0000 \mathrm{~N}$ & 50.0000 \\
\hline 200975 & $1.0000 \mathrm{~L}$ & 10.000011 & & 30.0000 & & & $20.0000 \mathrm{~L}$ & $5.0000 \mathrm{~N}$ & DOON & 20.0000 \\
\hline & & & & & & & & & & 70.0000 \\
\hline & $.0000 \mathrm{~N}$ & & $20.0000 \mathrm{~N}$ & 20.0000 & 200.0000 & & & & & 70.0000 \\
\hline & $\begin{array}{l}1.0000 \mathrm{~N} \\
1.0000 \mathrm{~N}\end{array}$ & OOOH & & & $\begin{array}{l}200 \\
200\end{array}$ & & & $\mathrm{OOH}$ & & $\begin{array}{l}50.0000 \\
50.0000\end{array}$ \\
\hline & & & & & & 00 & & & $20.0000 \mathrm{~N}$ & \\
\hline
\end{tabular}


Table 1.--Analytical data for rock chip sampling--Continued

PRINTOUT FOR SPEC/AA

\begin{tabular}{|c|c|c|c|c|c|c|c|c|c|c|}
\hline SAMPLE & $S-F B$ & S-SB & $5-s c$ & S-SN & S-SR & $s-v$ & $s-4$ & S-Y & $s-2 N$ & $s-2 R$ \\
\hline 0948 & DOOLL & $\begin{array}{l}100.0000 \mathrm{~N} \\
100.0000 \mathrm{~N}\end{array}$ & 100 & $\begin{array}{l}.0000 \mathrm{H} \\
1.0000 \mathrm{H}\end{array}$ & $\begin{array}{l}300.0000 \\
200.0000\end{array}$ & $\begin{array}{l}100.0000 \\
100.0000\end{array}$ & DOON & $\begin{array}{l}20.0000 \\
20.0000\end{array}$ & $\begin{array}{l}200.0000 \mathrm{H} \\
200.0000 \mathrm{~N}\end{array}$ & \\
\hline 200950 & $10.0000 \mathrm{~L}$ & $100.0000 \mathrm{~N}$ & 0.0000 & 10.00004 & 500,0000 & 70.0000 & $50.0000 \mathrm{H}$ & 15.0000 & $200.0000 \mathrm{H}$ & 100,0000 \\
\hline $\begin{array}{l}200951 \\
200552\end{array}$ & $\begin{array}{l}.0000 \mathrm{~L} \\
.0000 \mathrm{~L}\end{array}$ & $\begin{array}{l}100.0 \\
100.0\end{array}$ & $\begin{array}{l}0.0000 \\
5.0000\end{array}$ & $\begin{array}{l}10.0000 \mathrm{H} \\
10.0000 \mathrm{H}\end{array}$ & $\begin{array}{l}300.0000 \\
200.0000\end{array}$ & $\begin{array}{l}100.0000 \\
100.0000\end{array}$ & $\begin{array}{l}50.0000 \mathrm{~N} \\
50.000011\end{array}$ & & $\begin{array}{l}0.0000 \mathrm{H} \\
0.0000 \mathrm{~N}\end{array}$ & \\
\hline 00953 & 10.00001 & $100.0000 \mathrm{~N}$ & .0000 & $.0000 N$ & 200.0000 & 200.0000 & $0.0000 \mathrm{~N}$ & .0000 & .000014 & 70.0000 \\
\hline $\begin{array}{l}0954 \\
10955\end{array}$ & & & & & & & & & & \\
\hline A & & & & & & & & & & 100.0000 \\
\hline & & & & & & & & & & \\
\hline & & & & & & & & & & 000 \\
\hline 0959 & & & & & & & & & & \\
\hline 60 & & & & & & & & & & \\
\hline & & & & & & & & & & \\
\hline & & & & & & & & & & \\
\hline & & & & & & & & & & \\
\hline 65 & & & & & & & $\mathrm{OHH}$ & 000 & OOH & 0000 \\
\hline & & & & & & & & & & \\
\hline & & & & & & & & & & \\
\hline 1000 & & & & & & & & 1000 & & \\
\hline & & & & & & & & 000 & & 000 \\
\hline & & & & & & & & & & \\
\hline 0971 & & ONH & & & & & & & & \\
\hline & & & & & & & & & & \\
\hline & & & & & & & & & & \\
\hline 200974 & & & & & & & & & & \\
\hline & & & & & & & & & & 1000 \\
\hline 200 & & & & & & & & & & 100.0000 \\
\hline & & & & & & & & & & \\
\hline 60979 & & & & & & & & & $200.0000 \mathrm{~N}$ & 300.006 \\
\hline
\end{tabular}


Table 1.--Analytical data for rock chip sampling--Continued

PRINTOUT FOR SFEC/AA

\begin{tabular}{|c|c|c|}
\hline SAYFLE & $A A-A U-P$ & $A Z-A G$ \\
\hline $\begin{array}{l}200948 \\
200949\end{array}$ & $\begin{array}{l}0.0500 \mathrm{~N} \\
0.0500 \mathrm{~L}\end{array}$ & $\begin{array}{l}0.10001 \\
0.1500\end{array}$ \\
\hline 200950 & $0.0500 \mathrm{~N}$ & 0.10001 \\
\hline $\begin{array}{l}200951 \\
200952\end{array}$ & $\begin{array}{l}0.0500 \mathrm{~N} \\
0.0500 \mathrm{~N}\end{array}$ & $\begin{array}{l}0.1100 \\
0.1000 L\end{array}$ \\
\hline $\begin{array}{l}200953 \\
200954\end{array}$ & $\begin{array}{l}0.0500 \mathrm{~N} \\
0.0500 \mathrm{~N}\end{array}$ & $\begin{array}{l}0.1000 L \\
0.1000 L\end{array}$ \\
\hline 200955 & $0.0500 \mathrm{~N}$ & 0.1500 \\
\hline $\begin{array}{l}200956 \\
200957 \\
200958\end{array}$ & $\begin{array}{l}0.0500 \mathrm{H} \\
0.0500 \mathrm{~L} \\
0.0500 \mathrm{~N}\end{array}$ & $\begin{array}{l}0.1000 L \\
0.1000 \\
0.1000 L\end{array}$ \\
\hline 200959 & $0.0500 \mathrm{~L}$ & 0.1700 \\
\hline 200960 & $0.0500 \mathrm{~N}$ & $0.1000 \mathrm{~L}$ \\
\hline 200961 & $0.0500 \mathrm{~N}$ & $0.1000 \mathrm{~L}$ \\
\hline 200962 & $0.0500 \mathrm{~N}$ & 0.1000 \\
\hline 200963 & $0.0500 \mathrm{~N}$ & $0.1000 \mathrm{~L}$ \\
\hline 200964 & $0.0500 \mathrm{~N}$ & 0,1000 \\
\hline 200965 & $0.0500 \mathrm{~N}$ & 0.1100 \\
\hline 200966 & $0,0500 \mathrm{~N}$ & $0.1000 \mathrm{~L}$ \\
\hline 200967 & $0.0500 \mathrm{~N}$ & 0.1500 \\
\hline 200968 & $0.0500 \mathrm{H}$ & $0,1000 \mathrm{~L}$ \\
\hline 200969 & $0.0500 \mathrm{~N}$ & $0,1000 \mathrm{~L}$ \\
\hline 200970 & 0.050011 & 0.1500 \\
\hline 200971 & $0.0500 \mathrm{~N}$ & 0.1000 \\
\hline $\begin{array}{l}200972 \\
200973\end{array}$ & $\begin{array}{l}0.0500 \mathrm{~N} \\
0.0500 \mathrm{~N}\end{array}$ & $\begin{array}{l}0.1000 L \\
0.1600\end{array}$ \\
\hline 200974 & $0.0500 \mathrm{~N}$ & 0,10002 \\
\hline 200975 & $0.0500 \mathrm{~N}$ & $0.1000 \mathrm{~L}$ \\
\hline 200976 & $0.0500 \mathrm{~N}$ & $0.1000 \mathrm{~L}$ \\
\hline 200977 & $0.050 \mathrm{CN}$ & $0.1000 \mathrm{~L}$ \\
\hline $\begin{array}{l}200978 \\
200979\end{array}$ & $\begin{array}{l}0.0500 \mathrm{~N} \\
0.0500 \mathrm{~N}\end{array}$ & $\begin{array}{l}0.1000 L \\
0.1100\end{array}$ \\
\hline
\end{tabular}

\begin{tabular}{|c|c|c|c|c|c|c|c|c|c|c|}
\hline SAMPLE & $S-F E$ & S-Ho & $S-C A$ & S-II & $5-5 M$ & $S-A G$ & S-AS & S-All & $S-B$ & $S-B A$ \\
\hline $\begin{array}{l}213170 \\
213171\end{array}$ & $\begin{array}{l}2.0000 \\
1.0000\end{array}$ & $\begin{array}{l}1.5000 \\
0.3000\end{array}$ & $\begin{array}{r}0.7000 \\
20.0000\end{array}$ & $\begin{array}{l}0.1500 \\
0.0300\end{array}$ & $\begin{array}{r}300.0000 \\
1000.0000\end{array}$ & $\begin{array}{l}0,5000 \mathrm{~N} \\
0.5000 \mathrm{H}\end{array}$ & $\begin{array}{l}200.0000 \mathrm{~N} \\
200.0000 \mathrm{~N}\end{array}$ & $\begin{array}{l}10,0000 \mathrm{H} \\
10,000 \mathrm{H}\end{array}$ & $\begin{array}{l}20,0000 \\
10,000011\end{array}$ & $\begin{array}{r}300,0000 \\
50.0000\end{array}$ \\
\hline 213172 & 1,5000 & 1,5000 & 0.5000 & 0,1500 & 300.0000 & $0.5000 \mathrm{~N}$ & $200.0000 \mathrm{~N}$ & $10.0000 \mathrm{H}$ & $10.0000 \mathrm{~L}$ & 150.0000 \\
\hline 213173 & 2.0000 & 3.0000 & 1,0000 & 0,1500 & 300,0000 & $0.5000 \mathrm{~N}$ & $200.0000 \mathrm{~N}$ & $10.0000 \mathrm{~N}$ & 10.0000 & 200.0000 \\
\hline 213174 & 1.5000 & 2,0000 & 2.0000 & 0.1500 & 700,0000 & 0.500011 & $200.0000 \mathrm{~N}$ & $10.0000 \mathrm{~N}$ & 15.0000 & 100.0000 \\
\hline 213175 & 2.0000 & 2.0000 & 0.5000 & 0.2000 & 700.0000 & $0.5000 \mathrm{H}$ & $200.0000 \mathrm{~N}$ & $10.0000 \mathrm{~N}$ & 20.0000 & 300.0000 \\
\hline $2131 / 6$ & 2.0000 & 5,0000 & 1.0000 & 0,2000 & 500,0000 & 0,500011 & $200.0000 \mathrm{~N}$ & $10.0000 \mathrm{~N}$ & $10.0000 \mathrm{~N}$ & 500.0000 \\
\hline 213177 & 2.0000 & 2.0000 & 0.5000 & 0,1500 & 500,0000 & $0.5000 \mathrm{H}$ & $200,0000 \mathrm{~N}$ & $10.0000 \mathrm{H}$ & $10,0000 \mathrm{~L}$ & 200.0000 \\
\hline 213178 & 5.0000 & 0.2080 & 0.5000 & 0,1000 & 100,0000 & $0.5000 \mathrm{~N}$ & $200,0000 \mathrm{~N}$ & $10.0000 \mathrm{H}$ & $10,0000 \mathrm{~L}$ & 100,0000 \\
\hline 213179 & 1.5000 & 200000 & 5.0000 & 0.1500 & 500.0000 & $0.5000 \mathrm{H}$ & $200.0000 \mathrm{~N}$ & $10,0000 \mathrm{~N}$ & $10.0000 \mathrm{~L}$ & 150,0000 \\
\hline 213180 & 1.5000 & 1.0000 & & & 300,0000 & $0.5000 \mathrm{H}$ & $200.000 \mathrm{CN}$ & $10.0000 \mathrm{~N}$ & 10.00001 & 150,0000 \\
\hline 213181 & 5,0000 & 0.1500 & 1.0000 & 0.1500 & 100.0000 & $0.5000 \mathrm{~N}$ & $200.0000 \mathrm{~N}$ & $10.0000 \mathrm{~N}$ & $10.0000 \mathrm{~L}$ & 150.0000 \\
\hline 213182 & 3.0000 & 3.0000 & 0.2000 & 0.3000 & 200,0000 & $0.5000 \mathrm{H}$ & $200,0000 \mathrm{H}$ & $10.0000 \mathrm{~N}$ & 50.0000 & 500.0000 \\
\hline 213183 & 5,0000 & 2,0000 & 2,0000 & 1.0000 & 700,0000 & $0.5000 \mathrm{~N}$ & $200,0000 \mathrm{~N}$ & $10,0000 \mathrm{~N}$ & 10.000011 & 200,0000 \\
\hline 213184 & 2,0060 & 2.0000 & 0.5000 & 0.2000 & 200,0000 & $0.5000 \mathrm{~N}$ & $200.0000 \mathrm{~N}$ & $10,0000 \mathrm{H}$ & 20,0000 & 200,0000 \\
\hline 213185 & 2.0000 & 1.5000 & 0.7000 & 0.1500 & 500.0000 & $0.5000 \mathrm{~N}$ & $200,0000 \mathrm{~N}$ & 10.000011 & 20,0000 & 200,0000 \\
\hline
\end{tabular}


Table 1.--Analytical data for rock chip sampling--Continued

PRINTOUT FOR SPEC/AA

\begin{tabular}{|c|c|c|c|c|c|c|c|c|c|c|}
\hline SAMPLE & S-RE & $S-8 I$ & $s-C D$ & $s-c 0$ & S-CR & s-cu & $S-L A$ & $s-10$ & $5-N B$ & S-MI \\
\hline 213170 & $1.0000 \mathrm{~N}$ & $10.0000 \mathrm{~N}$ & $20.0000 \mathrm{~N}$ & $5.0000 \mathrm{~L}$ & 20.0000 & 10.0000 & $20.0000 \mathrm{~N}$ & & & \\
\hline 213171 & $1.0000 \mathrm{~N}$ & $10.0000 \mathrm{~N}$ & $20.0000 \mathrm{~N}$ & $5.0000 \mathrm{~K}$ & 30.0000 & 5.0000 & $20.0000 \mathrm{~N}$ & $5,0000 \mathrm{~N}$ & $20.0000 \mathrm{~N}$ & 15.0000 \\
\hline 213172 & $1.0000 \mathrm{~N}$ & $10.0000 \mathrm{~K}$ & $20.0000 \mathrm{~N}$ & 10.0000 & 50.0000 & 10.0000 & $20.0000 \mathrm{H}$ & $5.0000 \mathrm{~N}$ & $20.0000 \mathrm{~N}$ & 30.0000 \\
\hline $\begin{array}{l}213173 \\
213174\end{array}$ & $\begin{array}{l}1.0000 \mathrm{~N} \\
1.0000 \mathrm{~N}\end{array}$ & $\begin{array}{l}10.000011 \\
10.000011\end{array}$ & $\begin{array}{l}20.0000 \mathrm{~N} \\
20.0000 \mathrm{~N}\end{array}$ & $\begin{array}{l}20.0000 \\
10,0000\end{array}$ & $\begin{array}{l}100,0000 \\
100,0000\end{array}$ & $\begin{array}{r}10,0000 \\
7.0000\end{array}$ & $\begin{array}{l}20,0000 \mathrm{~N} \\
20,0000 \mathrm{~N}\end{array}$ & $\begin{array}{l}5.0000 \mathrm{H} \\
5.0000 \mathrm{~N}\end{array}$ & $\begin{array}{l}20.0000 \mathrm{~N} \\
20.0000 \mathrm{~N}\end{array}$ & $\begin{array}{l}50,0000 \\
30,0000\end{array}$ \\
\hline 213175 & $1.0000 \mathrm{H}$ & $10,0000 \mathrm{~N}$ & $20.0000 \mathrm{~N}$ & 5.0000 & 15.0000 & 5.0000 & 20.000011 & 5.00001 & $20.0000 \mathrm{H}$ & 15.0000 \\
\hline $\begin{array}{l}213176 \\
213177\end{array}$ & $\begin{array}{l}1.0000 \mathrm{H} \\
1.0000 \mathrm{~N}\end{array}$ & $\begin{array}{l}10.0000 \mathrm{~N} \\
10.0 \mathrm{CO0N}\end{array}$ & $\begin{array}{l}20.0000 \mathrm{~N} \\
20.0000 \mathrm{~N}\end{array}$ & $\begin{array}{r}15,0000 \\
7.0000\end{array}$ & $\begin{array}{l}70.0000 \\
20.0000\end{array}$ & $\begin{array}{l}15.0000 \\
10.0000\end{array}$ & $\begin{array}{l}20.0000 \mathrm{~N} \\
20.0000 \mathrm{~N}\end{array}$ & $\begin{array}{l}5.0000 \mathrm{H} \\
5.0000 \mathrm{H}\end{array}$ & $\begin{array}{l}20.0000 \mathrm{H} \\
20,0000 \mathrm{~N}\end{array}$ & $\begin{array}{r}50.0000 \\
7.0000\end{array}$ \\
\hline $\begin{array}{l}213178 \\
213179 \\
213180\end{array}$ & $\begin{array}{l}1.0000 \mathrm{~N} \\
1.0000 \mathrm{H} \\
1.0000 \mathrm{H}\end{array}$ & $\begin{array}{l}10.0000 \mathrm{H} \\
10.0000 \mathrm{H} \\
10.0000 \mathrm{H}\end{array}$ & $\begin{array}{l}20.0000 \mathrm{H} \\
20.000 \mathrm{HH} \\
20.0000 \mathrm{~N}\end{array}$ & $\begin{array}{l}5,0000 \mathrm{~N} \\
7,0000 \\
7,0000\end{array}$ & $\begin{array}{l}30.0000 \\
50,0000 \\
50.0000\end{array}$ & $\begin{array}{l}20.0000 \\
10.0000 \\
30,0000\end{array}$ & $\begin{array}{l}20.0000 \mathrm{~N} \\
20.0000 \mathrm{~N} \\
20.0000 \mathrm{~N}\end{array}$ & $\begin{array}{l}5.000 \mathrm{~N} \\
5.0000 \mathrm{~N} \\
5.0000 \mathrm{~N}\end{array}$ & $\begin{array}{l}20,6000 \mathrm{~N} \\
20,0000 \mathrm{H} \\
20,000 \mathrm{~N}\end{array}$ & $\begin{array}{r}7.0000 \\
20.0000 \\
20.0000\end{array}$ \\
\hline 213181 & $1.0000 \mathrm{~N}$ & $10.0000 \mathrm{~N}$ & $20.0000 \mathrm{~N}$ & $5.0000 \mathrm{H}$ & 30.0000 & 50.0000 & $20.0000 \mathrm{~N}$ & $5.0000 \mathrm{~N}$ & $20.0000 \mathrm{H}$ & 7,0000 \\
\hline 213182 & 1.00001 & $10.0000 \mathrm{~N}$ & $20.0000 \mathrm{~N}$ & 10.0000 & 50.0000 & 20.0000 & $20.0000 \mathrm{~N}$ & $5.0000 \mathrm{~N}$ & 20,000011 & 30.0000 \\
\hline 213183 & $1.0000 \mathrm{~L}$ & 10.000011 & $20.0000 \mathrm{~N}$ & 20.0000 & 15,0000 & 15,0000 & $20.0000 \mathrm{~N}$ & $5,0000 \mathrm{~N}$ & $20.0000 L$ & 15.0000 \\
\hline $\begin{array}{l}213184 \\
213185\end{array}$ & 1.00001 & $10.0000 \mathrm{~N}$ & $20,0000 \mathrm{~N}$ & $5,0000 \mathrm{~N}$ & 70.0000 & 15.0000 & $20.0000 \mathrm{~N}$ & $5.0000 \mathrm{~N}$ & $20.0000 \mathrm{~N}$ & 20.0000 \\
\hline SAMPLE & S-PB & $5-5 B$ & S-SC & S-SN & S-sk & $s-4$ & $s-w$ & $\underline{\mathbf{s}}-\gamma$ & $s-2 N$ & $S-2 R$ \\
\hline $\begin{array}{l}213170 \\
213171\end{array}$ & $\begin{array}{l}10.0000 L \\
10.000011\end{array}$ & $\begin{array}{l}100.0000 \mathrm{~N} \\
100.0000 \mathrm{~N}\end{array}$ & $\begin{array}{l}5,0000 \\
5,0000 \mathrm{~L}\end{array}$ & $\begin{array}{l}10.0000 \mathrm{~N} \\
10.0000 \mathrm{~N}\end{array}$ & $\begin{array}{r}150.0000 \\
1000.0000\end{array}$ & $\begin{array}{l}70.0000 \\
20.0000\end{array}$ & $\begin{array}{l}50.0000 \mathrm{~N} \\
50.0000 \mathrm{~N}\end{array}$ & $\begin{array}{l}10.0000 \\
10.0000\end{array}$ & $\begin{array}{l}200.0000 \mathrm{H} \\
200.0000 \mathrm{H}\end{array}$ & $\begin{array}{l}70.0000 \\
10.00006\end{array}$ \\
\hline 213172 & 10.00001 & $100.0000 \mathrm{~N}$ & 5,0000 & $10,0000 \mathrm{~N}$ & 100,0000 & 50.0000 & 50,000014 & $10.0000 \mathrm{~L}$ & $200.0000 \mathrm{~N}$ & 100.0000 \\
\hline 213173 & $10.0000 \mathrm{~L}$ & $100.0000 \mathrm{~N}$ & 7.0000 & $10.0000 \mathrm{~N}$ & 200.0000 & 70.0000 & $50,0000 \mathrm{~N}$ & $10.0000 \mathrm{~L}$ & 200.000011 & 100.0000 \\
\hline 213174 & 10.00002 & $100,0006 \mathrm{~N}$ & 7.0000 & $10.0000 \mathrm{~N}$ & 200.0000 & 50.0000 & 50,000011 & $10,0000 \mathrm{~L}$ & $200.0000 \mathrm{~N}$ & 100,0000 \\
\hline 213175 & $10.000 \mathrm{LL}$ & $100.0000 \mathrm{~N}$ & 7.0000 & $10,0000 \mathrm{~N}$ & 300,0000 & 50,0000 & $50,0000 \mathrm{~N}$ & 10.0000 & $200.0000 \mathrm{H}$ & 150.0000 \\
\hline 213176 & $10.0000 \mathrm{~L}$ & $100,0000 \mathrm{~N}$ & 7.0000 & $10.0000 \mathrm{~N}$ & 500.0000 & 70.0000 & $50,0000 \mathrm{~N}$ & 10,00001 & $200.0000 \mathrm{~N}$ & 100.0000 \\
\hline 213177 & 10.00001 & $100.0000 \mathrm{H}$ & 10.0000 & $10.0000 \mathrm{~N}$ & 300.0000 & 70.0000 & $50,0000 \mathrm{~N}$ & 10,0000 & $200.0000 \mathrm{~N}$ & 70.0000 \\
\hline 213178 & 20.0000 & $100.0000 \mathrm{H}$ & 5.0000 & $10.0000 \mathrm{~N}$ & 100,0000 & 50.0000 & $50,0000 \mathrm{H}$ & $10,0000 \mathrm{~L}$ & $200.0000 \mathrm{~N}$ & 70,0000 \\
\hline $\begin{array}{l}213179 \\
213180\end{array}$ & $\begin{array}{l}10.000011 \\
10.0000 \mathrm{~N}\end{array}$ & $\begin{array}{l}100.0000 \mathrm{H} \\
100.0000 \mathrm{~N}\end{array}$ & $\begin{array}{l}10,0000 \\
10,0000\end{array}$ & $\begin{array}{l}10.0000 \mathrm{~N} \\
10.0000 \mathrm{~N}\end{array}$ & $\begin{array}{l}100,0000 \\
200,0000\end{array}$ & $\begin{array}{l}50,0000 \\
50,0000\end{array}$ & $\begin{array}{l}50,0000 \mathrm{~N} \\
50,0000 \mathrm{~N}\end{array}$ & $\begin{array}{l}10.0000 \\
10.0000\end{array}$ & $\begin{array}{l}200.0000 \mathrm{~N} \\
200.0000 \mathrm{H}\end{array}$ & $\begin{array}{l}100.0000 \\
100.0000\end{array}$ \\
\hline 213181 & $10.0000 \mathrm{~L}$ & $100.0000 \mathrm{~N}$ & 7,0000 & $10.0000 \mathrm{~N}$ & 300.0000 & 50.0000 & $50.0000 \mathrm{~N}$ & 10.0000 & $200.0000 \mathrm{~N}$ & 100,0000 \\
\hline 213182 & $10.0000 \mathrm{~N}$ & $100.0000 \mathrm{~N}$ & 15.0000 & $10,0000 \mathrm{H}$ & $100,0000 \mathrm{~N}$ & 100.0000 & 50.000004 & 20.0000 & $200.0000 \mathrm{~N}$ & 150.0000 \\
\hline 213183 & 10.000011 & $100.0000 \mathrm{~N}$ & 15,0000 & $10.0000 \mathrm{~N}$ & 500.0000 & 100,0000 & 50.00004 & 30.0000 & $200.0000 \mathrm{~N}$ & 150,0000 \\
\hline 213184 & $10.0000 \mathrm{~N}$ & $100.0000 \mathrm{H}$ & 10.0000 & $10.0000 \mathrm{~N}$ & $100,0000 \mathrm{~N}$ & 70,0000 & $50.0000 \mathrm{~N}$ & 10.0000 & $200.0000 \mathrm{~N}$ & 100,0000 \\
\hline 213185 & $10.0000 \mathrm{~L}$ & $100.0000 \mathrm{~K}$ & 7.0000 & $10,0000 \mathrm{~N}$ & 500,0000 & 50.0000 & $50,0000 \mathrm{~N}$ & 10.0000 & $200.0000 \mathrm{~N}$ & 100.0000 \\
\hline
\end{tabular}


Table 1.--Analytical data for rock chip sampling--Continued

PRINTOUT FOR SPEC/AA

$\begin{array}{lll}\text { SANPLE } & \text { AA-AU-P } & A Z-A G \\ & & \\ 213170 & 0.0500 \mathrm{~N} & 0.00000 \mathrm{~B} \\ 213171 & 0.0500 \mathrm{~N} & 0.0000 \mathrm{~B} \\ 213172 & 0.1000 & 0.0000 \mathrm{~B} \\ 213173 & 0.0500 \mathrm{~N} & 0.0000 \mathrm{~B} \\ 213174 & 0.0500 \mathrm{~N} & 0.0000 \mathrm{~B} \\ 213175 & 0.0500 \mathrm{~N} & 0.0000 \mathrm{~B} \\ 21317 \mathrm{~S} & 0.0500 \mathrm{~N} & 0.0000 \mathrm{~B} \\ 213177 & 0.0500 \mathrm{~N} & 0.0000 \mathrm{~B} \\ 213178 & 0.3800 & 0.0000 \mathrm{~B} \\ 213179 & 0.0500 \mathrm{~N} & 0.0000 \mathrm{~B} \\ 213180 & 0.0600 & 0.0000 \mathrm{~B} \\ 213181 & 0.1000 & 0.0000 \mathrm{~B} \\ 213182 & 0.0500 & 0.0000 \mathrm{~B} \\ 213183 & 0.0500 \mathrm{H} & 0.0000 \mathrm{~B} \\ 213184 & 0.1600 & 0.0000 \mathrm{~B} \\ 213185 & 0.0500 \mathrm{~N} & 0.0000 \mathrm{~B}\end{array}$

\begin{tabular}{|c|c|c|c|c|c|c|c|c|c|c|}
\hline SAMFLE & S-FE & S-146 & S-CA & S-II & S-HiN & S-AB & S-AS & S-AU & S-8 & $S-B A$ \\
\hline $\begin{array}{l}213185 \\
213186\end{array}$ & $\begin{array}{l}2.0000 \\
3.0000\end{array}$ & $\begin{array}{l}1.5000 \\
3.0000\end{array}$ & $\begin{array}{l}0.7000 \\
1.5000\end{array}$ & $\begin{array}{l}0.1500 \\
0.2000\end{array}$ & $\begin{array}{l}500.0000 \\
700.0000\end{array}$ & $\begin{array}{l}0.5000 \mathrm{~N} \\
0.5000 \mathrm{~N}\end{array}$ & $\begin{array}{l}200.0000 \mathrm{H} \\
200.0000 \mathrm{H}\end{array}$ & $\begin{array}{l}10.0000 \mathrm{~N} \\
10.0000 \mathrm{~N}\end{array}$ & $\begin{array}{l}20.0000 \\
10.0000\end{array}$ & $\begin{array}{l}200.0000 \\
300.0000\end{array}$ \\
\hline 213187 & 2.0000 & $3: 0000$ & 1.0000 & 0.2000 & 500.0000 & $0.5000 \mathrm{~N}$ & $200.0000 \mathrm{~N}$ & $10.0000 \mathrm{~N}$ & 10.0000 & 200.0000 \\
\hline 213188 & 3.0000 & 5.0000 & 1.5000 & 0.3000 & 1000,0000 & $0.5000 \mathrm{~N}$ & $200,0000 \mathrm{~N}$ & $10,0000 \mathrm{~N}$ & 20.0000 & 150.0000 \\
\hline SAPLE & S-BE & $S-B I$ & $s-c a$ & $s-c 0$ & $5-C B$ & $s-c u$ & $S-L A$ & $s-130$ & $S-A B$ & $S-N !$ \\
\hline $\begin{array}{l}213185 \\
213185\end{array}$ & $\begin{array}{l}1.0000 \mathrm{~N} \\
1.0000 \mathrm{~N}\end{array}$ & $\begin{array}{l}10.0000 \mathrm{~N} \\
10.0000 \mathrm{~N}\end{array}$ & $\begin{array}{l}20.0000 \mathrm{NH} \\
20.0000 \mathrm{~N}\end{array}$ & $\begin{array}{c}5.00000 \\
15.0000\end{array}$ & $\begin{array}{l}20.0000 \\
20.0000\end{array}$ & $\begin{array}{c}5.0000 \mathrm{~L} \\
10.0000\end{array}$ & $\begin{array}{l}20.0000 \mathrm{~N} \\
20.0000 \mathrm{~N}\end{array}$ & $\begin{array}{l}5.0000 \mathrm{~N} \\
5.0000 \mathrm{~N}\end{array}$ & $\begin{array}{l}20.0000 \mathrm{~N} \\
20.0000 \mathrm{~N}\end{array}$ & $\begin{array}{l}5.00001 \\
5.0000\end{array}$ \\
\hline $\begin{array}{l}213187 \\
213188\end{array}$ & $\begin{array}{l}1.0000 \mathrm{~N} \\
1.0000 \mathrm{H}\end{array}$ & $\begin{array}{l}10,0000 \mathrm{~N} \\
10,0000 \mathrm{H}\end{array}$ & $\begin{array}{l}20,0000 \mathrm{~N} \\
20,0000 \mathrm{H}\end{array}$ & $\begin{array}{c}5.0000 \mathrm{~N} \\
20.0000\end{array}$ & $\begin{array}{r}20.0000 \\
100,0000\end{array}$ & $\begin{array}{l}15,0000 \\
15,0000\end{array}$ & $\begin{array}{l}20.0000 \mathrm{~N} \\
20.0000 \mathrm{~N}\end{array}$ & $\begin{array}{l}5,0000 \mathrm{~N} \\
5,0000 \mathrm{~N}\end{array}$ & $\begin{array}{l}20,0000 \mathrm{~K} \\
20,0000 \mathrm{~N}\end{array}$ & $\begin{array}{r}7,0000 \\
50,0000\end{array}$ \\
\hline SAKPLE & S-PB & $5-58$ & $5-5 C$ & S-SN & S-SR & $s-v$ & $s-4$ & S-Y & $S-2 M$ & $5-2 R$ \\
\hline $\begin{array}{l}213185 \\
213186\end{array}$ & $\begin{array}{l}10.0000 \mathrm{~L} \\
10.0000 \mathrm{~N}\end{array}$ & $\begin{array}{l}100.0000 \mathrm{~N} \\
100.0000 \mathrm{~N}\end{array}$ & $\begin{array}{r}7,0000 \\
15,0000\end{array}$ & $\begin{array}{l}10.0000 \mathrm{~N} \\
10.0000 \mathrm{~N}\end{array}$ & $\begin{array}{l}500.0000 \\
500.0000\end{array}$ & $\begin{array}{l}50.0000 \\
70.0000\end{array}$ & $\begin{array}{l}50.0000 \mathrm{H} \\
50.0000 \mathrm{~N}\end{array}$ & $\begin{array}{l}10.0000 \\
10.0000\end{array}$ & $\begin{array}{l}200.0000 \mathrm{~N} \\
200.0000 \mathrm{H}\end{array}$ & $\begin{array}{r}100.0000 \\
70.0000\end{array}$ \\
\hline 3187 & $10.0000 \mathrm{~N}$ & $100.0000 \mathrm{~N}$ & 10.0000 & $10.0000 \mathrm{~N}$ & 300,0000 & 50.0000 & $50.0000 \mathrm{~N}$ & 15.0000 & $200,0000 \mathrm{~N}$ & 100,0000 \\
\hline 213188 & $10.0000 \mathrm{H}$ & $100.0000 \mathrm{~N}$ & 15.0000 & $10.0000 \mathrm{~N}$ & 500.0000 & 100.0000 & $50.0000 \mathrm{~N}$ & 20.0000 & $200.0000 \mathrm{H}$ & 150.0000 \\
\hline
\end{tabular}

$\begin{array}{lll}\text { SAIPLE } & \text { AA-AU-P } & \text { AZ-AG } \\ & & \\ 213185 & 0.0500 \mathrm{~N} & 0.0000 \mathrm{~B} \\ 213186 & 0.0500 & 0.0000 \mathrm{~B} \\ 213187 & 0.0500 \mathrm{~N} & 0.0000 \mathrm{~B} \\ 213188 & 0.0500 \mathrm{~N} & 0.0000 \mathrm{~B}\end{array}$


Table 2-Atomic-absorption analytical data for trench sampling in the Meshaheed area [Leaders indicate none detected; $<=$ less than, $>=$ greater than value indicated. Values in parts per million]

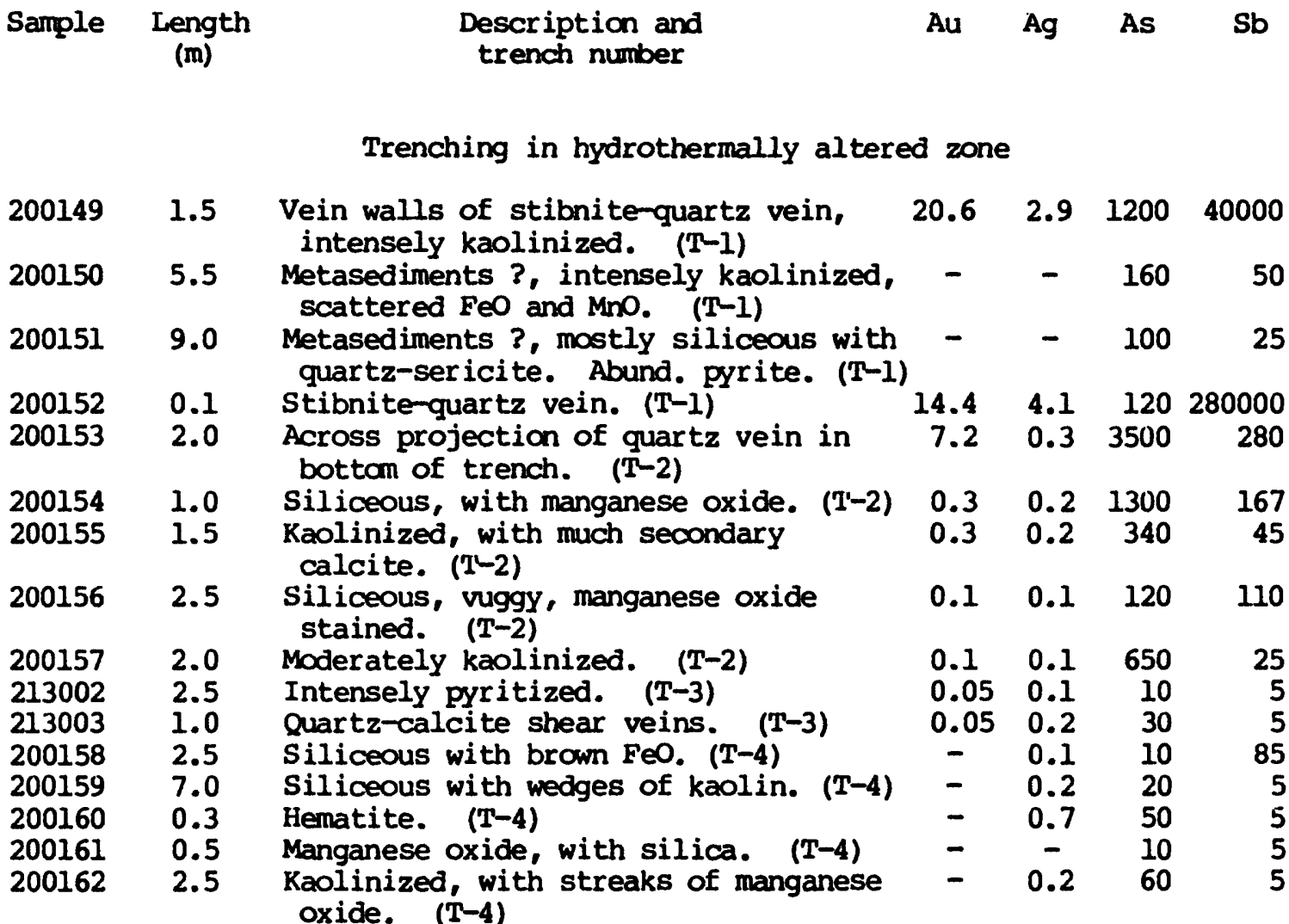

Trenching in Pluton Southeast ancient workings

$\begin{array}{lllllrr}200163 & 6.0 & \text { Metasediments, black, indurated. } & - & - & 10 & 5 \\ 200164 & 0.1 & \text { Fault gouge. } & 0.1 & - & 1000 & 49 \\ 200165 & 0.05 & \text { Fault gouge. } & 1.4 & - & 800 & 550\end{array}$

Rock chip sampling in and around small ancient workings in basalt

200980

200981

200982

200983

200984

200985

200986

200987

200988
Dark gray quartz pod adjacent to workings.

Quartz-calcite veinlets in basalt. Across back of small workings-fractured - basalt with quartz-calcite.

Across $50 \mathrm{~cm}$ of quartz veinlets. $\quad .05-700$

Quartz, black, with calcite, $15 \mathrm{~cm}$.

Quartz, in portal of workings.

Basalt with pyrrhotite.

Geophysical ancmaly east of Meshaheed

Hematitic gossan, dense, flinty. - 0.2

Gossan, dark brown, dense. $\quad-0.9$ 


\section{DATA STORAGE}

Petrographic descriptions, sample locations, thin sections, and results of chemical analyses are stored in Data-file USGS-DF-04-09 (Smith, 1984) in the Jeddah of fice of the U. S. Geological Survey Saudi Arabian Mission.

Data on mineral occurrences in the Meshaheed area have been updated for the following MODS number:

1266 Meshaheed Au updated 11/84

\section{REFERENCES}

Allen, M. S, Tidball, R. R., and Samater, R. M., 1984, Interpretation of geochemical data from panned concentrates of wadi sediments using $R$-Mode factor analysis, Jabal Habashi quadrangle, sheet 26F, Kingdom of Saudi Arabia: Saudi Arabian Deputy Ministry for Mineral Resources Open-File Report USGS-OF-04-45, 51 p. Also, 1985, U.S. Geologica1 Survey Open-File Report 85-5.

Bagdady, A. Y., Whitlow, J. W., and Roberts, R. J., 1978, Placer gold deposits in the Mahd adh Dhahab district, Kingdom of Saudi Arabia: U. S. Geological Survey Open-File Report 78=9074, 38 p.

Cole, J. C., inpree, Geology of the Aban al Ahmar quadrangle, sheet 25F, Kingdom of Saudi Arabia: Sardi Arabian Deputy Ministry for Miraz 2 Resources Geoscience map GM-105A.

Cole, J. C. and Hedge, C. E., 1985, Late Proterozoic geochronology in the northeastern Shield of Saudi Arabia: Unpublished data on file in the Jeddah of fice of the U.S. Geological Survey Saudi Arabian mission.

Flanigan, V. J., and Zablocki, C. J., 1984, An evaluation of the applicability of the telluric-electric and audio- magnetotelluric methods to mineral assessment on the Arabian Shield, Kingdom of Saudi Arabia: Saudi Arabian Deputy Ministry for Mineral Resources Open File Report USGS-OF-04-26, 61 p. Also, U.S. Geological Survey Open-File Report 84-425.

Mutschler, F. E., Wright, E. G., Ludington, S., and Abbot, J. T., 1981, Granite molybdenite systems, Economic Geology, volume 76 , No. 4, pp. 874-897.

Schmidt, D. L., Puffet, W. P., Campbell, W. L., and Al-Koulak, Z. H., 1981, Gold placer and Quaternary stratigraphy of the Jabal Mokhyat area, southern Najd Province, Kingdom of Saudi Arabia: U. S. Geological Survey Open-File Report $82-414,69 \mathrm{p}$.

Shannon, J. R., Walker, M. B., Carten, R. B., and Geraghty, E. P., 1982, Unidirectional solidification textures and their significance in determining relative ages of intrusions at the Henderson Mine, Colorado, Geology, volume 10, pp. 293-297.

Smith, C. W., and Samater, R. M., 1984, Preliminary report on the gold deposits at Meshaheed, Kingdom of Saudi Arabia: Saudi Arabian Deputy Ministry for Mineral Resources Open-File Report USGS-OF-04-29, 38 p. Also, 1985, U.S.

Geological Survey Open-File Report 85-9. 
Smith, C. W., Samater, R. M., Hussain, M. A., Basheer, M. A., and Trent, V. A., 1984, Preliminary report on gold deposits of the An Najadi-Wuday region, Samirah and Uqlat as Suqur quadrangles, Kingdom of Saudi Arabia: Saudi Arabian Deputy Ministry for Mineral Resources Open-File Report USGS-OF-04-48, 26 p. Also, U.S. Geological Survey Open-File Report 85-129.

White, W. H., Bookstrom, A. A., Kamilli, R. J., Ganster, M. W., Smith, R. P., Ranta, D. E., and Steininger, R. C., 1981, Character and origin of Climax-type molybdenum deposits: Economic Geology, 75th anniversary volume, pp. 270-316.

Williams, P. L., 1984, Reconnaissance geology of the Samirah Quadrangle, Sheet 26/42 C, Kingdom of Saudi Arabia: Saudi Arabian Deputy Ministry for Mineral Resources Open-File Report USGS-OF-04-3, 33 p. Also, 1984, U.S. Geological Survey Open-File Report 84-383. 